\title{
ChaMP Serendipitous Galaxy Cluster Survey
}

\section{Citation}

Barkhouse, W. A., P. J. Green, A. Vikhlinin, D.\#W. Kim, D. Perley, R. Cameron, J. Silverman, et al. 2006. "ChaMP Serendipitous Galaxy Cluster Survey." The Astrophysical Journal 645 (2) (July 10): 955-976. doi:10.1086/504457.

\section{Published Version}

doi:10.1086/504457

\section{Permanent link}

http://nrs.harvard.edu/urn-3:HUL.InstRepos:30212142

\section{Terms of Use}

This article was downloaded from Harvard University's DASH repository, and is made available under the terms and conditions applicable to Other Posted Material, as set forth at http:// nrs.harvard.edu/urn-3:HUL.InstRepos:dash.current.terms-of-use\#LAA

\section{Share Your Story}

The Harvard community has made this article openly available.

Please share how this access benefits you. Submit a story.

Accessibility 
The Astrophysical Journal, 645:955-976, 2006 July 10

(C) 2006. The American Astronomical Society. All rights reserved. Printed in U.S.A.

\title{
ChaMP SERENDIPITOUS GALAXY CLUSTER SURVEY
}

\author{
W. A. Barkhouse, ${ }^{1,2}$ P. J. Green, ${ }^{1,2}$ A. Vikhlinin, ${ }^{2}$ D.-W. Kim, ${ }^{2}$ D. Perley, ${ }^{3}$ R. Cameron, ${ }^{4}$ J. Silverman, $^{1,5}$ A. Mossman, ${ }^{2}$ \\ R. Burenin, ${ }^{6}$ B. T. Jannuzi, ${ }^{7}$ M. Kim, ${ }^{2}$ M. G. Smith, ${ }^{8}$ R. C. Smith, ${ }^{8}$ H. Tananbaum, ${ }^{2}$ and B. J. Wilkes ${ }^{2}$ \\ Received 2005 June 21; accepted 2006 March 19
}

\begin{abstract}
We present a survey of serendipitous extended X-ray sources and optical cluster candidates from the Chandra Multiwavelength Project (ChaMP). Our main goal is to make an unbiased comparison of X-ray and optical cluster detection methods. In 130 archival Chandra pointings covering $13 \mathrm{deg}^{2}$, we use a wavelet decomposition technique to detect 55 extended sources, of which 6 are nearby single galaxies. Our X-ray cluster catalog reaches a typical flux limit of about $\sim 10^{-14} \mathrm{ergs} \mathrm{cm}^{-2} \mathrm{~s}^{-1}$, with a median cluster core radius of $21^{\prime \prime}$. For 56 of the $130 \mathrm{X}$-ray fields, we use the ChaMP's deep NOAO $4 \mathrm{~m}$ MOSAIC $g^{\prime}, r^{\prime}$, and $i^{\prime}$ imaging to independently detect cluster candidates using a Voronoi tessellation and percolation (VTP) method. Red-sequence filtering decreases the galaxy fore- and background contamination and provides photometric redshifts to $z \sim 0.7$. From the overlapping 6.1 deg ${ }^{2} \mathrm{X}-\mathrm{ray} / \mathrm{optical}$ imaging, we find 115 optical clusters (of which $11 \%$ are in the X-ray catalog) and $28 \mathrm{X}$-ray clusters (of which $46 \%$ are in the optical VTP catalog). The median redshift of the 13 X-ray/optical clusters is 0.41 , and their median X-ray luminosity $(0.5-2 \mathrm{keV})$ is $L_{\mathrm{X}}=(2.65 \pm 0.19) \times 10^{43} \mathrm{ergs} \mathrm{s}^{-1}$. The clusters in our sample that are only detected in our optical data are poorer on average $(\sim 4 \sigma)$ than the X-ray/optically matched clusters, which may partially explain the difference in the detection fractions.
\end{abstract}

Subject headings: galaxies: clusters: general — surveys — X-rays: galaxies: clusters

\section{INTRODUCTION}

A primary goal of modern astronomy is to study the formation and evolution of galaxies. Clusters of galaxies provide us with laboratories in which galaxy evolution can be studied over a large range in cosmic look-back time. The high-density cluster environment probes the impact of high galaxy density on the fate of the cluster galaxy population. Interactions, mergers, and dynamical effects (e.g., tidal forces and ram pressure stripping) may play significant roles in shaping galaxy evolution in these type of locales (e.g., Dubinski 1998; Moore et al. 1999).

Galaxy clusters are also the most massive, mainly virialized, concentrations of matter in the universe and act as tracers of the underlying dark matter. Clusters thus also play a key role constraining fundamental cosmological parameters such as $\Omega_{m}$ (the matter-density parameter) and $\sigma_{8}$ (the rms density fluctuation on a scale of $8 h^{-1} \mathrm{Mpc}$ ). The number density of clusters as a function of mass and redshift strongly depends on $\Omega_{m}$ and $\sigma_{8}$ (see Rosati et al. 2002 and references therein). This remarkable fea-

\footnotetext{
1 Visiting Astronomer, Kitt Peak National Observatory (KPNO) and Cerro Tololo Inter-American Observatory (CTIO), National Optical Astronomy Observatory (NOAO), operated by the Association for Universities for Research in Astronomy (AURA), Inc., under contract to the National Science Foundation (NSF).

${ }^{2}$ Harvard-Smithsonian, Center for Astrophysics, 60 Garden Street, Cambridge, MA 02138.

3 Department of Astronomy, 601 Campbell Hall, University of California, Berkeley, CA 94720.

4 Stanford Linear Accelerator Center, 2575 Sand Hill Road, Menlo Park, CA 94025 .

5 Max-Plank-Institut für extraterrestrische Physik, Giessenbachstrasse, 85741 Garching, Germany.

6 Space Research Institute, Russian Academy of Sciences, Profsoyuznaya Street 84/32, Moscow 117997, Russia.

7 National Optical Astronomy Observatory, P.O. Box 26732, Tucson, AZ 85726.

${ }^{8}$ Cerro Tololo Inter-American Observatory, National Optical Astronomy Observatory, Casilla 603, La Serena, Chile.
}

ture of hierarchical cluster formation, via the Press-Schechter formalism (Press \& Schechter 1974), affords the opportunity to provide an independent confirmation of various cosmological quantities obtained recently by other techniques (e.g., the Wilkinson Microwave Anisotropy Probe [WMAP]; Bennett et al. 2003).

To facilitate the investigation of galaxy cluster evolution and provide constraints on cosmological parameters, a large sample of galaxy clusters spanning a redshift range from $0<z<1.5$ is required. The search for galaxy clusters has been conducted mainly using optical and X-ray techniques. Although these methods overlap in their ability to distinguish galaxy clusters from the general background, they sample different regions of parameter space that encompass nearly the complete range in physical attributes of clusters. For example, X-ray techniques detect clusters via extended emission from the hot gas that makes up the intracluster medium (ICM; e.g., Vikhlinin et al. 1998b). X-ray detection suffers little from source confusion (e.g., Basilakos et al. 2004; Popesso et al. 2004) but tends to select more massive, virialized clusters. By contrast, optical methods rely on the detection of an overdensity of galaxies or a population of early-type galaxies with a narrow range of colors (red sequence; Gladders \& Yee 2000). Optical methods are generally more sensitive to nonvirialized (or young) systems than X-ray searches, but they are also susceptible to projection effects and bias toward more evolved galaxy populations. Multiwavelength cluster detection schemes help to ensure a higher degree of completeness and reliability in any cluster compilation (Postman 2002).

Several recent studies have compared cluster samples compiled from independent techniques using optical and X-ray data. Donahue et al. (2002), for example, applied a matched-filter method to optical data and found that $60 \%$ (26 out of 43) of ROSAT X-ray clusters had optical matches. They also determined that optical clusters/groups outnumbered X-ray extended sources by a factor of 3. Gilbank et al. (2004), applying a matched-filter algorithm to optical data, found $75 \%$ matches $(9 / 12)$ to a sample 
of ROSAT X-ray clusters. Using a cluster detection method based on the red sequence of early-type cluster galaxies, Gilbank et al. (2004) was able to achieve a matched fraction of $100 \%(10 / 10)$ using the same X-ray data set. In addition, Basilakos et al. (2004) and Kolokotronis et al. (2006) — using a smoothing percolation technique on optical data-found matched fractions of $75 \%(3 / 4)$ and $68 \%(13 / 19)$, respectively, for extended X-ray sources compiled from archival XMM-Newton observations. In all such studies, the matched fractions depend on the relative optical/X-ray flux limits and the sensitivity of the detection algorithms.

The aims of this paper are to present a new serendipitous X-ray cluster sample based on data from the Chandra Multiwavelength Project (ChaMP; Kim et al. 2004a; Green et al. 2004) ${ }^{9}$ and to make an unbiased comparison of X-ray and optical cluster detection methods. We thus explore a variety of questions: Are there massive, X-ray-luminous clusters that are optically poor? Do all massive clusters emit X-rays? What types of optical clusters retain hot gas, and why? In addition, we present the X-ray and optical properties of our sample of serendipitously detected clusters/ groups, including a comparison of X-ray luminosity with optical cluster richness. Finally, we provide the community with a compilation of newly discovered clusters/groups that can be used in conjunction with other samples to constrain cosmological parameters. This sample should also help to address how the more numerous (but less well studied) low-luminosity clusters and groups evolve.

This paper is organized as follows. In $\S 2$ we describe the sample selection and X-ray and optical observations. In $\S 3$ we discuss our X-ray and optical cluster detection methods. The properties of our X-ray and optical cluster candidates are presented in $\S 4$, along with a comparison of the two compilations. Finally, in $\S 5$ we compare our results with previous studies and discuss possible bias inherit in our X-ray and optical cluster detection schemes. Unless otherwise indicated, we use $\Omega_{m}=0.3$, $\Omega_{\lambda}=0.7$, and $H_{0}=70 \mathrm{~km} \mathrm{~s}^{-1} \mathrm{Mpc}^{-1}$ throughout.

\section{SAMPLE SELECTION AND OBSERVATIONS}

This study makes use of the data provided by ChaMP. ChaMP is a $\sim 13 \mathrm{deg}^{2}$ (based on Cycles 1 and 2 Chandra archival data) survey of serendipitous Chandra X-ray sources at flux levels $\left(f_{\mathrm{X}} \sim 10^{-15}\right.$ to $10^{-14} \mathrm{ergs} \mathrm{s}^{-1} \mathrm{~cm}^{-2}$ ), intermediate between the Chandra deep surveys and previous X-ray missions. Optical follow-up of ChaMP fields was conducted using the MOSAIC camera on the KPNO and CTIO $4 \mathrm{~m}$ telescopes. The mosaic imaging of ChaMP was designed to search for optical counterparts to active galactic nuclei (AGNs) in part to drive our spectroscopy identification program. At present, 56 mosaic fields in $g^{\prime}, r^{\prime}$, and $i^{\prime}$ to $r^{\prime} \lesssim 25$ (5 $\sigma$ detection) have been acquired (W. A. Barkhouse et al. 2006, in preparation). For a description of ChaMP methods, analysis, and early science results, see Kim et al. (2004a, 2004b, 2006), Green et al. (2004), Silverman (2004), and Silverman et al. (2005a, 2005b).

The X-ray data for this study are drawn from 130 fields selected from Chandra $\mathrm{AO} 1$ and $\mathrm{AO} 2$ observing periods. The fields were selected based on the following criteria: (1) include only ACIS imaging fields (excluding the ACIS-S4 chip); (2) include only fields more than $20^{\circ}$ from the Galactic plane to minimize extinction; (3) exclude fields dominated by large extended sources; (4) include no planetary observations; (5) include no survey observations by PI; and (6) include no fields close to the LMC, SMC, and M31 (see Kim et al. [2004a] for a detailed discussion of selection criteria and X-ray data reductions). Galactic $N_{\mathrm{H}}$ values are

\footnotetext{
9 See http://hea-www.harvard.edu/CHAMP.
}

taken from Stark et al. (1992) and are tabulated in Table 1 for fields containing at least one extended X-ray source.

The optical data for this study consists of ChaMP mosaic images acquired from NOAO $4 \mathrm{~m}$ telescopes in the $g^{\prime}, r^{\prime}$, and $i^{\prime}$ bandpasses (see Table 2). The optical and X-ray imaging overlap by $6.1 \mathrm{deg}^{2}$. These data are used for source identification and to compare optical cluster detection methods against X-ray techniques for the area in common (see $\S 3$ ). Details of image reduction and analysis for the initial sample of six ChaMP mosaic fields are presented in Green et al. (2004), and an overview of our complete sample of 56 fields is in W. A. Barkhouse et al. (2006, in preparation). In summary, our optical exposure times were scaled to the X-ray exposures to probe a constant X-ray/optical flux ratio. The optical follow-up was optimized to probe AGN counterparts and not faint galaxies at a similar redshift for a given $\mathrm{X}$-ray luminosity. The image reduction was performed using the mscred package within the IRAF ${ }^{10}$ environment. Object detection and photometry was conducted using SExtractor (Bertin \& Arnouts 1996). Photometric calibrations were done using standard stars from Landolt (1992), which were converted to the SDSS photometric system using the transformation equations from Fukugita et al. (1996). Table 2 summarizes the optical properties of the 36 mosaic fields that overlap X-ray fields containing X-ray-detected extended sources or optical cluster candidates.

\section{GALAXY CLUSTER DETECTION METHODS}

We have used the X-ray and optical data sets from ChaMP to search for and contrast galaxy cluster samples compiled independently from X-ray and optical cluster search techniques for the overlapping $6.1 \mathrm{deg}^{2}$ sky coverage. In the following sections we describe each detection method, with an emphasis on the description of the optical technique (see Vikhlinin et al. 1998b for a detailed description of the extended X-ray source detection algorithm).

\subsection{Extended X-Ray Source Detection}

The extended X-ray source detection is based on a wavelet decomposition technique - plus a maximum likelihood method to determine the significance of each detected extended sourcethat is similar to the method described in Vikhlinin et al. (1998b). In brief, each extended source was detected in the $0.7-2 \mathrm{keV}$ energy band to maximize the contrast of the cluster ICM against the X-ray background. A Gaussian kernel was fit to each wavelet source and its best-fit radius was compared with the point-spread function (PSF) size appropriate for the measured off-axis angle. Those objects determined to be "pointlike" were then subtracted, and the detection process applied to the resultant image. The sample of X-ray sources deemed "extended" was then fit on the original image to a standard $\beta$ model, $I\left(r, r_{c}\right)=I_{0}[1+$ $\left.\left(r / r_{c}\right)^{2}\right]^{-3 \beta+0.5}$ (Cavaliere \& Fusco-Femiano 1976), with point sources masked out. Since a free fit was not possible due to the small number of photons expected for most sources, we fixed the value for $\beta$ at 0.67 (e.g., Vikhlinin et al. 1998b; Donahue et al. 2002; Moretti et al. 2004).

\subsubsection{Final X-Ray-Selected Cluster Catalog}

The initial sample of extended X-ray sources is comprised of PI target clusters, serendipitous clusters, nearby bright galaxies, and spurious detections caused by chip gaps, edge effects, etc. Visual inspection and cross-correlation to Chandra PI targets

\footnotetext{
${ }^{10}$ IRAF is distributed by NOAO, which is operated by AURA, Inc., under
} the cooperative agreement with the NSF 
TABLE 1

X-Ray-detected Extended Sources

\begin{tabular}{|c|c|c|c|c|c|}
\hline $\begin{array}{l}\text { Source Name } \\
\text { CXOMP }\end{array}$ & ObsID & $\begin{array}{c}\text { R.A. } \\
(\mathrm{J} 2000.0)\end{array}$ & $\begin{array}{c}\text { Decl. } \\
(\mathrm{J} 2000.0)\end{array}$ & $\begin{array}{c}\text { Exposure }^{\mathrm{a}} \\
\text { (s) }\end{array}$ & $\begin{array}{l}\text { Galactic } N_{\mathrm{H}}^{\mathrm{b}} \\
\left(10^{20} \mathrm{~cm}^{-2}\right)\end{array}$ \\
\hline J002650.2+171935 & 929 & 002650.2 & +171935.7 & 40,346 & 4.19 \\
\hline J005848.1-280035 ........................... & 2248 & 005848.1 & -280035.7 & 12,138 & 1.55 \\
\hline J010214.1+314915 ......................... & 521 & $\begin{array}{lll}01 & 0214.1\end{array}$ & +314915.6 & 54,166 & 5.50 \\
\hline $\mathrm{J} 010607.0+004943 \ldots \ldots \ldots \ldots \ldots \ldots \ldots . .$. & 2180 & 010607.0 & +004943.7 & 3757 & 3.15 \\
\hline 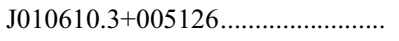 & 2180 & 010610.3 & +005126.1 & 3757 & 3.15 \\
\hline 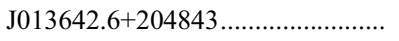 & 2129 & 013642.6 & +204843.7 & 45,094 & 5.71 \\
\hline 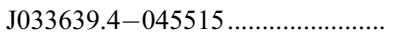 & 796 & 033639.4 & -045515.4 & 60,512 & 4.98 \\
\hline J033722.6-045906 ....................... & 796 & 033722.7 & -045905.8 & 60,512 & 4.98 \\
\hline J033755.1-050733 ………........... & 796 & 033755.1 & -050733.6 & 60,512 & 4.98 \\
\hline $\mathrm{J} 033757.8-050001 \ldots \ldots \ldots \ldots \ldots \ldots . .$. & 796 & 033757.8 & -050000.9 & 60,512 & 4.98 \\
\hline 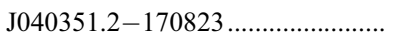 & 2182 & 040351.2 & -170823.2 & 3891 & 2.30 \\
\hline 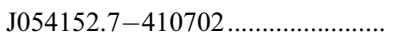 & 914 & 054152.7 & -410702.7 & 51,050 & 3.59 \\
\hline 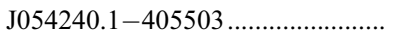 & 914 & 054240.1 & -405503.3 & 51,050 & 3.59 \\
\hline 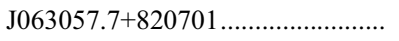 & 1602 & 063057.7 & +820701.2 & 47,933 & 5.27 \\
\hline J090634.4+340055 & 1596 & 090634.4 & +340055.6 & 9907 & 2.28 \\
\hline J091008.4+541852 ....................... & 2227 & 091008.4 & +541852.3 & 107,136 & 1.98 \\
\hline J091126.6+055012 ……................. & 419 & 091126.6 & +055012.5 & 29,165 & 3.70 \\
\hline 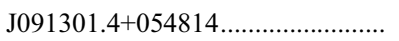 & 419 & 091301.4 & +054814.0 & 29,162 & 3.70 \\
\hline J093102.2+791320 & 839 & 093102.2 & +79 1320.9 & 19,165 & 1.90 \\
\hline J093352.9+552619 & 805 & 093352.9 & +552619.6 & 41,296 & 1.99 \\
\hline 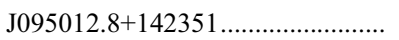 & 2095 & 095012.8 & +142351.7 & 13,962 & 3.13 \\
\hline $\mathrm{J} 101008.7-124013$ & 926 & 101008.7 & -124013.1 & 44,730 & 6.74 \\
\hline J101115.3-124147 ……................... & 926 & 101115.3 & -124147.1 & 44,733 & 6.74 \\
\hline J105624.6-033517 …….................. & 512 & 105624.6 & -033517.4 & 90,211 & 3.67 \\
\hline $\mathrm{J} 111405.8+403157 \ldots \ldots \ldots \ldots \ldots \ldots \ldots$ & 2209 & 111405.8 & +403157.4 & 30,054 & 1.91 \\
\hline J111726.1+074335 …………........ & 363 & 111726.1 & +074335.3 & 26,832 & 4.01 \\
\hline $\mathrm{J} 111730.2+074618 \ldots \ldots \ldots \ldots \ldots \ldots \ldots$ & 363 & 111730.2 & +074618.7 & 26,832 & 4.01 \\
\hline $\mathrm{J} 114008.2-263132 \ldots \ldots \ldots \ldots \ldots \ldots \ldots . .$. & 898 & 114008.2 & -263132.6 & 39,978 & 4.96 \\
\hline 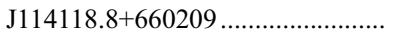 & 536 & 114118.8 & +66 0209.4 & 119,222 & 1.18 \\
\hline $\mathrm{J} 122927.1+752037 \ldots \ldots \ldots \ldots \ldots \ldots \ldots$ & 2253 & 122927.1 & +752037.2 & 48,010 & 2.69 \\
\hline $\mathrm{J} 122940.6+752106 \ldots \ldots \ldots \ldots \ldots \ldots \ldots$ & 2253 & 122940.6 & +752106.6 & 48,010 & 2.73 \\
\hline $\mathrm{J} 131709.9+285513 \ldots \ldots \ldots \ldots \ldots \ldots \ldots$ & 2228 & 131709.9 & +285513.7 & 112,806 & 1.04 \\
\hline $\mathrm{J} 131722.0+285353 \ldots \ldots \ldots \ldots \ldots \ldots$ & 2228 & 131722.0 & +285353.0 & 112,806 & 1.04 \\
\hline $\mathrm{J} 134507.8+000359 \ldots \ldots \ldots \ldots \ldots \ldots \ldots$ & 2251 & 134507.8 & +000359.0 & 9760 & 1.93 \\
\hline 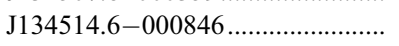 & 2251 & 134514.6 & -000846.5 & 9760 & 1.93 \\
\hline 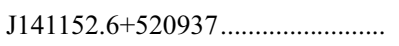 & 2254 & 141152.6 & +520937.2 & 92,102 & 1.34 \\
\hline $\mathrm{J} 141556.8+230727 \ldots \ldots \ldots \ldots \ldots \ldots \ldots . .$. & 2024 & 141556.8 & +230727.1 & 14,755 & 1.91 \\
\hline $\mathrm{J} 141602.1+230647 \ldots \ldots \ldots \ldots \ldots \ldots \ldots$ & 2024 & 141602.1 & +230647.8 & 14,755 & 1.91 \\
\hline $\mathrm{J} 153259.2-004414 \ldots \ldots \ldots \ldots \ldots \ldots \ldots \ldots$ & 2085 & 153259.2 & -004414.7 & 5152 & 6.25 \\
\hline $\mathrm{J} 153415.0+232459 \ldots \ldots \ldots \ldots \ldots \ldots$ & 869 & 153415.0 & +232459.7 & 57,181 & 4.28 \\
\hline $\mathrm{J} 154932.0+213300 \ldots \ldots \ldots \ldots \ldots \ldots$ & 326 & 154932.0 & +213300.7 & 42,688 & 4.30 \\
\hline $\mathrm{J} 160847.1+654139 \ldots \ldots \ldots \ldots \ldots \ldots \ldots$ & 2127 & 160847.1 & +654139.2 & 44,648 & 2.83 \\
\hline 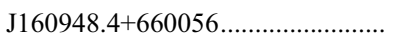 & 2127 & 160948.4 & +660056.9 & 44,648 & 2.83 \\
\hline J165514.4-082944 ………........... & 615 & 165514.4 & -082944.0 & 9152 & 13.40 \\
\hline J205537.4-043334 ……................ & 551 & 205537.4 & -043334.8 & 44,880 & 4.96 \\
\hline J205617.2-044154 _....................... & 551 & 205617.2 & -044154.8 & 44,880 & 4.96 \\
\hline 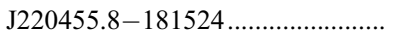 & 2114 & 220455.8 & -181524.3 & 5146 & 2.79 \\
\hline $\mathrm{J} 221326.2-220532 \ldots \ldots \ldots \ldots \ldots \ldots \ldots$ & 1479 & 221326.2 & -220532.4 & 20,774 & 2.49 \\
\hline $\mathrm{J} 223538.4+340609 \ldots \ldots \ldots \ldots \ldots \ldots \ldots \ldots$ & 789 & 223538.4 & +340609.3 & 19,955 & 7.74 \\
\hline $\mathrm{J} 223614.5+335648 \ldots \ldots \ldots \ldots \ldots \ldots \ldots . .$. & 789 & 223614.5 & +335648.4 & 19,955 & 7.74 \\
\hline $\mathrm{J} 230150.7+084352 \ldots \ldots \ldots \ldots \ldots \ldots \ldots . .$. & 918 & 230150.7 & +084352.5 & 109,955 & 5.05 \\
\hline $\mathrm{J} 230227.7+083901 \ldots \ldots \ldots \ldots \ldots \ldots \ldots$ & 918 & 230227.7 & +083901.4 & 109,955 & 5.05 \\
\hline $\mathrm{J} 230252.0+084137 \ldots \ldots \ldots \ldots \ldots \ldots \ldots . .$. & 918 & 230252.0 & +084137.0 & 109,955 & 5.05 \\
\hline $\mathrm{J} 230311.1+085131 \ldots \ldots \ldots \ldots \ldots \ldots \ldots$ & 918 & 230311.1 & +085131.2 & 109,955 & 5.05 \\
\hline $\mathrm{J} 234817.8+010617 \ldots \ldots \ldots \ldots \ldots \ldots \ldots$ & 861 & 234817.8 & +010617.2 & 37,322 & 3.81 \\
\hline
\end{tabular}

Note.-Units of right ascension are hours, minutes, and seconds, and units of declination are degrees, arcminutes, and arcseconds.

${ }^{a}$ Vignetting-corrected exposure time.

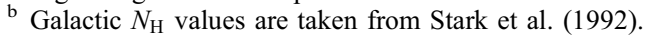


TABLE 2

Optical Mosaic Fields

\begin{tabular}{|c|c|c|c|c|c|c|c|c|c|}
\hline ObsID & $E(B-V)^{\mathrm{a}}$ & Telescope & UT Date & Filter & Dithers & $\begin{array}{c}\text { Total Exposure } \\
\text { (s) }\end{array}$ & $\begin{array}{c}\text { Air Mass } \\
\text { (mean) }\end{array}$ & $\begin{array}{l}\text { FWHM }^{\mathrm{b}} \\
(\operatorname{arcsec})\end{array}$ & $\begin{array}{l}M_{\mathrm{TO}}{ }^{\mathrm{c}} \\
(\mathrm{mag})\end{array}$ \\
\hline \multirow[t]{3}{*}{$326 \ldots$} & 0.046 & KPNO $4 \mathrm{~m}$ & 2001 Jun 13 & $g^{\prime}$ & 3 & 2100 & 1.05 & 1.1 & 24.88 \\
\hline & & & & $r^{\prime}$ & 5 & 2000 & 1.15 & 1.3 & 24.38 \\
\hline & & & 2001 Jun 12 & $i^{\prime}$ & 15 & 4500 & 1.18 & 1.1 & 24.62 \\
\hline \multirow[t]{3}{*}{ 363 } & 0.041 & $\mathrm{KPNO} 4 \mathrm{~m}$ & 2001 Jun 13 & $g^{\prime}$ & 2 & 1200 & 2.26 & 1.8 & 23.62 \\
\hline & & & & $r^{\prime}$ & 5 & 1500 & 1.94 & 1.6 & 23.88 \\
\hline & & & & $i^{\prime}$ & 5 & 1500 & 1.46 & 1.3 & 23.12 \\
\hline \multirow[t]{3}{*}{ 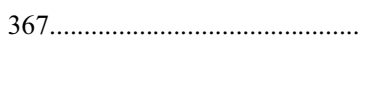 } & 0.047 & KPNO $4 \mathrm{~m}$ & 2004 Jun 19 & $g^{\prime}$ & 3 & 900 & 1.50 & 1.3 & 24.38 \\
\hline & & & & $r^{\prime}$ & 3 & 600 & 1.40 & 1.7 & 23.62 \\
\hline & & & & $i^{\prime}$ & 3 & 600 & 1.32 & 1.1 & 23.88 \\
\hline \multirow[t]{3}{*}{ 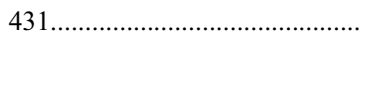 } & 0.071 & KPNO $4 \mathrm{~m}$ & 2000 Jun 11 & $g^{\prime}$ & 2 & 1000 & 1.36 & 1.6 & 24.12 \\
\hline & & & & $r^{\prime}$ & 1 & 500 & 1.28 & 1.6 & 23.38 \\
\hline & & & & $i^{\prime}$ & 1 & 360 & 1.25 & 1.2 & 22.88 \\
\hline \multirow[t]{3}{*}{ 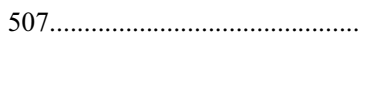 } & 0.061 & CTIO $4 \mathrm{~m}$ & 2003 Apr 7 & $g^{\prime}$ & 3 & 900 & 1.06 & 1.0 & 24.88 \\
\hline & & & & $r^{\prime}$ & 3 & 600 & 1.08 & 1.0 & 24.38 \\
\hline & & & & $i^{\prime}$ & 3 & 600 & 1.09 & 1.1 & 23.62 \\
\hline \multirow[t]{3}{*}{ 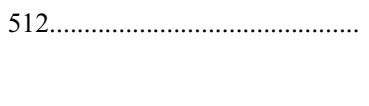 } & 0.034 & KPNO $4 \mathrm{~m}$ & 2001 Feb 22 & $g^{\prime}$ & 5 & 4500 & 1.24 & 1.3 & 24.88 \\
\hline & & & & $r^{\prime}$ & 3 & 2400 & 1.24 & 1.1 & 24.38 \\
\hline & & & & $i^{\prime}$ & 5 & 2000 & 1.32 & 1.3 & 23.62 \\
\hline \multirow{3}{*}{ 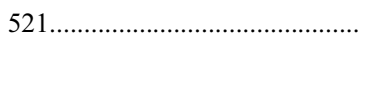 } & 0.061 & $\mathrm{KPNO} 4 \mathrm{~m}$ & 2001 Oct 24 & $g^{\prime}$ & 5 & 3000 & 1.15 & 1.5 & 24.62 \\
\hline & & & & $r^{\prime}$ & 5 & 3000 & 1.04 & 1.2 & 24.38 \\
\hline & & & & $i^{\prime}$ & 5 & 3000 & 1.00 & 1.1 & 23.88 \\
\hline \multirow[t]{3}{*}{ 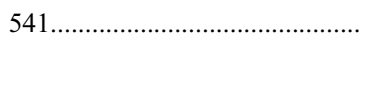 } & 0.007 & KPNO $4 \mathrm{~m}$ & 2000 Jun 12 & $g^{\prime}$ & 2 & 1000 & 1.33 & 1.9 & 22.88 \\
\hline & & & & $r^{\prime}$ & 1 & 500 & 1.41 & 2.0 & 22.12 \\
\hline & & & & $i^{\prime}$ & 1 & 500 & 1.50 & 1.7 & 22.12 \\
\hline \multirow[t]{3}{*}{ 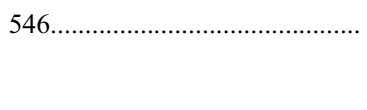 } & 0.035 & KPNO $4 \mathrm{~m}$ & 2000 Jun 11 & $g^{\prime}$ & 2 & 1400 & 1.04 & 1.5 & 23.62 \\
\hline & & & & $r^{\prime}$ & 1 & 500 & 1.02 & 1.5 & 22.88 \\
\hline & & & & $i^{\prime}$ & 1 & 500 & 1.02 & 1.2 & 23.12 \\
\hline \multirow[t]{3}{*}{ 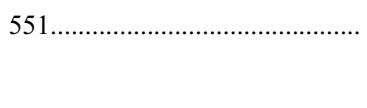 } & 0.079 & $\mathrm{KPNO} 4 \mathrm{~m}$ & 2000 Oct 17 & $g^{\prime}$ & 2 & 1800 & 1.27 & 1.4 & 24.88 \\
\hline & & & & $r^{\prime}$ & 3 & 1440 & 1.40 & 1.1 & 24.62 \\
\hline & & & & $i^{\prime}$ & 3 & 1260 & 1.52 & 1.4 & 23.62 \\
\hline \multirow[t]{3}{*}{ 796 } & 0.046 & KPNO $4 \mathrm{~m}$ & 2001 Oct 24 & $g^{\prime}$ & 3 & 2700 & 1.38 & 1.1 & 25.12 \\
\hline & & & & $r^{\prime}$ & 3 & 2400 & 1.61 & 1.2 & 24.62 \\
\hline & & & & $i^{\prime}$ & 3 & 1200 & 1.30 & 1.1 & 23.62 \\
\hline 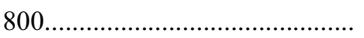 & 0.019 & KPNO $4 \mathrm{~m}$ & 2001 Jun 14 & $g^{\prime}$ & 3 & 2400 & 1.11 & 1.8 & 24.62 \\
\hline & & & & $r^{\prime}$ & 3 & 2100 & 1.28 & 1.6 & 23.88 \\
\hline & & & & $i^{\prime}$ & 7 & 2520 & 1.48 & 1.3 & 23.62 \\
\hline 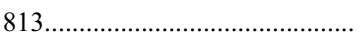 & 0.015 & CTIO $4 \mathrm{~m}$ & 2000 Sep 30 & $g^{\prime}$ & 3 & 180 & 1.07 & 1.4 & 23.88 \\
\hline & & & & $r^{\prime}$ & 2 & 120 & 1.09 & 1.3 & 23.62 \\
\hline & & & & $i^{\prime}$ & 3 & 180 & 1.11 & 1.2 & 22.88 \\
\hline 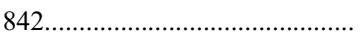 & 0.058 & CTIO $4 \mathrm{~m}$ & 2000 Sep 30 & $g^{\prime}$ & 3 & 180 & 1.06 & 1.1 & 23.62 \\
\hline & & & & $r^{\prime}$ & 3 & 180 & 1.06 & 1.0 & 23.62 \\
\hline & & & & $i^{\prime}$ & 3 & 180 & 1.06 & 1.0 & 22.62 \\
\hline 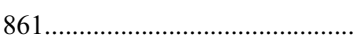 & 0.025 & CTIO $4 \mathrm{~m}$ & 2000 Sep 29 & $g^{\prime}$ & 3 & 1260 & 1.24 & 1.4 & 24.62 \\
\hline & & & & $r^{\prime}$ & 3 & 1080 & 1.17 & 1.4 & 24.38 \\
\hline & & & & $i^{\prime}$ & 3 & 1170 & 1.17 & 1.1 & 23.62 \\
\hline 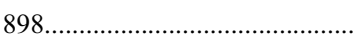 & 0.038 & CTIO $4 \mathrm{~m}$ & 2003 Apr 7 & $g^{\prime}$ & 3 & 1800 & 1.02 & 1.0 & 25.38 \\
\hline & & & & $r^{\prime}$ & 3 & 1200 & 1.06 & 1.0 & 24.62 \\
\hline & & & & $i^{\prime}$ & 3 & 900 & 1.10 & 0.8 & 23.88 \\
\hline 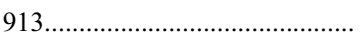 & 0.014 & KPNO $4 \mathrm{~m}$ & 2001 Oct 23 & $g^{\prime}$ & 5 & 3500 & 1.46 & 1.1 & 25.12 \\
\hline & & & & $r^{\prime}$ & 5 & 3000 & 1.44 & 1.0 & 24.62 \\
\hline & & & & $i^{\prime}$ & 5 & 2000 & 1.52 & 1.1 & 23.62 \\
\hline 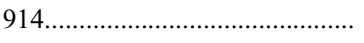 & 0.036 & CTIO $4 \mathrm{~m}$ & 2000 Sep 29 & $g^{\prime}$ & 3 & 990 & 1.04 & 1.2 & 25.12 \\
\hline & & & & $r^{\prime}$ & 3 & 810 & 1.06 & 1.2 & 24.38 \\
\hline & & & & $i^{\prime}$ & 3 & 900 & 1.08 & 1.2 & 23.62 \\
\hline 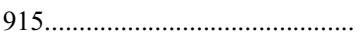 & 0.051 & CTIO $4 \mathrm{~m}$ & 2003 Apr 7 & $g^{\prime}$ & 3 & 1800 & 1.02 & 1.0 & 25.38 \\
\hline & & & & $r^{\prime}$ & 3 & 1200 & 1.00 & 1.0 & 24.62 \\
\hline & & & & $i^{\prime}$ & 3 & 900 & 1.00 & 0.8 & 23.88 \\
\hline 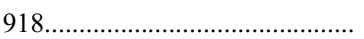 & 0.081 & KPNO $4 \mathrm{~m}$ & 2001 Oct 23 & $g^{\prime}$ & 5 & 4500 & 1.13 & 1.2 & 24.88 \\
\hline & & & & $r^{\prime}$ & 5 & 3000 & 1.09 & 1.3 & 24.38 \\
\hline & & & & $i^{\prime}$ & 5 & 1500 & 1.14 & 1.2 & 23.38 \\
\hline 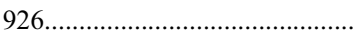 & 0.071 & CTIO $4 \mathrm{~m}$ & 2003 Apr 6 & $g^{\prime}$ & 3 & 1800 & 1.15 & 1.1 & 24.88 \\
\hline & & & 2003 Apr 7 & $r^{\prime}$ & 3 & 1200 & 1.10 & 1.2 & 24.38 \\
\hline & & & 2003 Apr 7 & $i^{\prime}$ & 3 & 900 & 1.07 & 0.9 & 23.88 \\
\hline 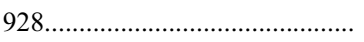 & 0.052 & CTIO $4 \mathrm{~m}$ & 2000 Sep 29 & $g^{\prime}$ & 3 & 900 & 1.01 & 1.6 & 24.38 \\
\hline & & & & $r^{\prime}$ & 3 & 720 & 1.02 & 1.3 & 24.12 \\
\hline
\end{tabular}


TABLE 2-Continued

\begin{tabular}{|c|c|c|c|c|c|c|c|c|c|}
\hline ObsID & $E(B-V)^{\mathrm{a}}$ & Telescope & UT Date & Filter & Dithers & $\begin{array}{c}\text { Total Exposure } \\
\text { (s) }\end{array}$ & $\begin{array}{l}\text { Air Mass } \\
\text { (mean) }\end{array}$ & $\begin{array}{l}\text { FWHM }^{\mathrm{b}} \\
(\operatorname{arcsec})\end{array}$ & $\begin{array}{l}M_{\mathrm{TO}}{ }^{\mathrm{c}} \\
(\mathrm{mag})\end{array}$ \\
\hline & & & & $i^{\prime}$ & 3 & 810 & 1.04 & 1.0 & 23.62 \\
\hline \multirow[t]{3}{*}{ 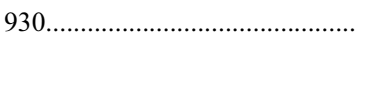 } & 0.021 & KPNO $4 \mathrm{~m}$ & 2001 Feb 22 & $g^{\prime}$ & 3 & 2700 & 1.11 & 1.2 & 25.62 \\
\hline & & & & $r^{\prime}$ & 3 & 2550 & 1.07 & 1.3 & 24.62 \\
\hline & & & & $i^{\prime}$ & 4 & 2080 & 1.08 & 1.2 & 24.38 \\
\hline \multirow[t]{3}{*}{1479} & 0.033 & CTIO $4 \mathrm{~m}$ & 2001 Aug 22 & $g^{\prime}$ & 2 & 1200 & 1.21 & 1.0 & 24.88 \\
\hline & & & & $r^{\prime}$ & 2 & 900 & 1.39 & 1.0 & 24.38 \\
\hline & & & & $i^{\prime}$ & 2 & 720 & 1.44 & 1.1 & 23.62 \\
\hline \multirow[t]{3}{*}{$1602 .}$. & 0.080 & KPNO $4 \mathrm{~m}$ & 2001 Oct 23 & $g^{\prime}$ & 1 & 800 & 1.56 & 1.4 & 24.38 \\
\hline & & & & $r^{\prime}$ & 1 & 750 & 1.56 & 1.1 & 23.88 \\
\hline & & & & $i^{\prime}$ & 1 & 400 & 1.56 & 1.0 & 23.12 \\
\hline \multirow[t]{3}{*}{ 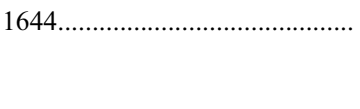 } & 0.030 & CTIO $4 \mathrm{~m}$ & 2001 Aug 9 & $g^{\prime}$ & 5 & 1805 & 1.08 & 1.0 & 24.38 \\
\hline & & & & $r^{\prime}$ & 3 & 810 & 1.09 & 1.0 & 23.88 \\
\hline & & & & $i^{\prime}$ & 3 & 570 & 1.10 & 0.9 & 23.62 \\
\hline \multirow[t]{3}{*}{ 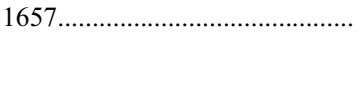 } & 0.027 & $\mathrm{KPNO} 4 \mathrm{~m}$ & 2004 Jun 17 & $g^{\prime}$ & 3 & 1800 & 1.08 & 1.1 & 24.88 \\
\hline & & & & $r^{\prime}$ & 3 & 1200 & 1.16 & 1.0 & 24.38 \\
\hline & & & & $i^{\prime}$ & 3 & 900 & 1.24 & 1.2 & 23.62 \\
\hline \multirow[t]{3}{*}{ 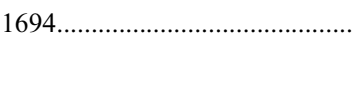 } & 0.065 & KPNO $4 \mathrm{~m}$ & 2004 Jun 17 & $g^{\prime}$ & 3 & 1800 & 1.54 & 1.1 & 24.62 \\
\hline & & & & $r^{\prime}$ & 3 & 1200 & 1.36 & 1.1 & 24.38 \\
\hline & & & & $i^{\prime}$ & 3 & 900 & 1.28 & 0.9 & 23.62 \\
\hline \multirow[t]{3}{*}{ 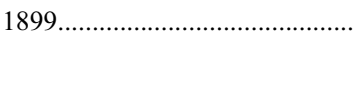 } & 0.041 & KPNO $4 \mathrm{~m}$ & 2004 Jun 17 & $g^{\prime}$ & 3 & 1800 & 1.03 & 1.0 & 25.12 \\
\hline & & & & $r^{\prime}$ & 3 & 1200 & 1.03 & 1.0 & 24.62 \\
\hline & & & & $i^{\prime}$ & 3 & 900 & 1.04 & 0.8 & 24.12 \\
\hline \multirow[t]{3}{*}{ 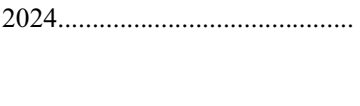 } & 0.024 & KPNO $4 \mathrm{~m}$ & 2004 Jun 19 & $g^{\prime}$ & 3 & 900 & 1.17 & 0.9 & 24.88 \\
\hline & & & & $r^{\prime}$ & 3 & 600 & 1.24 & 1.0 & 24.38 \\
\hline & & & & $i^{\prime}$ & 3 & 600 & 1.30 & 0.9 & 23.88 \\
\hline \multirow[t]{3}{*}{ 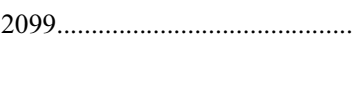 } & 0.044 & KPNO $4 \mathrm{~m}$ & 2001 Dec 14 & $g^{\prime}$ & 1 & 100 & 1.21 & 2.1 & 22.88 \\
\hline & & & & $r^{\prime}$ & 1 & 90 & 1.21 & 1.6 & 22.38 \\
\hline & & & & $i^{\prime}$ & 1 & 85 & 1.21 & 1.3 & 21.38 \\
\hline \multirow[t]{3}{*}{ 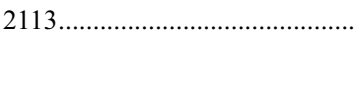 } & 0.026 & CTIO $4 \mathrm{~m}$ & 2001 Aug 9 & $g^{\prime}$ & 1 & 400 & 1.06 & 1.0 & 23.62 \\
\hline & & & & $r^{\prime}$ & 1 & 150 & 1.07 & 0.9 & 23.12 \\
\hline & & & & $i^{\prime}$ & 1 & 120 & 1.08 & 0.9 & 22.12 \\
\hline \multirow[t]{3}{*}{ 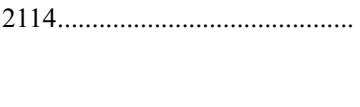 } & 0.030 & CTIO $4 \mathrm{~m}$ & 2001 Aug 9 & $g^{\prime}$ & 1 & 400 & 1.30 & 1.1 & 23.12 \\
\hline & & & & $r^{\prime}$ & 1 & 150 & 1.34 & 1.1 & 22.88 \\
\hline & & & & $i^{\prime}$ & 1 & 120 & 1.28 & 0.9 & 22.12 \\
\hline \multirow[t]{3}{*}{ 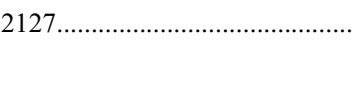 } & 0.034 & KPNO $4 \mathrm{~m}$ & 2004 Jun 19 & $g^{\prime}$ & 3 & 1800 & 1.36 & 1.4 & 24.88 \\
\hline & & & & $r^{\prime}$ & 3 & 1200 & 1.43 & 1.1 & 24.62 \\
\hline & & & & $i^{\prime}$ & 3 & 900 & 1.54 & 1.4 & 23.62 \\
\hline \multirow[t]{3}{*}{ 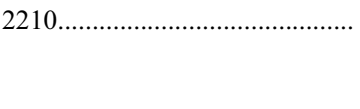 } & 0.014 & KPNO $4 \mathrm{~m}$ & 2004 Jun 18 & $g^{\prime}$ & 3 & 1800 & 1.15 & 1.7 & 24.38 \\
\hline & & & & $r^{\prime}$ & 3 & 1200 & 1.10 & 1.4 & 24.12 \\
\hline & & & & $i^{\prime}$ & 3 & 900 & 1.07 & 1.2 & 23.62 \\
\hline \multirow[t]{3}{*}{ 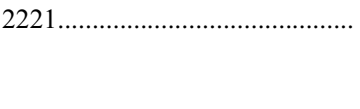 } & 0.020 & KPNO $4 \mathrm{~m}$ & 2004 Jun 18 & $g^{\prime}$ & 3 & 1800 & 1.20 & 1.4 & 25.12 \\
\hline & & & & $r^{\prime}$ & 3 & 1200 & 1.15 & 1.4 & 24.38 \\
\hline & & & & $i^{\prime}$ & 3 & 900 & 1.12 & 1.2 & 23.88 \\
\hline \multirow[t]{3}{*}{ 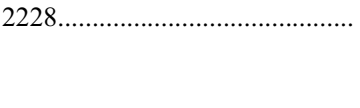 } & 0.009 & $\mathrm{KPNO} 4 \mathrm{~m}$ & 2004 Jun 19 & $g^{\prime}$ & 3 & 1800 & 1.07 & 0.9 & 25.12 \\
\hline & & & & $r^{\prime}$ & 3 & 1200 & 1.13 & 0.8 & 24.88 \\
\hline & & & & $i^{\prime}$ & 3 & 900 & 1.20 & 0.9 & 24.12 \\
\hline
\end{tabular}

${ }^{\text {a }}$ Galactic extinction values are calculated from the maps of Schlegel et al. (1998).

${ }^{\mathrm{b}}$ FWHM of point sources in final stacked image.

c Turnover magnitude of galaxy counts using 0.25 mag bins prior to extinction correction.

was used to assemble a final list consisting of 55 high-confidence serendipitously detected extended sources (see Table 1 and Fig. 1). From the sample of 55 extended X-ray sources, 6 were found to be associated with low-redshift galaxies (3 ellipticals, 2 spirals, and $1 \mathrm{~S} 0 / \mathrm{Sa}$ galaxy).

The X-ray flux for each source was computed from the total number of counts by extrapolating the $\beta$ model fit to infinity. Also assumed was a Raymond-Smith thermal spectrum with a temperature of $T_{\mathrm{X}}=2 \mathrm{keV}$, a solar abundance of $Z_{\odot}=0.3$, and Galactic extinction appropriate for each field. We use $T_{\mathrm{X}}=$ $2 \mathrm{keV}$ since it is appropriate based on the median $L_{\mathrm{X}}$ of our cluster sample $\left(L_{\mathrm{X}} \sim 10^{43} \mathrm{ergs} \mathrm{s}^{-1}\right)$ and the $T_{\mathrm{X}}-L_{\mathrm{X}}$ relation (e.g.,
White et al. 1997). Using $T_{\mathrm{X}}=5 \mathrm{keV}$, for example, will change $f_{\mathrm{X}}$ by $\sim 8 \%$.

X-ray flux values were converted to the $0.5-2 \mathrm{keV}$ energy band and uncertainties derived from Poisson statistics. X-ray luminosities were calculated from measured fluxes using redshift estimates derived from (in order of preference): (1) the ChaMP spectroscopic program (Green et al. 2004) or (2) published spectroscopic redshifts or (3) were estimated from our red-sequencefiltered VTP optical cluster detection method (see $\S 3.2)$. In Table 3 the X-ray properties of our extended source catalog are tabulated. Figure 2 shows the all-sky distribution of our final sample of 55 extended X-ray sources. 


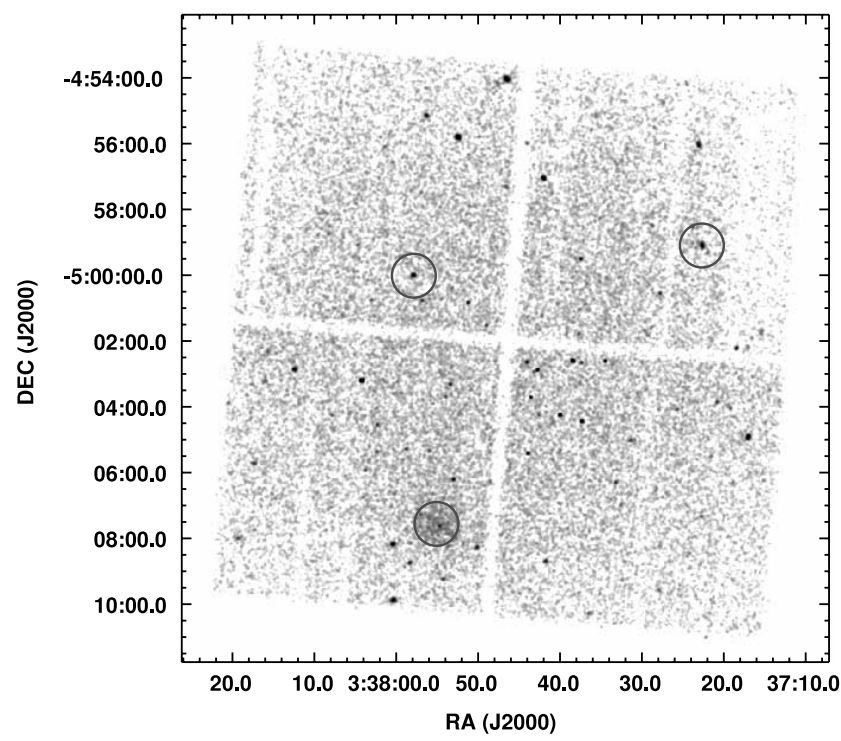

FIG. 1.-Part of a smoothed Chandra image, ObsID 796, showing the location of three serendipitously detected extended X-ray sources on three ACIS-I chips (circles; $40^{\prime \prime}$ in radius). The PI target, the blue compact dwarf galaxy SBS $0335-052$, is located near the center of the image.

\subsection{Optical Cluster Detection}

The detection of galaxy clusters from optical data has had a long history dating back to the pioneering work of Abell (1958) and Zwicky et al. (1961). Various techniques have been used to take advantage of the expected shape, luminosity function, density enhancement, and color distribution of the cluster galaxy population. Some of the automated methods include the matched-filter algorithm (Postman et al. 1996), the VTP method (Ebeling \& Wiedenmann 1993; Ramella et al. 2001), the cluster red-sequence technique (Gladders \& Yee 2000), the detection of surface brightness fluctuations (Gonzalez et al. 2001), and the maxBCG procedure (Bahcall et al. 2003). The properties of the resulting cluster samples naturally differ, depending on the data quality, the detection technique, and selection criteria (e.g., Donahue et al. 2002; Kim et al. 2002).

\subsubsection{Red-Sequence Voronoi Tessellation and Percolation Algorithm}

The construction of a cluster catalog from ChaMP optical images is based on a modified version of the VTP technique described in Ebeling \& Wiedenmann (1993) and Ramella et al. (2001). ${ }^{11}$ The advantage of implementing this type of nonparametric algorithm is that no assumption is made regarding cluster shapes - as is the case for the matched-filter code - and thus VTP is sensitive to irregular clusters as well as symmetric ones.

The VTP algorithm partitions the galaxy spatial plane into polyhedral cells, each containing a single unique galaxy (Voronoi cell). The cell size is determined by the distance between nearest neighbors and encloses the maximum area nearest to a given galaxy (see Fig. 3). Galaxy clusters are detected as overdensities in the number of Voronoi cells (grouped together using a percolation technique) per unit area (Ramella et al. 2001). A slightly modified approach is to use the inverse of the area contained within each Voronoi cell (Ebeling \& Wiedenmann 1993; Kim et al. 2002). The significance of each galaxy overdensity is computed by comparing the density distribution of the galaxy catalog with that expected for a random distribution of Voronoi

11 The VTP code was downloaded from http://www.ts.astro.it/astro/VoroHome. cells - the so-called Kiang distribution (Kiang 1966). Overdense regions composed of adjacent Voronoi cells are flagged as potential clusters if their density is greater than a specified threshold. A random fluctuation in the background can potentially exceed the imposed threshold constraint and thus be counted as a real cluster. This contamination is minimized by computing the probability (based on simulations) that a given detection is a random background fluctuation and then only including regions above an acceptable level (see $\S$ 3.2.2).

To improve the contrast of cluster galaxies with respect to the background field population, we have implemented a refined version of the VTP method that takes advantage of the existence of the red sequence in the color-magnitude relation of early-type cluster galaxies (e.g., Baum 1959; Sandage \& Visvanathan 1978; López-Cruz et al. 2004). The red sequence for early-type cluster galaxies shifts to progressively redder observed colors as the $4000 \AA$ break moves through the filter bandpasses with increasing redshift (i.e., the K-correction; Humason et al. 1956; Oke \& Sandage 1968). The position of the cluster red sequence in the colormagnitude plane can be used as an estimator of redshift (Gladders \& Yee 2000; López-Cruz et al. 2004). Thus, choosing appropriate filters enables foreground and background galaxies to be culled to minimize the contamination from the field galaxy population. As an example, Kim et al. (2002) applied the VTP algorithm to SDSS galaxy catalogs constructed by selecting galaxies relative to the expected red sequence in the $g^{*}-r^{*}$ versus $r^{*}$ color-magnitude plane for clusters at various redshifts. The color width blueward of the red sequence for each redshift slice was chosen to be relatively broad $\left[\Delta\left(g^{*}-r^{*}\right) \sim 0.6\right.$ mag; see their Fig. 2].

The ChaMP optical data consist of magnitudes measured in the $g^{\prime}, r^{\prime}$, and $i^{\prime}$ bands. Since we are interested in assembling a cluster sample that extends to high redshift $(z>0.5)$, we have elected to use the $r^{\prime}-i^{\prime}$ color to select galaxies, since $g^{\prime}-r^{\prime}$ becomes degenerate at $z \lesssim 0.4$ (T. Kodama 2004, private communication). The $r^{\prime}-i^{\prime}$ color allows us to sample cluster red sequences out to $z \sim 0.7$ (see Fig. 4). The basic procedure is to construct catalogs containing galaxies with a $r^{\prime}-i^{\prime}$ color distribution that matches a particular red sequence for a given redshift. Catalogs are produced for red sequences that sample the redshift range from $z=0.05-0.70$. VTP is then applied to each galaxy catalog, and the most significant detections (as flagged by VTP) are included in the final cluster compilation for a given field. The advantage of this technique over the standard VTP method (e.g., Ramella et al. 2001) is that the "noise" from field galaxies is reduced and also that the redshift of the detected cluster can be estimated from the catalog yielding the greatest detection significance. Several recent studies have been successful in using color cuts relative to the red sequence to search for clusters using optical data (e.g., Gladders \& Yee 2000; Goto et al. 2002; Nichol 2004; Hsieh et al. 2005).

For the location of the red sequence in the color-magnitude plane, we adopt the models of Kodama \& Arimoto (1997) transformed to the SDSS filters ( T. Kodama 2004, private communication). Each galaxy catalog is generated for a specific red sequence by selecting galaxies with a $r^{\prime}-i^{\prime}$ color within \pm 0.1 mag of the red-sequence line (all galaxies are corrected for galactic extinction prior to the selection process; see Table 2). We choose a color width of 0.1 mag either side of the red sequence, since the measured dispersion of cluster galaxies along the red sequence is $\sim 0.07$ mag (e.g., Bower et al. 1992; Ellis et al. 1997; LópezCruz et al. 2004). To ensure that our galaxy catalogs sample the complete range in color for our expected cluster redshift distribution, we construct galaxy samples for 27 red sequences from $z=$ $0.05-0.70$ (Fig. 4). The density of these model red sequences in 
TABLE 3

X-Ray Properties of Extended Sources

\begin{tabular}{|c|c|c|c|c|c|c|c|c|c|c|}
\hline $\begin{array}{c}\text { Source Name } \\
\text { CXOMP }\end{array}$ & Counts & $\begin{array}{c}f_{\mathrm{X}} \\
\left(10^{-14} \mathrm{cgs}\right)\end{array}$ & $\begin{array}{c}\Delta f_{\mathrm{X}}{ }^{\mathrm{a}} \\
\left(10^{-14} \text { cgs }\right)\end{array}$ & $\begin{array}{c}L_{\mathrm{X}}^{\mathrm{b}} \\
\left(10^{42} \mathrm{cgs}\right)\end{array}$ & $\begin{array}{c}\Delta L_{\mathrm{X}} \\
\left(10^{42} \mathrm{cgs}\right)\end{array}$ & $\begin{array}{c}r_{c}^{\mathrm{c}} \\
(\operatorname{arcsec})\end{array}$ & $\begin{array}{c}\operatorname{PSF}^{\mathrm{d}} \\
(\operatorname{arcsec})\end{array}$ & $\begin{array}{c}\mathrm{OAA}^{\mathrm{e}} \\
(\operatorname{arcmin})\end{array}$ & $\begin{array}{c}z^{\mathrm{f}} \\
\text { (spec) }\end{array}$ & $\begin{array}{c}z^{\mathrm{g}} \\
(\mathrm{VTP})\end{array}$ \\
\hline J002650.2+171935 „................ & 125.38 & 2.523 & 0.225 & 23.161 & 2.068 & 15.98 & 5.41 & 9.68 & 0.4907 & $\ldots$ \\
\hline J005848.1-280035 ................ & 178.32 & 15.272 & 1.144 & 17.967 & 1.346 & 24.90 & 7.65 & 11.52 & 0.2021 & $\ldots$ \\
\hline J010214.1+314915 „................. & 124.91 & 1.593 & 0.142 & 2.429 & 0.217 & 14.03 & 0.63 & 2.57 & $\ldots$ & 0.227 \\
\hline J010607.0+004943 .................. & 186.98 & 23.209 & 1.697 & 55.593 & 4.066 & 40.34 & 0.76 & 3.14 & 0.2767 & $\ldots$ \\
\hline J010610.3+005126 _................. & 120.07 & 22.936 & 2.093 & 48.930 & 4.465 & 41.15 & 0.81 & 3.32 & 0.2630 & $\ldots$ \\
\hline J013642.6+204843 ..................... & 229.06 & 3.632 & 0.240 & $\ldots$ & $\ldots$ & 27.45 & 4.84 & 9.16 & $\ldots$ & $\ldots$ \\
\hline J033639.4-045515 ................. & 600.58 & 13.281 & 0.542 & $\ldots$ & $\ldots$ & 39.28 & 17.96 & 17.67 & $\ldots$ & $\ldots$ \\
\hline J033722.6-045906 ................. & 166.37 & 2.822 & 0.219 & 0.022 & 0.002 & 3.07 & 2.33 & 6.29 & 0.0185 & $\ldots$ \\
\hline J033755.1-050733 „................ & 2770.02 & 39.048 & 0.742 & 12.687 & 0.241 & 55.46 & 2.05 & 5.88 & 0.1123 & 0.102 \\
\hline J033757.8-050001 …................ & 64.83 & 0.889 & 0.110 & 0.026 & 0.003 & 2.32 & 1.07 & 4.06 & $0.0357^{\mathrm{h}}$ & $\ldots$ \\
\hline J040351.2-170823 „................. & 407.08 & 55.282 & 2.740 & $\ldots$ & $\ldots$ & 81.79 & 1.93 & 5.69 & $\ldots$ & $\ldots$ \\
\hline J054152.7-410702 „................. & 245.74 & 3.877 & 0.247 & $\ldots$ & $\ldots$ & 9.96 & 10.39 & 13.44 & $\ldots$ & $\ldots$ \\
\hline J054240.1-405503 ................. & 273.41 & 3.582 & 0.217 & 61.071 & 3.693 & 21.37 & 1.09 & 4.10 & 0.6340 & 0.627 \\
\hline J063057.7+820701 .................... & 201.10 & 4.166 & 0.294 & 9.458 & 0.667 & 35.50 & 6.93 & 10.96 & $0.2703^{\mathrm{h}}$ & 0.302 \\
\hline J090634.4+340055 ................... & 74.72 & 7.081 & 0.819 & 25.292 & 2.926 & 19.64 & 13.28 & 15.19 & 0.3290 & $\ldots$ \\
\hline J091008.4+541852 „................ & 2427.33 & 15.172 & 0.308 & $\ldots$ & $\ldots$ & 1.24 & 1.35 & 4.68 & $\ldots$ & $\ldots$ \\
\hline J091126.6+055012 _................. & 175.64 & 2.902 & 0.219 & 79.135 & 5.971 & 13.34 & 0.50 & 1.01 & 0.7682 & $\ldots$ \\
\hline J091301.4+054814 „.................. & 254.94 & 8.056 & 0.504 & $\ldots$ & $\ldots$ & 39.71 & 31.32 & 23.34 & $\ldots$ & $\ldots$ \\
\hline J093102.2+791320 .................. & 1384.00 & 43.783 & 1.177 & 384.842 & 10.345 & 30.19 & 3.32 & 7.56 & $0.4819^{\mathrm{h}}$ & $\ldots$ \\
\hline J093352.9+552619 „.................. & 132.45 & 2.415 & 0.210 & $\ldots$ & $\ldots$ & 14.62 & 7.87 & 11.69 & $\ldots$ & $\ldots$ \\
\hline J095012.8+142351 „.................. & 164.93 & 5.466 & 0.426 & $\ldots$ & $\ldots$ & 17.30 & 1.26 & 4.48 & $\ldots$ & $\ldots$ \\
\hline $\mathrm{J} 101008.7-124013 \ldots \ldots \ldots \ldots \ldots \ldots$ & 71.75 & 1.100 & 0.130 & $\ldots$ & $\ldots$ & 15.47 & 0.52 & 1.68 & $\ldots$ & $\ldots$ \\
\hline J101115.3-124147 _................. & 373.72 & 6.929 & 0.358 & $\ldots$ & $\ldots$ & 16.35 & 12.62 & 14.81 & $\ldots$ & $\ldots$ \\
\hline J105624.6-033517 ................ & 705.80 & 6.058 & 0.228 & 100.132 & 3.769 & 36.23 & 4.44 & 8.76 & 0.6260 & 0.602 \\
\hline $\mathrm{J} 111405.8+403157 \ldots \ldots \ldots \ldots \ldots \ldots \ldots$ & 61.29 & 1.559 & 0.199 & $\ldots$ & $\ldots$ & 23.12 & 2.88 & 7.03 & $\ldots$ & $\ldots$ \\
\hline $\mathrm{J} 111726.1+074335 \ldots \ldots \ldots \ldots \ldots \ldots$ & 286.03 & 9.866 & 0.583 & 84.623 & 5.004 & 32.88 & 8.70 & 12.29 & 0.4770 & 0.552 \\
\hline $\mathrm{J} 111730.2+074618 \ldots \ldots \ldots \ldots \ldots \ldots$ & 165.91 & 5.397 & 0.419 & 3.783 & 0.294 & 34.48 & 7.23 & 11.20 & 0.1600 & 0.177 \\
\hline $\mathrm{J} 114008.2-263132 \ldots \ldots \ldots \ldots \ldots \ldots$ & 233.82 & 5.119 & 0.335 & 111.844 & 7.314 & 33.85 & 4.23 & 8.55 & $\ldots$ & 0.702 \\
\hline $\mathrm{J} 114118.8+660209 \ldots \ldots \ldots \ldots \ldots \ldots$ & 93.05 & 0.479 & 0.050 & $\ldots$ & $\ldots$ & 2.91 & 1.96 & 5.75 & $\ldots$ & $\ldots$ \\
\hline $\mathrm{J} 122927.1+752037 \ldots \ldots \ldots \ldots \ldots \ldots$ & 878.57 & 9.168 & 0.309 & $\ldots$ & $\ldots$ & 52.98 & 1.70 & 5.32 & $\ldots$ & $\ldots$ \\
\hline J122940.6+752106 .................. & 815.18 & 8.592 & 0.301 & $\ldots$ & $\ldots$ & 23.87 & 1.61 & 5.16 & $\ldots$ & $\ldots$ \\
\hline $\mathrm{J} 131709.9+285513 \ldots \ldots \ldots \ldots \ldots \ldots$ & 301.90 & 2.347 & 0.135 & $\ldots$ & $\ldots$ & 16.61 & 13.11 & 15.10 & $\ldots$ & $\ldots$ \\
\hline $\mathrm{J} 131722.0+285353 \ldots \ldots \ldots \ldots \ldots \ldots$ & 586.09 & 4.836 & 0.200 & 0.435 & 0.018 & 25.27 & 15.80 & 16.57 & 0.0612 & $\ldots$ \\
\hline $\mathrm{J} 134507.8+000359 \ldots \ldots \ldots \ldots \ldots \ldots$ & 43.10 & 3.733 & 0.568 & 16.615 & 2.531 & 12.97 & 7.35 & 11.29 & 0.3616 & $\ldots$ \\
\hline $\mathrm{J} 134514.6-000846 \ldots \ldots \ldots \ldots \ldots \ldots$ & 292.62 & 25.966 & 1.518 & 9.364 & 0.547 & 62.68 & 12.21 & 14.56 & 0.1179 & $\ldots$ \\
\hline $\mathrm{J} 141152.6+520937 \ldots \ldots \ldots \ldots \ldots \ldots$ & 62.79 & 0.504 & 0.064 & $\ldots$ & $\ldots$ & 9.78 & 2.04 & 5.87 & $\ldots$ & $\ldots$ \\
\hline J141556.8+230727 ................. & 160.64 & 8.851 & 0.698 & 31.138 & 2.457 & 11.81 & 5.46 & 9.72 & $\ldots$ & 0.327 \\
\hline $\mathrm{J} 141602.1+230647 \ldots \ldots \ldots \ldots \ldots \ldots \ldots$ & 94.96 & 5.211 & 0.535 & 34.307 & 3.520 & 33.59 & 5.07 & 9.37 & $\ldots$ & 0.427 \\
\hline $\mathrm{J} 153259.2-004414 \ldots \ldots \ldots \ldots \ldots \ldots \ldots$ & 708.42 & 69.601 & 2.615 & $\ldots$ & $\ldots$ & 87.89 & 1.61 & 5.16 & $\ldots$ & $\ldots$ \\
\hline $\mathrm{J} 153415.0+232459 \ldots \ldots \ldots \ldots \ldots \ldots$ & 150.02 & 1.906 & 0.156 & $\ldots$ & $\ldots$ & 6.63 & 6.14 & 10.32 & $\ldots$ & $\ldots$ \\
\hline $\mathrm{J} 154932.0+213300 \ldots \ldots \ldots \ldots \ldots \ldots$ & 335.87 & 5.945 & 0.324 & 80.582 & 4.397 & 11.71 & 4.06 & 8.37 & $\ldots$ & 0.577 \\
\hline $\mathrm{J} 160847.1+654139 \ldots \ldots \ldots \ldots \ldots \ldots$ & 202.61 & 4.912 & 0.345 & $\ldots$ & $\ldots$ & 26.57 & 14.22 & 15.72 & $\ldots$ & $\ldots$ \\
\hline $\mathrm{J} 160948.4+660056 \ldots \ldots \ldots \ldots \ldots \ldots$ & 378.22 & 4.414 & 0.227 & 48.071 & 2.472 & 22.04 & 1.32 & 4.61 & $\ldots$ & 0.527 \\
\hline J165514.4-082944 _................ & 47.76 & 4.039 & 0.584 & $\ldots$ & $\ldots$ & 22.08 & 3.15 & 7.35 & $\ldots$ & $\ldots$ \\
\hline J205537.4-043334 _................ & 237.63 & 4.098 & 0.266 & $\ldots$ & $\ldots$ & 7.28 & 6.13 & 10.31 & $\ldots$ & $\ldots$ \\
\hline J205617.2-044154 ................. & 88.51 & 1.464 & 0.156 & 21.846 & 2.322 & 3.09 & 3.18 & 7.39 & $0.6002^{\mathrm{h}}$ & 0.702 \\
\hline $\mathrm{J} 220455.8-181524 \ldots \ldots \ldots \ldots \ldots \ldots$ & 199.35 & 34.218 & 2.424 & $\ldots$ & $\ldots$ & 50.83 & 10.16 & 13.29 & $\ldots$ & $\ldots$ \\
\hline J221326.2-220532 …............. & 72.22 & 2.290 & 0.269 & 0.017 & 0.002 & 9.54 & 2.44 & 6.45 & $0.0180^{\mathrm{h}}$ & $\ldots$ \\
\hline $\mathrm{J} 223538.4+340609 \ldots \ldots \ldots \ldots \ldots \ldots$ & 149.88 & 5.406 & 0.442 & 26.510 & 2.165 & 3.58 & 3.52 & 7.78 & $0.3768^{\mathrm{h}}$ & $\ldots$ \\
\hline $\mathrm{J} 223614.5+335648 \ldots \ldots \ldots \ldots \ldots \ldots$ & 117.73 & 2.671 & 0.246 & $\ldots$ & $\ldots$ & 15.15 & 1.19 & 4.33 & $\ldots$ & $\ldots$ \\
\hline $\mathrm{J} 230150.7+084352 \ldots \ldots \ldots \ldots \ldots \ldots \ldots$ & 321.47 & 2.716 & 0.151 & 16.420 & 0.916 & 22.85 & 11.40 & 14.07 & $0.4118^{\mathrm{h}}$ & $\ldots$ \\
\hline J230227.7+083901 ...................... & 97.82 & 0.677 & 0.068 & 4.427 & 0.448 & 7.85 & 3.62 & 7.90 & $0.4256^{\mathrm{h}}$ & $\ldots$ \\
\hline $\mathrm{J} 230252.0+084137 \ldots \ldots \ldots \ldots \ldots \ldots$ & 456.11 & 2.874 & 0.134 & 0.116 & 0.005 & 6.44 & 0.98 & 3.82 & $0.0415^{\mathrm{h}}$ & $\ldots$ \\
\hline $\mathrm{J} 230311.1+085131 \ldots \ldots \ldots \ldots \ldots \ldots \ldots$ & 685.24 & 4.813 & 0.184 & $\ldots$ & $\ldots$ & 38.75 & 4.27 & 8.58 & $\ldots$ & $\ldots$ \\
\hline $\mathrm{J} 234817.8+010617 \ldots \ldots \ldots \ldots \ldots \ldots \ldots$ & 384.19 & 4.844 & 0.247 & 1.057 & 0.054 & 15.62 & 3.42 & 7.68 & $0.0932^{\mathrm{h}}$ & $\ldots$ \\
\hline
\end{tabular}

a All tabulated uncertainties are $1 \sigma$ values.

${ }^{\mathrm{b}}$ Luminosities are calculated from spectroscopic redshifts unless only VTP estimates are available.

c Source core radius is estimated from the circular $\beta$ model fits. The uncertainty in $\Delta r_{c}$ is on the order of $10 \%-20 \%$.

d Chandra PSF size at the location of extended X-ray source. The PSF is derived from the best-fit analytic relation between the Gaussian sigma of point sources and is similar to the radius encircling $50 \%$ of total counts for a monochromatic source at $0.75 \mathrm{keV}$.

e Off-axis angle.

${ }^{\mathrm{f}}$ Redshifts obtained from the literature have NED IDs listed in Table 5. Those marked by a superscript $h$ are from ChaMP spectroscopy. Typical spectroscopic redshift uncertainties are $\sim 0.0006$

${ }^{\mathrm{g}}$ Red-sequence filtered VTP corrected redshifts. The dispersion of the VTP redshifts about the spectroscopic values is 0.03 .

h Redshifts measured from our ChaMP spectroscopic program. 


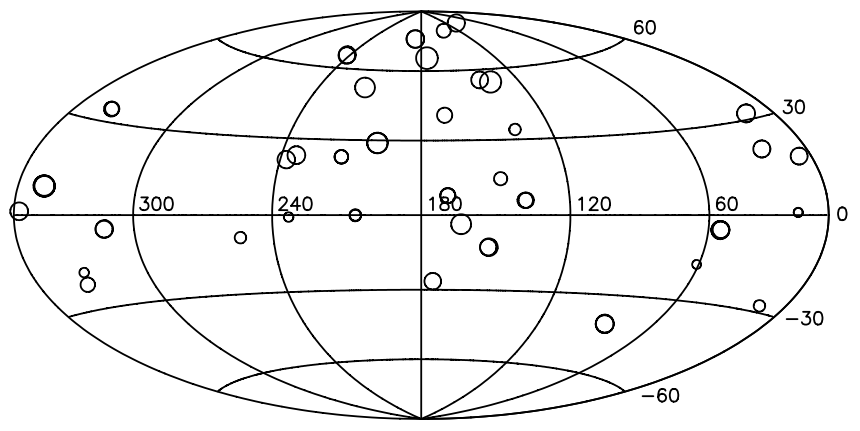

FIG. 2.-Aitoff equatorial projection in Galactic coordinates of 55 extended $\mathrm{X}$-ray sources. Symbol size is proportional to X-ray exposure time (see Table 1).

the color-magnitude plane allows our galaxy color slices to overlap with adjacent regions by $\sim 0.1 \mathrm{mag}$ in $r^{\prime}-i^{\prime}$. Thus, we completely cover the color-magnitude plane with overlapping color slices in the region expected for our cluster redshift range. The overlapping color slices prevent us from missing clusters whose red sequence may fall between our adopted 27 red sequence (i.e., redshift) models.

In addition to selecting galaxies by color, we also restrict the magnitude range for each color slice to enhance the cluster signature above the background. We have elected to include galaxies with $i^{\prime}$-band magnitudes in the range of $m_{i^{\prime}}^{*}-3$ to $m_{i^{\prime}}^{*}+4$, where $m_{i^{\prime}}^{*}$ is the apparent magnitude of the turnover in the Schechter function representation of the cluster luminosity function (Schechter 1976). The $m_{i^{\prime}}^{*}$ value was derived by taking the value of $M_{R_{c}}^{*}=-22.20+5 \log h_{50}$ from Barkhouse (2003) for a sample of 57 low-redshift clusters and transforming to $M_{i^{\prime}}^{*}=$ $-21.52+5 \log h_{70}$ using the equations of Frei \& Gunn (1994). For each redshift interval, we transformed $M_{i^{\prime}}^{*}$ into $m_{i^{\prime}}^{*}$ using the appropriate luminosity distance and $K$-correction tabulated in Frei \& Gunn (1994) for an early-type population (no correction

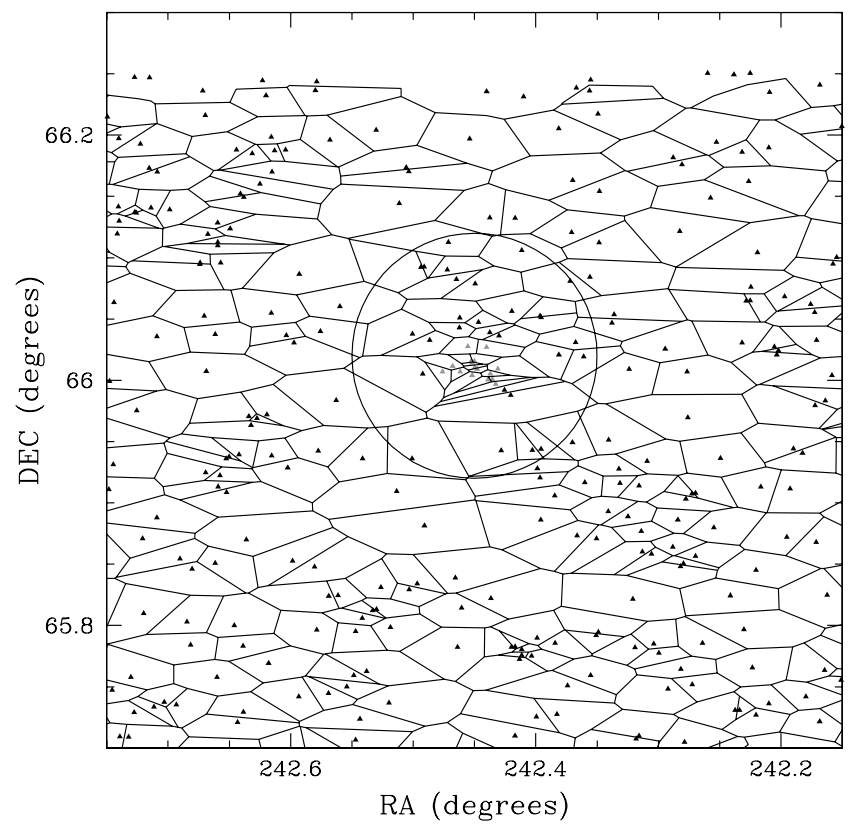

FIG. 3.-Voronoi tessellation on the galaxy distribution for ObsID 2127. Only galaxies satisfying the color cut expected for a cluster red sequence at a redshift of 0.475 are depicted. The area enclosed within the circle is a previously unknown cluster at an estimated VTP redshift of 0.527 . This cluster was also detected as an extended X-ray source and is listed as object CXOMP $\mathrm{J} 160948.4+660057$ in our data tables.

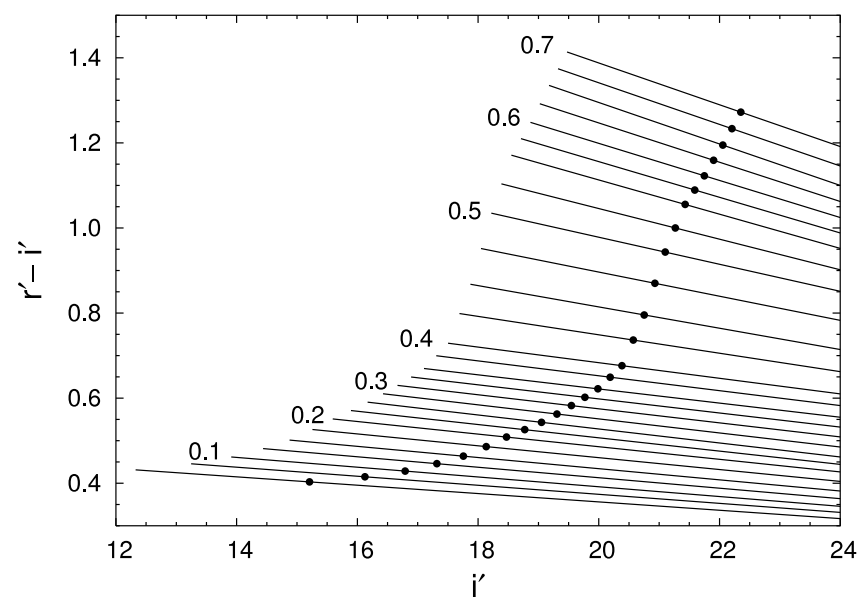

FIG. 4.- Red-sequence model color slices used to select galaxies at various redshifts (indicated to the left of the lines) as part of the cluster detection process using VTP. Galaxy catalogs are constructed for each red sequence by selecting galaxies with $r^{\prime}-i^{\prime}$ color within \pm 0.1 mag of the red-sequence line. Overlapping color slices allow us to completely sample the color-magnitude plane for $z=0.05-0.70$. The filled circles depict the $i^{\prime}$-band magnitude of $m^{*}$ for our sampled redshift range.

for galaxy evolution was applied). We note that galaxies can be included using a smaller magnitude range if CCD image saturation occurs at a magnitude fainter than $m_{i^{\prime}}^{*}-3$ or if the magnitude limit (measured as the turnover in the galaxy counts) is brighter than $m_{i^{\prime}}^{*}+4$ (see Table 2 for the turnover magnitude limits). Our magnitude restriction thus ensures that galaxies are included in a consistent fashion for each red sequence (i.e., redshift) slice, while excluding bias due to saturation and incompleteness effects.

The final culling we conducted on our galaxy catalogs was to exclude all objects with a stellarity class $>0.9$ in the $i^{\prime}$ band (i.e., "starlike" objects), as output by SExtractor (Bertin \& Arnouts 1996). The median seeing of our optical images is 1 ."0, which corresponds to a linear distance of $\sim 7 \mathrm{kpc}$ at $z=0.7$ for our adopted cosmology. The stellarity cut thus enabled us to produce a statistically high fidelity sample of galaxies, facilitating redsequence detection.

Once galaxy catalogs for our 27 red-sequence slices had been compiled for each field, we ran VTP independently on our 56 mosaic fields. For this paper we restrict the analysis and discussions to a sample of 36 mosaic fields (see Table 2), which contain VTPdetected optical cluster candidates located within the field of view of the Chandra observer identifications (ObsIDs; total sky coverage of $6.1 \mathrm{deg}^{2}$ ). This allows us to fairly compare cluster detection fractions and measure optical and X-ray properties of matched, serendipitously detected, extended sources.

\subsubsection{Monte Carlo Simulations}

In addition to supplying galaxy catalogs, it is necessary to set the detection threshold and rejection probability limit against random background fluctuations as inputs to VTP. In Ramella et al. (2001) the detection threshold was set at the $80 \%$ confidence level, and detected sources with a probability $>5 \%$ of being random background fluctuations were rejected from the final cluster compilation.

To determine the best choice of detection and rejection parameters, we conducted a series of extensive Monte Carlo simulations. We adopted the contamination rate as a benchmark to compare different runs of VTP. The contamination rate is defined as $C=N_{S} / N_{D}$, where $N_{S}$ is the number of detected clusters from a simulated galaxy catalog, and $N_{D}$ is the number of detected clusters based on the original catalog using the same VTP input 
parameters as $N_{S}$. The goal of the simulations is to run VTP for a range in values of the threshold and rejection probability to determine which values minimize the contamination rate while maximizing the detection of real clusters.

The simulated cluster catalogs were constructed using three different procedures to alter the original galaxy catalog generated by SExtractor for each mosaic field, including randomizing positions and shuffling magnitudes and colors. For the first procedure we randomized galaxy positions while keeping the magnitudes and colors the same. Next 1000 simulated galaxy catalogs were constructed for each set of threshold and rejection-probability pairs (30 combinations in total were used). Each of the 1000 simulated catalogs was divided into 27 red-sequence slices (as described in $\S 3.2 .1)$ and VTP was run separately on each. The average contamination rate, $C_{\text {ave }}$, was calculated for each set of 1000 simulated catalogs for each ordered pair of the detection threshold and random probability values. In total, VTP was run on 30,000 simulated galaxy fields, each containing 27 red-sequence slices (i.e., 810,000 executions of the VTP algorithm). The minimum value of the contamination rate was found to be $C_{\text {ave }}=12.8 \%$ for a detection threshold value of $90 \%$ confidence level and a random probability limit of 5\% (i.e., overdensities having a probability $>5 \%$ of being a random background fluctuation are rejected). Note that these values are very similar to those used in Ramella et al. (2001).

The second procedure we used to measure the false-positive rate involved shuffling (without replacement) the $i^{\prime}$-band magnitudes while maintaining the original galaxy colors and positions. The magnitudes are shuffled rather than assigned randomly to preserve the galaxy brightness distribution as measured for the real data. The simulations were undertaken in the same manner as conducted previously for the random galaxy position catalogs. The minimum contamination rate for the shuffled magnitude catalogs was determined to be $C_{\mathrm{ave}}=20.76 \%$. This minimum value coincided with the same pair of VTP detection parameters as that found above using the randomized position catalogs (i.e., $90 \%$ confidence level and a random probability threshold of 5\%).

For the final test galaxy colors were shuffled while retaining the original magnitudes and positions. Using the same number of simulations as described above, the minimum contamination rate was found to be $C_{\text {ave }}=7.52 \%$. This minimum value coincidently occurred for the same pair of VTP detection parameters as found for the previous two independent sets of simulations. Thus, our Monte Carlo simulations demonstrate that executing VTP with a confidence level of $90 \%$ and a random probability limit of $5 \%$ will minimize the contamination rate.

The false-positive tests show that shuffling the galaxy colors produces the smallest contamination rate, while shuffling the $i^{\prime}$-band magnitudes exhibits the largest. This is directly related to the red-sequence slices that we use prior to running the VTP detection. Since cluster red sequences based on $r^{\prime}-i^{\prime}$ versus $i^{\prime}$ are approximately horizontal for a wide range in redshift (see Fig. 4), shuffling galaxy $i^{\prime}$ magnitudes while maintaining their original color has the least effect on washing out the signature of real clusters in the simulated catalogs. Conversely, shuffling colors is expected to have the greatest impact since the red sequence of real clusters will be smoothed out. Although our simulations were not designed to reproduce the two-point correlation function for field galaxies (which would be expected to have a higher contamination rate than a randomized position catalog; Gilbank et al. 2004), the simulations produced by shuffling the colors should be a reasonable estimate of the contamination rate. Thus, we surmise that our red-sequence VTP method suffers from a contamination rate of $<20 \%$.
It is important to note that our simulations are not designed to provide a perfect measure of the false-positive rate, but rather serve as an indicator on how to tune our cluster detection code. We therefore adopt a detection threshold of a $90 \%$ confidence level and a cutoff probability for a random fluctuation of 5\% for our red-sequence VTP application.

\subsubsection{Final Optically Selected Cluster Sample}

Once the VTP cluster detection was implemented for the 27 red-sequence slices per field with the input parameters derived from our Monte Carlo simulations, we merged our candidate clusters into a single catalog. The redshift assigned to each cluster is determined by selecting the red-sequence slice that maximized the product of the confidence level and contrast above background. These parameters are output by the VTP algorithm and are not the same as the confidence threshold and random fluctuation level set as input to VTP (Ramella et al. 2001). As an additional step to minimize spurious sources, we include only those detections that have an output-measured confidence level $>99 \%$ (this value was selected by visually inspecting the VTP source catalog). This culling procedure was also implemented for the simulations described in $\S 3.2 .2$. For cluster centroids that are $<2^{\prime}$ apart and separated by $\Delta z<0.1$ (estimated from the specific red-sequence slice from which the cluster was detected with maximum probability), we merged the candidates into a single cluster. Finally, we visually inspected our cluster candidates to exclude spurious or contaminated clusters/groups from the final sample.

\section{RESULTS}

With the construction of two independent cluster compilations derived from the same sky area, we are able to compare and contrast the attributes of our two samples, each selected from different wavelength regimes. In this section we describe the properties of each cluster sample and compare cluster detection techniques.

\subsection{Optical Cluster Properties}

Our final sample of optical cluster candidates selected using our red-sequence VTP technique-independent of X-ray detections - contains 115 sources measured from 36 mosaic optical fields. As mentioned previously, this sample contains only optical VTP detections from regions that overlap with the Chandra sky coverage and excludes Chandra PI targets (of which 15 clusters are detected by our VTP method).

A main benefit of using VTP on galaxies selected relative to the red sequence is that we are able to assign a photometric redshift to each cluster candidate. Since a perfect match between the filter transmission function used to obtain our mosaic data and that assumed for the red-sequence models is not expected, estimated red-sequence redshifts may be systematically offset from the "true" values. To quantify this effect, we compared the photometric redshifts estimated from VTP with spectroscopically determined redshifts for a sample of 15 known clusters $(0.3 \leq$ $z \leq 0.7)$ contained in our ChaMP mosaic fields. These clusters are not included in our final cluster sample, since they are either Chandra PI targets or not found within the spatial coverage of our 36 optical ChaMP fields that overlap our X-ray sky coverage. In Figure 5 we plot spectroscopic redshifts versus VTP redshifts for the 15 cluster sample, which illustrates a systematic offset in the VTP redshifts relative to the spectroscopic values in the sense that the photometrically derived cluster redshifts are underestimated. A line of slope unity yields a good fit to the data, with an offset of 


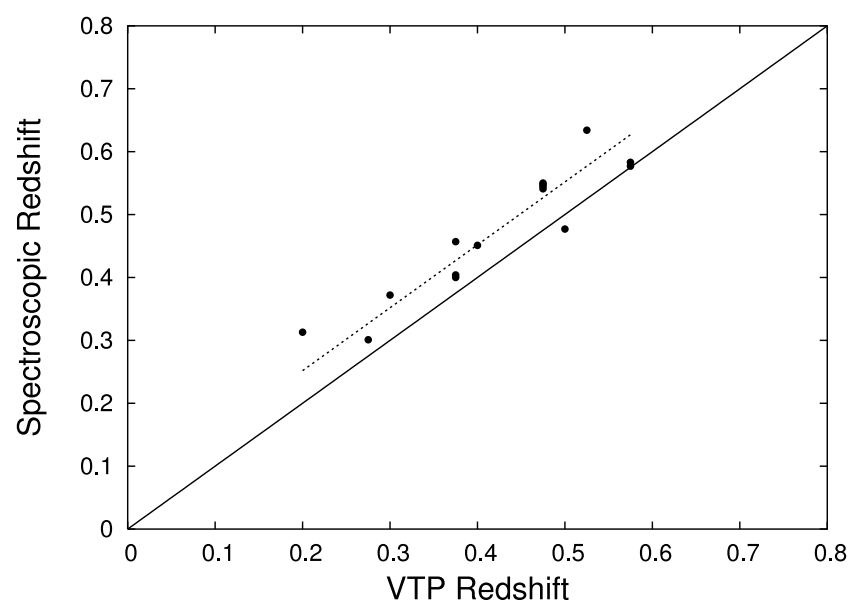

FIG. 5.-Comparison between spectroscopic and VTP derived redshifts for a sample of 15 previously known rich clusters $(0.3 \leq z \leq 0.7)$ within our sampled fields. The dashed line represents a fit to the offset between the two redshift measurements $(\Delta z=+0.052)$.

$\Delta z=+0.052$ and a dispersion of 0.033 (Fig. 5, dashed line). To improve VTP redshift estimates for our cluster sample, we add the correction term of $\Delta z=0.052$ to all photometrically derived redshifts. For X-ray-detected clusters, the VTP redshift is included in Table 3. Table 4 lists the cluster candidates detected solely from the application of the red-sequence-filtered VTP on the 36 mosaic fields that overlap our Chandra fields tabulated in Table 1. Corrected VTP redshifts and limits to $f_{\mathrm{X}}$ and $L_{\mathrm{X}}$ are also provided there.

In Figure 6 we present a comparison between seven ChaMP serendipitously detected extended X-ray sources that are matched in the optical and have both spectroscopic and VTP estimated redshifts. The open circles are for VTP derived redshifts without the $\Delta z$ correction applied, while the solid circles include the correction. The application of the redshift correction lowers the dispersion of the VTP-estimated redshifts from the corresponding spectroscopic measurements from 0.05 to 0.03 .

\subsection{X-Ray Cluster Properties}

The availability of multiwavelength archival data affords the opportunity to conduct a survey for specific objects with minimal investment in observing time. In Figure 7 we show the distribution of X-ray flux in the $0.5-2 \mathrm{keV}$ energy band for our 55 extended sources as a function of vignetting-corrected exposure time. The median flux of the sample is $f_{\mathrm{X}}=(4.84 \pm 0.15) \times$ $10^{-14} \mathrm{ergs} \mathrm{s}^{-1} \mathrm{~cm}^{-2}$ with $\sim 82 \%$ of the sources detected in exposures $<50 \mathrm{ks}$.

A histogram of the redshift distribution for 31 of our X-ray sources with redshifts is presented in Figure 8. Redshift values are measured either spectroscopically from ChaMP (or other published sources; see Table 5) or from our red-sequence-filtered VTP technique. Six sources at $z<0.1$ are coincident with nearby single galaxies, as ascertained by examining their positions in our mosaic data (Kim et al. 2006). The final extended X-ray source catalog thus contains 49 clusters and 6 low- $z$ galaxies. The average redshift of the cluster-only sample is $\bar{z}=0.41$, which corresponds to the peak in the redshift histogram distribution.

The distribution of the X-ray luminosity $(0.5-2 \mathrm{keV})$ for our 31 sources with estimated redshifts is depicted in Figure 9. The $\mathrm{X}$-ray luminosity spans the range from rich clusters $\left(L_{\mathrm{X}} \sim 10^{43}-\right.$ $10^{45}$ ergs s$^{-1}$; Rosati et al. 2002) to poor groups $\left(L_{\mathrm{X}} \sim 10^{41}\right.$ $10^{43} \mathrm{ergs} \mathrm{s}^{-1}$; Mulchaey 2000) and bright starburst galaxies
( $L_{\mathrm{X}} \gtrsim 10^{39} \mathrm{ergs} \mathrm{s}^{-1}$; Fabbiano 1989). The median value of the luminosity for the complete sample of extended sources is $L_{\mathrm{X}}=$ $(2.18 \pm 0.18) \times 10^{43} \mathrm{ergs} \mathrm{s}^{-1}$, which increases to $L_{X}=(2.65 \pm$ $0.19) \times 10^{43} \mathrm{ergs} \mathrm{s}^{-1}$ when the six low- $z$ galaxies are excluded.

In $\S 4.3$ we compare the cluster candidates detected by our redsequence VTP method with the extended X-rays sources in the sky area where our X-ray and optical imaging overlaps (6.1 deg 2 ). In several instances the X-ray counterpart to the optical detection is absent. For these cases we compute the upper limit X-ray flux by measuring photon counts in a $84^{\prime \prime}$ aperture radius centered on the optical cluster position as defined by the location of the brightest cluster galaxy (BCG) or the centroid position output by the VTP algorithm. The $3 \sigma$ upper limits are computed by extrapolating the counts to infinity using the $\beta$ model (see $\S 3.1$ ) and then converting to luminosity limits using the redshift estimated by the VTP process, or if available, that derived spectroscopically from ChaMP or other published sources. The $84^{\prime \prime}$ radius represents a factor of 4 times the median core radius of the 55 extended X-ray sources and is equivalent to a core radius of $460 \mathrm{kpc}$ at $z=0.41$ (median redshift of the cluster-only sample) for our adopted cosmology. The X-ray flux and luminosity upper limits, for the $0.5-2 \mathrm{keV}$ energy band, are tabulated in Table 4 for the 102 clusters with optical-only detections.

In Figure 9 the X-ray luminosity distribution of the upper limits (dashed line) is compared to the distribution of the detected $\mathrm{X}$-ray sources. From this figure it appears that many of the optical sources not detected in the X-rays have luminosities characteristic of groups and clusters (median $L_{X} \sim 10^{43} \operatorname{ergs~s}^{-1}$ ), thus making them harder to detect in the shallower X-ray fields at the expected faint flux levels based on the $L_{\mathrm{X}}-T_{\mathrm{X}}$ and $M_{\mathrm{tot}}-T_{\mathrm{X}}$ relations (e.g., Ettori et al. 2004). The distribution of X-ray luminosity with redshift is depicted in Figure 10. The circles represent the sample of $25 \mathrm{X}$-ray-detected clusters with estimated redshifts, while the triangles depict the six extended X-rays sources identified with low- $z$ single galaxies. Also plotted are the X-ray luminosity upper limits (arrows) for sources detected only in the optical by VTP. Sources with spectroscopic redshifts are shown as the larger symbols while objects with only VTP estimated redshifts are marked with the smaller symbols. Even though the X-ray flux limit varies from field to field due to the wide range in exposure times (see Fig. 7), the solid line in Figure 10 indicates the luminosity for a flux limit of $f_{\mathrm{X}}(0.5-2 \mathrm{keV})=1.5 \times$ $10^{-14} \mathrm{ergs} \mathrm{s}^{-1} \mathrm{~cm}^{-2}$. This flux limit is plotted for comparison purposes only, but it is a reasonable estimate of the overall flux limit of the survey.

To compare the ChaMP sources with previous surveys, we plot in Figure 10 the 447 clusters (plus signs) from the ROSATESO Flux-Limited X-Ray (REFLEX) Galaxy Cluster Survey (Böhringer et al. 2004). The luminosity range probed by our sample is at least an order of magnitude below that of previous largearea ROSAT samples like REFLEX. This provides newly extended coverage of the $L_{\mathrm{X}}$-redshift plane down to group luminosities even at significant look-back times, thus paving the way for studies of cluster evolution that can take into account not just cosmic time but also mass/luminosity/temperature effects. In addition to the REFLEX cluster survey, we also plot 200 galaxy clusters (crosses) from the 160 Square Degree ROSAT Survey (Mullis et al. 2003). This cluster sample is well matched to our ChaMP sample in terms of the measured range in luminosity and redshift. The median redshift of the 160 Square Degree survey clusters is $z=0.25$, which is lower than the median redshift of the ChaMP cluster-only sample $(z=0.41)$.

The core radius for each extended X-ray source is measured with $\beta=0.67$ due to the small number of photons expected for 
TABLE 4

VTP Clusters with X-RAy Upper Limits

\begin{tabular}{|c|c|c|c|c|c|c|c|}
\hline No. & ObsID & $\begin{array}{c}\text { R.A. } \\
\text { (J2000.0) }\end{array}$ & $\begin{array}{c}\text { Decl. } \\
\text { (J2000.0) }\end{array}$ & Redshift $^{\mathrm{a}}$ & $\begin{array}{c}f_{\mathrm{X}}^{\mathrm{b}} \\
\left(10^{-14} \mathrm{cgs}\right)\end{array}$ & $\begin{array}{c}L_{\mathrm{X}} \\
\left(10^{42} \mathrm{cgs}\right)\end{array}$ & $\begin{array}{c}\mathrm{PSF}^{\mathrm{c}} \\
(\operatorname{arcsec})\end{array}$ \\
\hline 1 & 2099 & 002359.1 & -015017.0 & 0.127 & $\leq 2.947$ & $\leq 1.256$ & 2.52 \\
\hline 2 & 521 & 010127.6 & +314646.9 & 0.227 & $\leq 1.167$ & $\leq 1.795$ & 3.47 \\
\hline 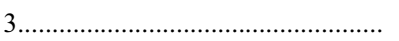 & 521 & 010131.0 & +314644.4 & 0.402 & $\leq 1.207$ & $\leq 6.942$ & 2.88 \\
\hline 4 & 521 & 010154.7 & +314538.1 & 0.502 & $\leq 1.181$ & $\leq 11.518$ & 0.73 \\
\hline 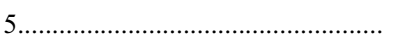 & 521 & 010208.7 & +315555.0 & 0.577 & $\leq 1.166$ & $\leq 15.901$ & 3.67 \\
\hline 6.1.1. & 813 & 010253.8 & -270722.8 & $0.221^{\mathrm{d}}$ & $\leq 3.664$ & $\leq 5.321$ & 12.37 \\
\hline 7 & 913 & 015244.9 & -140132.6 & 0.527 & $\leq 1.244$ & $\leq 13.640$ & 1.72 \\
\hline 8 & 913 & 015316.0 & -135718.0 & 0.577 & $\leq 1.269$ & $\leq 17.309$ & 2.53 \\
\hline 9 & 913 & 015318.1 & -135200.4 & 0.177 & $\leq 1.303$ & $\leq 1.149$ & 3.91 \\
\hline 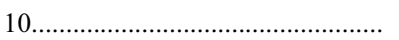 & 796 & 033641.7 & -045352.0 & 0.352 & $\leq 2.022$ & $\leq 8.514$ & 18.13 \\
\hline $11 \ldots \ldots \ldots$ & 796 & 033642.4 & -045930.4 & 0.727 & $\leq 2.238$ & $\leq \overline{53} .553$ & 14.19 \\
\hline 12 & 796 & 033731.8 & -051021.7 & 0.202 & $\leq 1.070$ & $\leq 1.266$ & 4.32 \\
\hline 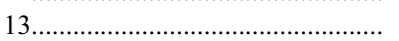 & 796 & 033801.7 & -045343.9 & 0.527 & $\leq 1.133$ & $\leq 12.420$ & 5.32 \\
\hline 14 & 914 & 054138.7 & $\begin{array}{lll}-41 & 10 & 09.2\end{array}$ & 0.527 & $\leq 1.142$ & $\leq 12.524$ & 17.50 \\
\hline 15 & 914 & 054224.9 & -410014.2 & 0.502 & $\leq 1.114$ & $\leq 10.866$ & 1.40 \\
\hline 16 & 914 & 054225.1 & -405312.9 & 0.577 & $\leq 0.914$ & $\leq 12.469$ & 2.99 \\
\hline 17 & 914 & 054236.3 & -405007.6 & 0.527 & $\leq 0.939$ & $\leq 10.296$ & 4.67 \\
\hline 18. & 914 & 054257.5 & -405808.2 & 0.527 & $<0.930$ & $<10.200$ & 0.53 \\
\hline 19 & 1602 & 062459.1 & +815909.6 & 0.252 & $\leq 0.981$ & $\leq 1.911$ & 1.23 \\
\hline 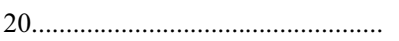 & 926 & 100928.8 & -124434.8 & 0.402 & $\leq 0.999$ & $\leq 5.746$ & 7.92 \\
\hline 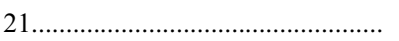 & 926 & 100935.8 & -124354.4 & 0.602 & $\leq 1.344$ & $\leq 20.314$ & 5.66 \\
\hline 22 & 926 & 100939.9 & -124554.6 & 0.277 & $\leq 1.219$ & $\leq 2.947$ & 5.50 \\
\hline 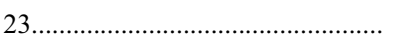 & 926 & 100948.1 & -123849.5 & 0.352 & $\leq 1.222$ & $\leq 5.145$ & 2.75 \\
\hline 24 & 926 & 101105.7 & -124030.5 & 0.277 & $\leq 1.446$ & $\leq 3.497$ & 8.94 \\
\hline 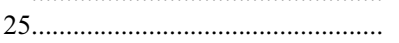 & 512 & 105648.9 & -033725.5 & $0.182^{\mathrm{e}}$ & $\leq 0.902$ & $\leq 0.846$ & 0.63 \\
\hline 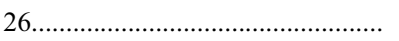 & 512 & 105700.5 & -034419.6 & 0.277 & $\leq 0.692$ & $\leq 1.674$ & 1.60 \\
\hline 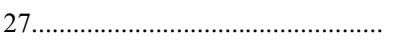 & 915 & 111344.2 & -262338.4 & 0.627 & $\leq 1.616$ & $\leq 26.959$ & 10.91 \\
\hline 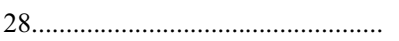 & 363 & 111720.4 & +075856.3 & 0.577 & $\leq 1.961$ & $\leq 26.753$ & 21.31 \\
\hline 29 & 363 & 111735.7 & +074252.1 & 0.527 & $\leq 1.490$ & $\leq 16.330$ & 5.86 \\
\hline 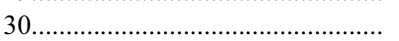 & 363 & 111741.8 & +074503.6 & 0.402 & $\leq 1.348$ & $\leq 7.753$ & 3.98 \\
\hline 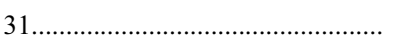 & 363 & 111816.6 & +074323.9 & 0.277 & $\leq 2.550$ & $\leq 6.168$ & 0.54 \\
\hline 32 & 898 & 113950.4 & -263423.3 & 0.702 & $\leq 2.043$ & $\leq 44.869$ & 9.85 \\
\hline 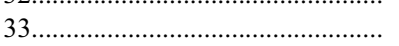 & 898 & 114040.4 & -263402.8 & 0.577 & $\leq 1.640$ & $\leq 22.368$ & 1.01 \\
\hline 34 & 898 & 114046.8 & -263444.4 & 0.452 & $\leq 1.136$ & $\leq 8.625$ & 1.22 \\
\hline 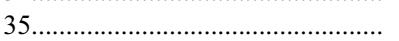 & 898 & 114052.2 & -262407.3 & 0.427 & $\leq 2.293$ & $\leq 15.208$ & 2.38 \\
\hline 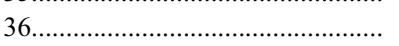 & 2210 & 125621.3 & +4715 55.7 & $0.209^{\mathrm{d}}$ & $\leq 1.089$ & $\leq 1.390$ & 3.21 \\
\hline 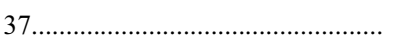 & 2210 & 125644.1 & +471843.6 & 0.577 & $\leq 1.696$ & $\leq 23.133$ & 0.65 \\
\hline 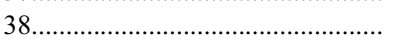 & 2228 & 131640.7 & +290623.3 & 0.652 & $\leq 0.606$ & $\leq 11.110$ & 3.63 \\
\hline 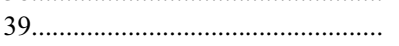 & 2228 & 131654.0 & +291420.8 & 0.477 & $\leq 0.641$ & $\leq 5.532$ & 1.90 \\
\hline 40 & 2228 & 131721.1 & +292042.0 & 0.652 & $\leq 0.630$ & $\leq 11.552$ & 6.44 \\
\hline 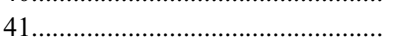 & 2228 & 131722.8 & +285848.0 & 0.377 & $\leq 0.704$ & $\leq 3.483$ & 7.95 \\
\hline 42 & 507 & 134718.5 & -115226.7 & 0.402 & $<2.476$ & $<14.241$ & 2.54 \\
\hline 43 & 507 & 134727.7 & -114038.9 & $0.086^{\mathrm{d}}$ & $\leq 3.428$ & $\leq 0.641$ & 1.95 \\
\hline 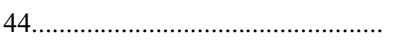 & 2024 & 141523.3 & +231152.1 & 0.552 & $\leq 2.201$ & $\leq 26.976$ & 12.95 \\
\hline 45 & 2024 & 141544.1 & +231425.4 & 0.252 & $\leq 2.534$ & $\leq 4.936$ & 5.84 \\
\hline 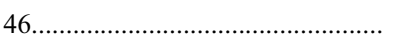 & 2024 & 141550.1 & +231358.5 & 0.352 & $\leq 2.373$ & $\leq 9.990$ & 4.37 \\
\hline 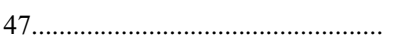 & 930 & 141550.9 & +113252.8 & 0.227 & $\leq 2.504$ & $\leq 3.850$ & 0.81 \\
\hline 48. & 930 & 141556.4 & +113014.4 & 0.527 & $\leq 2.645$ & $\leq 29.000$ & 0.74 \\
\hline 49 & 930 & 141603.2 & +112514.4 & 0.702 & $\leq 2.255$ & $\leq 49.541$ & 2.66 \\
\hline 50 & 541 & 141609.6 & +44 4402.4 & 0.427 & $\leq 1.462$ & $\leq 9.699$ & 3.37 \\
\hline $51 \ldots \ldots \ldots \ldots-1$. & 2024 & 141619.2 & +230559.3 & 0.577 & $\leq 1.806$ & $\leq \overline{2} 4.631$ & 3.99 \\
\hline 52 & 2024 & 141619.7 & +231958.8 & 0.752 & $\leq 2.630$ & $\leq 68.346$ & 2.28 \\
\hline 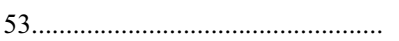 & 541 & 141627.6 & +445244.4 & 0.452 & $\leq 1.297$ & $\leq 9.851$ & 1.73 \\
\hline 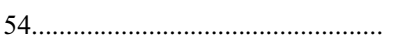 & 1657 & 142256.9 & +240827.6 & 0.227 & $\leq 1.828$ & $\leq 2.811$ & 6.46 \\
\hline 55 & 1657 & 142305.1 & +240024.0 & 0.502 & $\leq 1.826$ & $\leq 17.816$ & 3.26 \\
\hline 56 & 1657 & 142335.0 & +234948.2 & 0.502 & $\leq 2.128$ & $\leq 20.761$ & 9.76 \\
\hline 57 & 367 & 142355.9 & +230336.0 & 0.427 & $\leq 1.787$ & $\leq 11.853$ & 8.35 \\
\hline 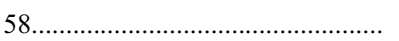 & 367 & 142437.0 & +2305 56.4 & 0.227 & $\leq 1.494$ & $\leq 2.298$ & 6.01 \\
\hline 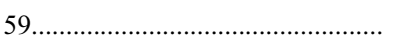 & 367 & 142443.0 & +2306 14.4 & 0.477 & $\leq 0.964$ & $\leq 8.322$ & 6.52 \\
\hline 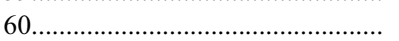 & 367 & 142504.6 & +225620.4 & 0.627 & $\leq 2.315$ & $\leq 38.627$ & 2.63 \\
\hline 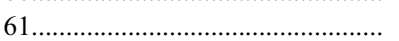 & 800 & 151343.2 & +364355.2 & 0.202 & $\leq 1.569$ & $\leq 1.856$ & 6.31 \\
\hline 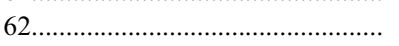 & 800 & 151429.3 & +364033.6 & 0.252 & $\leq 1.436$ & $\leq 2.798$ & 1.46 \\
\hline 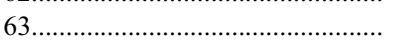 & 326 & 154850.9 & +212906.0 & 0.277 & $\leq 1.262$ & $\leq 3.052$ & 10.40 \\
\hline 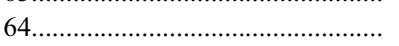 & 326 & 154901.3 & +213016.7 & 0.377 & $\leq 1.322$ & $\leq 6.534$ & 7.74 \\
\hline 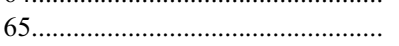 & 326 & 154905.1 & +212042.3 & 0.577 & $\leq 0.841$ & $\leq \overline{1} 1.474$ & 6.55 \\
\hline $66 \ldots$ & 326 & 154931.6 & +212336.7 & 0.502 & $<1.029$ & $<10.038$ & 0.98 \\
\hline
\end{tabular}


TABLE 4-Continued

\begin{tabular}{|c|c|c|c|c|c|c|c|}
\hline No. & ObsID & $\begin{array}{c}\text { R.A. } \\
(\mathrm{J} 2000.0)\end{array}$ & $\begin{array}{c}\text { Decl. } \\
(\mathrm{J} 2000.0)\end{array}$ & Redshift $^{\mathrm{a}}$ & $\begin{array}{c}f_{\mathrm{X}}^{\mathrm{b}} \\
\left(10^{-14} \mathrm{cgs}\right)\end{array}$ & $\begin{array}{c}L_{\mathrm{X}} \\
\left(10^{42} \mathrm{cgs}\right)\end{array}$ & $\begin{array}{c}\mathrm{PSF}^{\mathrm{c}} \\
(\operatorname{arcsec})\end{array}$ \\
\hline 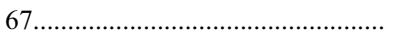 & 326 & 154941.8 & +212900.3 & 0.227 & $\leq 1.638$ & $\leq 2.519$ & 0.97 \\
\hline $68 \ldots$ & 2127 & 160810.1 & +654420.4 & 0.302 & $\leq 0.984$ & $\leq 2.904$ & 13.24 \\
\hline 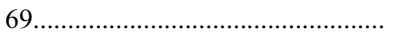 & 2127 & 160827.1 & +654622.8 & 0.477 & $\leq 1.303$ & $\leq 11.250$ & 8.98 \\
\hline (2) & 546 & 162233.3 & +263044.4 & 0.177 & $\leq 1.737$ & $\leq 1.532$ & 9.54 \\
\hline 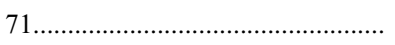 & 2221 & 171408.4 & +502056.4 & 0.627 & $\leq 0.932$ & $\leq 15.546$ & 2.08 \\
\hline 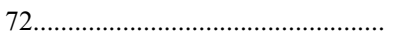 & 2221 & 171417.5 & +500256.4 & 0.527 & $\leq 1.077$ & $\leq 11.811$ & 8.58 \\
\hline 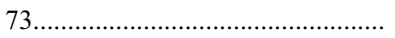 & 1899 & 180625.9 & +455452.3 & 0.627 & $\leq 1.253$ & $\leq 20.900$ & 7.25 \\
\hline 74 & 1899 & 180632.2 & +460054.0 & 0.677 & $\leq 1.372$ & $\leq 27.576$ & 10.53 \\
\hline 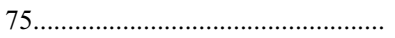 & 1899 & 180717.5 & +454702.4 & 0.252 & $\leq 0.978$ & $\leq 1.905$ & 1.30 \\
\hline 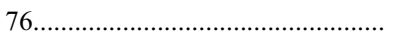 & 1899 & 180748.0 & +455649.2 & 0.677 & $\leq 0.941$ & $\leq 18.923$ & 2.56 \\
\hline 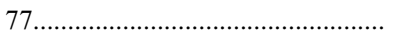 & 842 & 201048.2 & -485002.4 & 0.477 & $\leq 5.484$ & $\leq 47.342$ & 10.78 \\
\hline $78 \ldots \ldots \ldots \ldots \ldots \ldots \ldots$ & 551 & 205542.8 & -043355.7 & 0.277 & $\leq 0.970$ & $\leq 2.346$ & 4.63 \\
\hline $79 \ldots$. & 551 & 205652.0 & -043911.7 & 0.227 & $\leq 1.145$ & $\leq 1.760$ & 5.25 \\
\hline $80 \ldots \ldots \ldots \ldots \ldots \ldots$ & 928 & 213927.3 & -234226.9 & 0.677 & $\leq 1.211$ & $\leq 24.347$ & 6.72 \\
\hline $81 \ldots \ldots \ldots \ldots \ldots \ldots \ldots$ & 928 & 213930.5 & -233657.6 & 0.352 & $\leq 1.096$ & $\leq 4.613$ & 6.50 \\
\hline ............................. & 928 & 214024.5 & -234241.2 & 0.552 & $\leq 0.898$ & $\leq 11.013$ & 0.75 \\
\hline 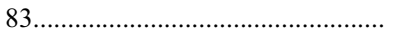 & 1644 & 215116.7 & -273450.9 & 0.327 & $\leq 2.830$ & $\leq 10.038$ & 7.70 \\
\hline 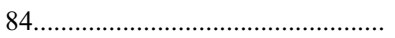 & 2113 & 215708.0 & -195127.1 & 0.352 & $\leq 4.629$ & $\leq 19.488$ & 0.50 \\
\hline 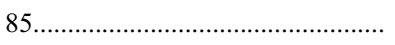 & 2114 & 220452.8 & -181536.2 & 0.277 & $\leq 8.549$ & $\leq 20.673$ & 9.08 \\
\hline $86 \ldots \ldots \ldots \ldots \ldots \ldots$ & 1479 & 221253.5 & -220939.0 & 0.202 & $\leq 1.644$ & $\leq 1.945$ & 1.24 \\
\hline 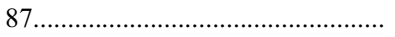 & 1479 & 221255.7 & -221210.5 & 0.652 & $\leq 1.602$ & $\leq 29.390$ & 1.00 \\
\hline $88 \ldots \ldots \ldots \ldots \ldots \ldots+\cdots$ & 1479 & 221315.8 & $-22 \quad 1744.4$ & 0.477 & $\leq 1.491$ & $\leq 12.869$ & 2.67 \\
\hline …........................... & 1479 & 221330.2 & -220333.8 & 0.677 & $\leq 1.531$ & $\leq 30.791$ & 4.30 \\
\hline 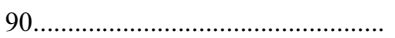 & 1694 & 221746.8 & +002244.4 & 0.602 & $\leq 0.905$ & $\leq 13.686$ & 4.56 \\
\hline 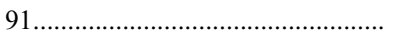 & 1694 & 221750.6 & +002132.4 & 0.227 & $\leq 0.873$ & $\leq 1.342$ & 4.17 \\
\hline 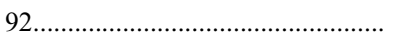 & 1694 & 221833.1 & +001737.2 & $0.332^{\mathrm{d}}$ & $\leq 0.743$ & $\leq 2.736$ & 15.48 \\
\hline 93 & 431 & 223955.2 & +033826.8 & 0.127 & $\leq 1.996$ & $\leq 0.850$ & 20.65 \\
\hline 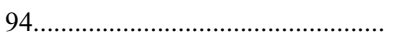 & 431 & 223956.5 & +033133.7 & 0.302 & $\leq 1.577$ & $\leq 4.651$ & 9.54 \\
\hline 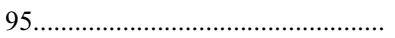 & 431 & 224025.0 & +033115.6 & 0.427 & $\leq 1.662$ & $\leq 11.022$ & 5.72 \\
\hline 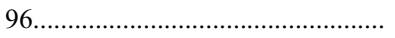 & 431 & 224025.4 & +033356.2 & 0.252 & $\leq 1.755$ & $\leq 3.420$ & 9.17 \\
\hline 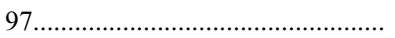 & 918 & 230251.8 & +085020.5 & 0.402 & $\leq 0.672$ & $\leq 3.868$ & 1.63 \\
\hline 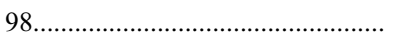 & 918 & 230309.4 & +084919.1 & 0.502 & $\leq 0.708$ & $\leq 6.906$ & 2.70 \\
\hline 99 & 861 & 234759.3 & +010344.5 & $0.248^{\mathrm{e}}$ & $\leq 0.822$ & $\leq 1.549$ & 2.83 \\
\hline 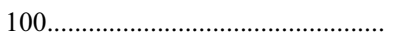 & 861 & 234815.8 & +005354.1 & $0.410^{\mathrm{e}}$ & $\leq 0.899$ & $\leq 5.423$ & 1.39 \\
\hline 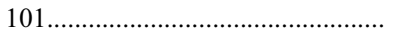 & 861 & 234839.6 & +010840.9 & 0.527 & $\leq 1.010$ & $\leq 11.075$ & 7.52 \\
\hline 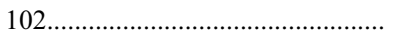 & 861 & 234935.6 & +005940.6 & 0.352 & $\leq 1.125$ & $\leq 4.736$ & 21.68 \\
\hline
\end{tabular}

NotE.-Units of right ascension are hours, minutes, and seconds, and units of declination are degrees, arcminutes, and arcseconds.

a Corrected redshifts estimated from red-sequence VTP unless otherwise noted.

b X-ray fluxes and luminosities are $3 \sigma$ upper limits for cluster candidates not detected in X-rays.

c Chandra PSF size at the location of optically detected source. The PSF is derived from the best-fit analytic relation between the Gaussian sigma of point sources and is similar to the radius encircling $50 \%$ of total counts for a monochromatic source at $0.75 \mathrm{keV}$.

${ }^{d}$ Redshift measurement from NED.

e Redshift measurement from ChaMP spectroscopic program.

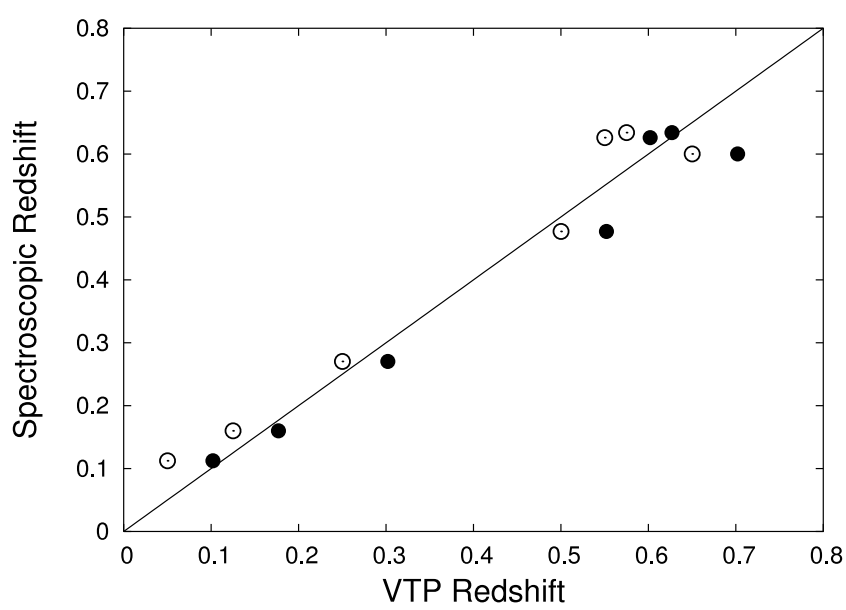

FIG. 6.-VTP redshifts with correction applied ( filled circles) and without correction (open circles). The application of the correction lowers the dispersion of the VTP-estimated redshifts from the corresponding spectroscopic measurements (solid line) from 0.05 to 0.03 .

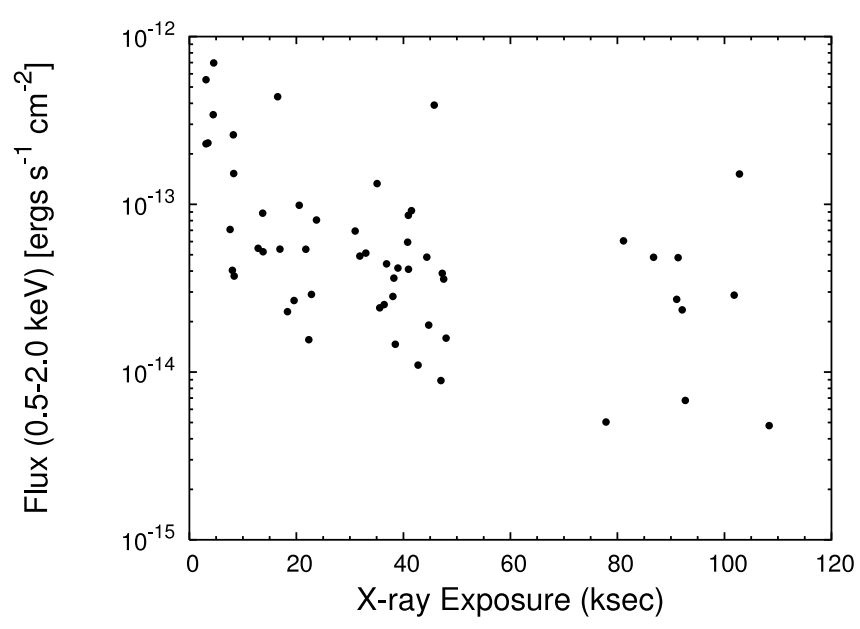

FIG. 7.-X-ray flux $(0.5-2 \mathrm{keV})$ of extended X-ray sources as a function of vignetting-corrected exposure time. Approximately $82 \%$ of the extended sources are detected from fields with exposure times $<50 \mathrm{ks}$. 


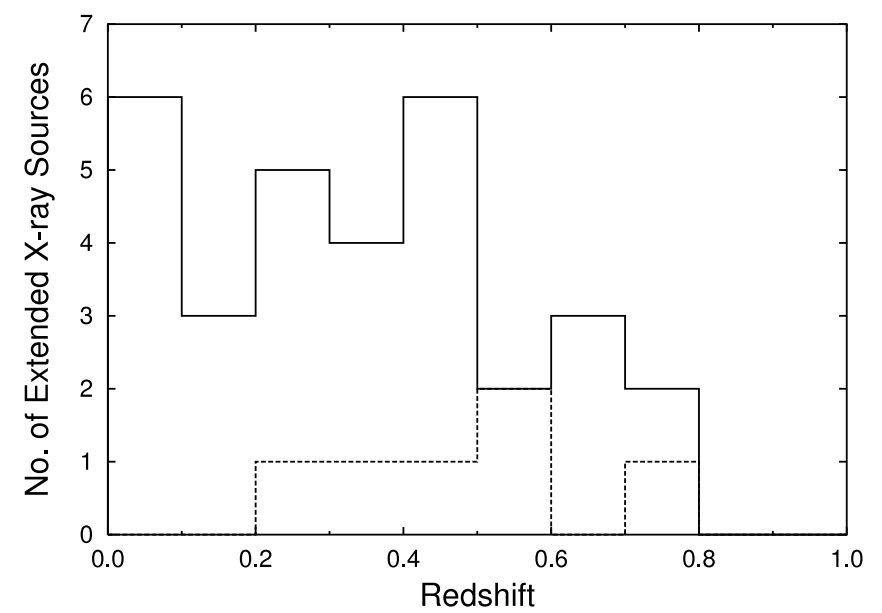

FIG. 8.-Redshift distribution of 31 extended X-ray sources with either spectroscopically (solid line) or VTP-estimated redshifts (dashed line). Six sources are associated with low-redshift single galaxies $(z<0.1)$. The redshift distribution for the cluster-only sample peaks at $z \sim 0.4$

the majority of our sources. A fit to the surface brightness profile with $\beta$ fixed at 0.67 yields a core radius accurate to $\pm 20 \%$ for $0.6<\beta<0.8$ (Jones \& Forman 1999). The X-ray surface brightness radial profile for a typical sample of nine extended sources is given in Figure 11. The best-fit $\beta$-model (solid line) along with the associated $1 \sigma$ uncertainty (dashed lines) are presented. In Figure 12 a histogram of the core radius distribution is presented for our 55 extended sources. The median angular core radius $\left(r_{c}=\right.$ $21.37 \pm 2$.'22) is depicted by the dashed line. If the six low $z$ galaxies are excluded, the median core radius increases to $r_{c}=$ $22.08 \pm 1$ ". 67 . This value is comparable to the typical core radius for extended sources detected using the ROSAT High Resolution Imager (HRI; Moretti et al. 2004) over a similar flux and redshift range.

To search for possible evolution in the measured core radius as a function of redshift, we plot in Figure 13 the angular core radius (arcseconds) and the metric core radius (kiloparsecs) as a function of redshift. The top panel reveals that the detectability of clusters of different observed core radii is not a strong function of redshift. When we plot the core radii in linear units (bottom), no direct correlation with redshift is observed for our sample. This result is in agreement with the study of Vikhlinin et al. (1998a), which found no obvious evolution of the core radius with redshift for a sample of 203 clusters from the 160 Square Degree ROSAT Survey. However, we do see an increase in the dispersion of core radii with redshift in the bottom panel of Figure 13. This may be a volume effect - more luminous clusters with larger core radii are rare and therefore only well-sampled at higher redshifts.

The core radius versus X-ray luminosity is plotted in Figure 14 for the 31 extended X-ray sources with either spectroscopic or VTP estimated redshifts. A correlation exists between the core radius and $L_{\mathrm{X}}$ in the sense that the more $\mathrm{X}$-ray luminous sources are physically more extended. A correlation between the core radius and X-ray luminosity is not unexpected given that the physical size of X-ray clusters has been found to increase with X-ray temperature and hence luminosity (Mohr et al. 2000). This effect was also seen by Jones \& Forman (1999) for a heterogeneous sample of 368 low-redshift $(z<0.2)$ X-ray clusters imaged by Einstein. To determine which of the variables - redshift, $L_{\mathrm{X}}$, or $f_{\mathrm{X}}$ - presents the primary correlation with core radius $r_{c}$, we apply partial correlation analysis to the 31 serendipitous $\mathrm{X}$-ray-extended source detections with redshift measurements (from either spectroscopy or VTP redshift estimates). For our sample of $N=31$, the correlation of $r_{c}$ with $L_{\mathrm{X}}$ remains highly significant when holding either $f_{\mathrm{X}}$ or redshift constant. While, as expected, our effective flux limit creates the strongest simple correlation between $L_{\mathrm{X}}$ and redshift, of all the other combinations tested, $r_{c}$ depends most strongly on $L_{\mathrm{X}}$ (partial Kendalls $\tau=$ 0.439 with $\sigma=0.116$; Akritas \& Siebert 1996). A power-law fit to the X-ray luminosity-redshift data yields $r_{c} \propto L_{\mathrm{X}}^{0.48 \pm 0.04}$ and is displayed as the solid line in Figure 14.

Galaxy mergers are expected to have a greater impact on galaxy evolution in the group environment rather than at the center of rich clusters due to the lower velocity dispersion of the group members (e.g., Dubinski 1998). Simulations predict that the end result of galaxy mergers in groups will probably be the formation of a single elliptical galaxy with an extended X-ray halo (Mamon 1987; Barnes 1989). These "fossil groups" provide important information on the evolution of galaxies and the ICM in these type of locales (Ponman et al. 1994; Vikhlinin et al. 1999; Jones et al. 2000; Ulmer et al. 2005). An expected signature of fossil groups is the presence of extended X-ray emission centered on a luminous early-type galaxy. To determine whether our six single galaxies with extended X-ray emission are fossil groups, we compared the spatial extent of the X-ray and optical emission. For all six galaxies the X-ray emission is more concentrated than the optical light, with no evidence to suggest that some fraction of the X-ray emission is due to a group ICM. We thus conclude that none of our six galaxies are associated with a fossil group.

\subsection{Comparison of X-Ray and Optical Clusters}

We cross-correlated the extended X-ray source catalog, compiled from the wavelet decomposition technique, with the optical cluster sample constructed from the red-sequence-filtered VTP method. From the sample of 55 extended X-ray sources, 6 were found to be associated with low-redshift galaxies. Of the 49 X-ray extended sources not associated with low-redshift galaxies, only 28 are located in X-ray fields that overlap with our MOSAIC pointings (6.1 $\mathrm{deg}^{2}$; see Table 2$)$. The fraction of matches between the X-ray and optical cluster catalogs is $46 \%$ (13 out of 28 ), with a median redshift of $z_{\text {med }}=0.477 \pm 0.202$, where the uncertainty is the rms of the dispersion. In Table 5 we tabulate source information from the literature for objects near the position of each extended X-ray source as archived by the NASA/IPAC Extragalactic Database (NED). ${ }^{12}$

A $46 \%$ match of the X-ray clusters to our optical VTP sources may not seem surprising, given that the variation in the X-ray and optical exposure times (see Tables 1 and 2) affects the limiting flux reached in either passband. To test whether optical magnitude limits have a direct impact on the matched rate for our sample, the turnover magnitude ${ }^{13}$ for fields containing matched clusters is compared to fields without matched sources (see Table 2 for the turnover magnitudes for each field). The turnover magnitude for the fields with optical/X-ray matches varies from $i^{\prime}=23.12$ to $24.62 \mathrm{mag}$, with a median value of $23.62 \pm 0.41$. For the optical fields containing no detected extended X-ray sources, the turnover magnitude varies from $i^{\prime}=22.12$ to $24.62 \mathrm{mag}$, with a median value of $23.62 \pm 0.78$. The equality of the median turnover magnitudes for the optical fields containing detected and nondetected X-ray sources (most of the fields are identical between

12 This research has made use of NED, which is operated by the Jet Propulsion Laboratory, California Institute of Technology, under contract with the National Aeronautics and Space Administration.

13 The turnover magnitude is the magnitude at which the differential galaxy counts begin to decrease with increasing magnitude due to incompleteness. 
TABLE 5

Published Sources near X-Ray Positions ${ }^{a}$

\begin{tabular}{|c|c|c|}
\hline $\begin{array}{l}\text { Source Name } \\
\text { CXOMP }\end{array}$ & Published $\mathrm{ID}^{\mathrm{b}}$ & Comments $^{\mathrm{c}}$ \\
\hline J002650.2+171935 & Zw Cl 0024.0+1652:[CKS2001] 541 & Background group $\left(6^{\prime \prime}\right)^{\mathrm{d}}$ \\
\hline 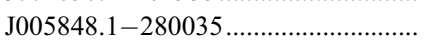 & 2MASX J00584850-2800414 & Single galaxy: BCG? $\left(6^{\prime \prime}\right)$ \\
\hline 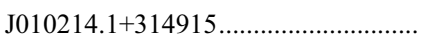 & 2MASX J01021352+3149243 & Single galaxy: BCG? $\left(12^{\prime \prime}\right)$ \\
\hline 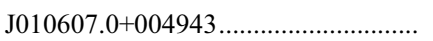 & SDSS CE J016.528793+00.817471 & Possible association $\left(42^{\prime \prime}\right)$ \\
\hline 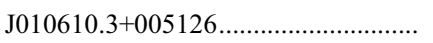 & SDSS J010610.38+005120.4 & Galaxy $\left(6^{\prime \prime}\right)$ \\
\hline 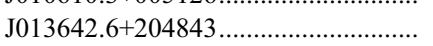 & [B2002a] 02 & DSS: faint galaxies near X-ray position $\left(12^{\prime \prime}\right)$ \\
\hline 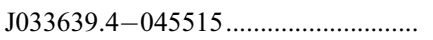 & & \\
\hline 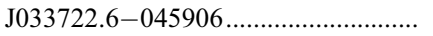 & 2MASX J03372263-0459055 & Single spiral galaxy $\left(0^{\prime \prime}\right)$ \\
\hline 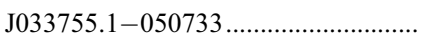 & Abell 447 & Galaxy cluster $\left(60^{\prime \prime}\right)$ \\
\hline 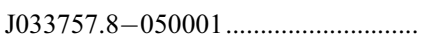 & 2MASX J03375780-0500006 & Galaxy $\left(0^{\prime \prime}\right)$ \\
\hline 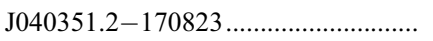 & APMUKS(BJ) B040135.74-171628.1 & Galaxy $\left(6^{\prime \prime}\right)$ \\
\hline 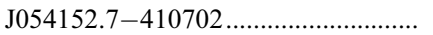 & & $\ldots$ \\
\hline 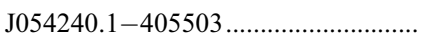 & RX J0542.8-4100 & Galaxy cluster $\left(6^{\prime \prime}\right)$ \\
\hline 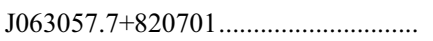 & 1WGA J0630.7+8206 & $\mathrm{X}$-ray source $\left(30^{\prime \prime}\right)$ \\
\hline 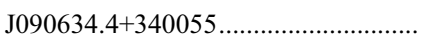 & RIXOS F257_037 & $\operatorname{AGN}\left(12^{\prime \prime}\right)$ \\
\hline 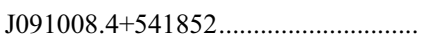 & CXOU J0910.1+5419: [B2002a] 14 & Galaxy cluster $\left(0^{\prime \prime}\right)$ \\
\hline 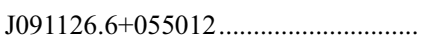 & RX J0911.4+0551 & Galaxy cluster $\left(0^{\prime \prime}\right)$ \\
\hline 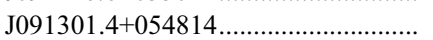 & & \\
\hline 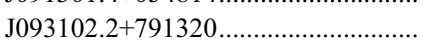 & [B2002a] 15 & Extended X-ray source $\left(6^{\prime \prime}\right)$ \\
\hline 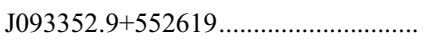 & 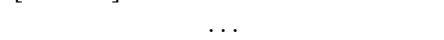 & $\ldots$ \\
\hline 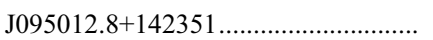 & $\ldots$ & $\ldots$ \\
\hline 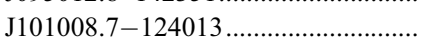 & LCRS B100740.9-122545 & Galaxy $\left(24^{\prime \prime}\right)$ \\
\hline 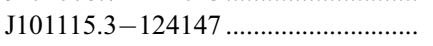 & $\ldots$ & - \\
\hline $\mathrm{J} 105624.6-033517 \ldots \ldots \ldots \ldots \ldots \ldots \ldots \ldots$ & XBS J105624.2-033522 & Galaxy $\left(6^{\prime \prime}\right)$ \\
\hline 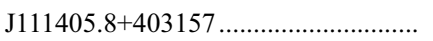 & & \\
\hline 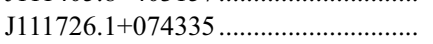 & RX J1117.4+0743 & Galaxy cluster $\left(0^{\prime \prime}\right)$ \\
\hline 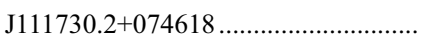 & RIXOS F258_101 & Galaxy cluster $\left(30^{\prime \prime}\right)$ \\
\hline 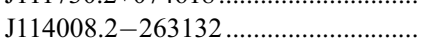 & {$[\mathrm{B} 2002 \mathrm{a}] 22^{-}$} & Extended X-ray source: galaxy cluster $\left(0^{\prime \prime}\right)$ \\
\hline 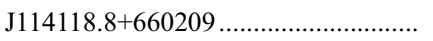 & & $\ldots$ \\
\hline 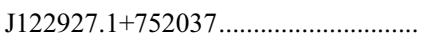 & 1WGA J1229.6+7520 & $X$-ray source $\left(36^{\prime \prime}\right)$ \\
\hline 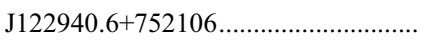 & 1WGA J1229.6+7520 & $\mathrm{X}$-ray source $\left(30^{\prime \prime}\right)$ \\
\hline 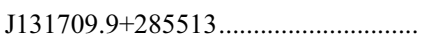 & CXOSEXSI J131710.0+285516 & $\left(6^{\prime \prime}\right)$ \\
\hline 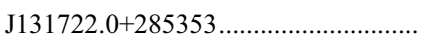 & 2MASX J13172206+2853460 & Elliptical galaxy $\left(6^{\prime \prime}\right)$ \\
\hline 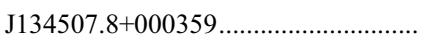 & 2QZ J134507.4+000406 & Galaxy $\left(12^{\prime \prime}\right)$ \\
\hline 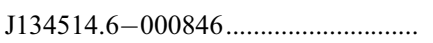 & [DDM2004] J134515.60-000830.8 & Galaxy cluster $\left(24^{\prime \prime}\right)$ \\
\hline 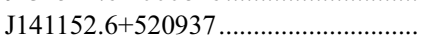 & CXOSEXSI J141153.0+521020 & $X$-ray source $\left(42^{\prime \prime}\right)$ \\
\hline 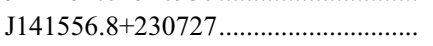 & OC03 J1415+2307 & Galaxy cluster $\left(12^{\prime \prime}\right)$ \\
\hline $\mathrm{J} 141602.1+230647 \ldots \ldots \ldots \ldots \ldots \ldots \ldots \ldots \ldots$ & 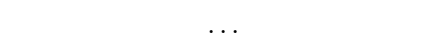 & 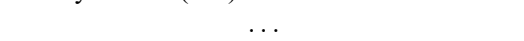 \\
\hline $\mathrm{J} 153259.2-004414 \ldots \ldots \ldots \ldots \ldots \ldots \ldots \ldots \ldots$ & $\ldots$ & $\ldots$ \\
\hline $\mathrm{J} 153415.0+232459 \ldots \ldots \ldots \ldots \ldots \ldots \ldots \ldots \ldots$ & $\ldots$ & $\ldots$ \\
\hline 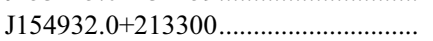 & [B2002a] 30 & X-ray source: galaxy cluster $\left(36^{\prime \prime}\right)$ \\
\hline 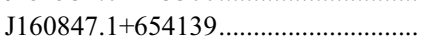 & 2MASX J16084763+6541402 & Galaxy $\left(6^{\prime \prime}\right)$ \\
\hline 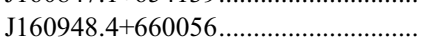 & 1WGA J1609.7+6600 & $X$-ray source $\left(30^{\prime \prime}\right)$ \\
\hline $\mathrm{J} 165514.4-082944 \ldots \ldots \ldots \ldots \ldots \ldots \ldots \ldots \ldots$ & $\ldots$ & (1) \\
\hline 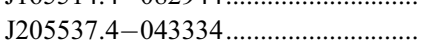 & CXOSEXSI J205537.3-043333 & $\mathrm{X}$-ray source $\left(0^{\prime \prime}\right)$ \\
\hline 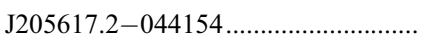 & CXOSEXSI J205617.1-044155 & X-ray source $\left(0^{\prime \prime}\right)$ \\
\hline 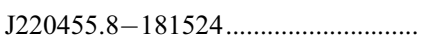 & $\ldots$ & \\
\hline 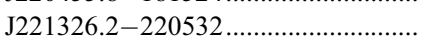 & IC 1435 & S-galaxy: X-ray coincident with S-arm $\left(18^{\prime \prime}\right)$ \\
\hline 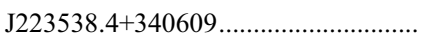 & 1WGA J2235.6+3406 & $X$-ray source $\left(12^{\prime \prime}\right)$ \\
\hline 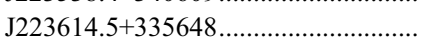 & CXOU J223615.0+335630 & $\mathrm{X}$-ray source $\left(18^{\prime \prime}\right)$ \\
\hline 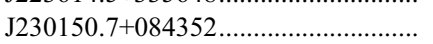 & S & \\
\hline 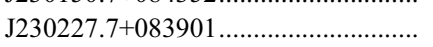 & & \\
\hline 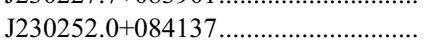 & 2MASX J23025207+0841356 & Single E-galaxy $\left(0^{\prime \prime}\right)$ \\
\hline 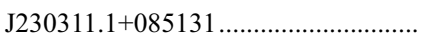 & & \\
\hline J234817.8+010617 & 2MASX J23481801+0106174 & Single E-galaxy $\left(0^{\prime \prime}\right)$ \\
\hline
\end{tabular}

${ }^{\text {a }}$ Published references acquired from NED.

b Blank field if published sources $>2$ ' from object position.

${ }^{c}$ Available redshifts tabulated in Table 3.

d Distance in arcseconds from extended X-ray centroid is shown in parentheses. 


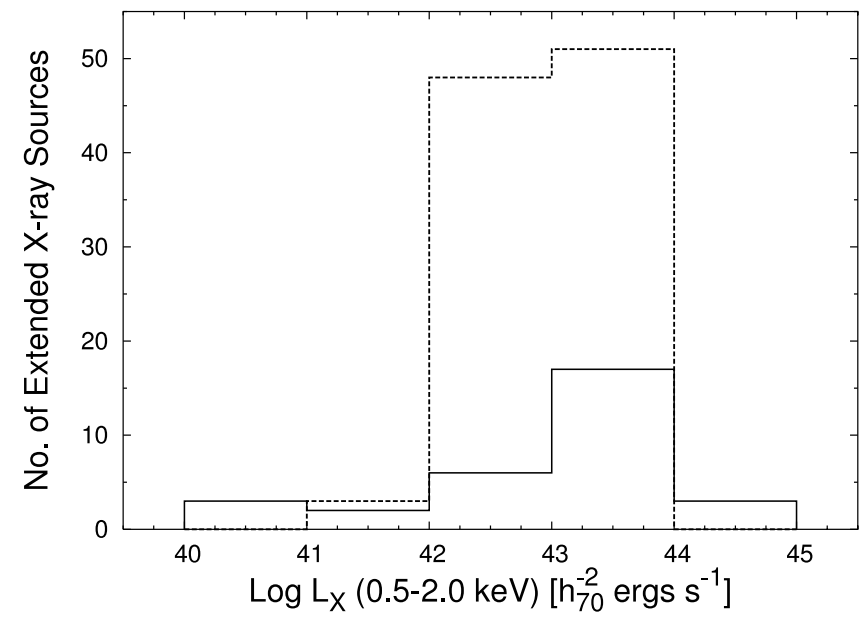

FIG. 9.-Distribution of X-ray luminosity for 31 serendipitously detected extended X-ray sources (solid line) and distribution of upper limits to the X-ray luminosity for 102 cluster candidates detected by VTP but not in the X-rays (dashed line).

the two samples) suggests that we are not missing a large fraction of our extended X-ray sources in the optical due to a variation in the optical magnitude limit between the two samples.

A possible explanation for the $46 \%$ match rate between our $\mathrm{X}$-ray clusters and optical VTP counterparts is that the X-ray flux limit is much fainter relative to the optical limit for fields that lack optical matches than for fields containing matched sources. However, a comparison of our measured flux limit ratios indicates that the difference between the matched and unmatched samples are statistically insignificant. Indeed, the ChaMP optical follow-up was originally designed to probe similar $f_{\mathrm{X}} / f_{o}$ populations in each field by tuning optical magnitude limits to the $\mathrm{X}$-ray exposure times for each field (Green et al. 2004).

In Figure 15 we plot the $i^{\prime}$-band magnitude optical field limit versus X-ray flux for the 28 extended X-ray sources (excluding the low- $z$ galaxies) that overlap our optical fields. From this figure we see that for optical fields with turnover magnitude limits brighter than $i^{\prime}=23$, all four extended X-ray sources are not detected in the optical data by VTP (optical data is too shallow to allow a robust detection). The area of the plot having X-ray sources with bright X-ray fluxes and faint optical field limits contains several sources that are undetected by VTP. If we restrict ourselves to fields with optical magnitude limits fainter than $i^{\prime}=$ 23 and $f_{\mathrm{X}}>10^{-14} \mathrm{ergs} \mathrm{s}^{-1} \mathrm{~cm}^{-2}$, we find $10 \mathrm{X}$-ray sources without optical matches. A detailed visual inspection of our optical images coincident with these 10 sources shows that 2 are most likely undetected by VTP due to the presence of a nearby bright star, 4 sources have very faint galaxies near the X-ray centroid but too faint to be flagged by VTP, and the remaining 4 show no conclusive evidence of being clusters in the optical (i.e., the area looks "fieldlike" in nature). These undetected extended X-ray sources are prime candidates for deeper optical/near-IR followup imaging.

Our VTP red-sequence detection may be missing optical clusters with a large fraction of blue galaxies. In addition, there is the possibility that some of the unmatched X-ray sources are high- $z$ clusters whose galaxies are too faint in these optical filters to be detected by our VTP algorithm. Of the 15 extended X-ray sources without VTP optical matches, only 3 have measured spectroscopic redshifts $(z=0.3768,0.4118$, and 0.4256$)$, with X-ray luminosities ranging from $(4.4-26) \times 10^{42} \mathrm{ergs} \mathrm{s}^{-1}$. These three extended X-ray sources have X-ray point sources embedded

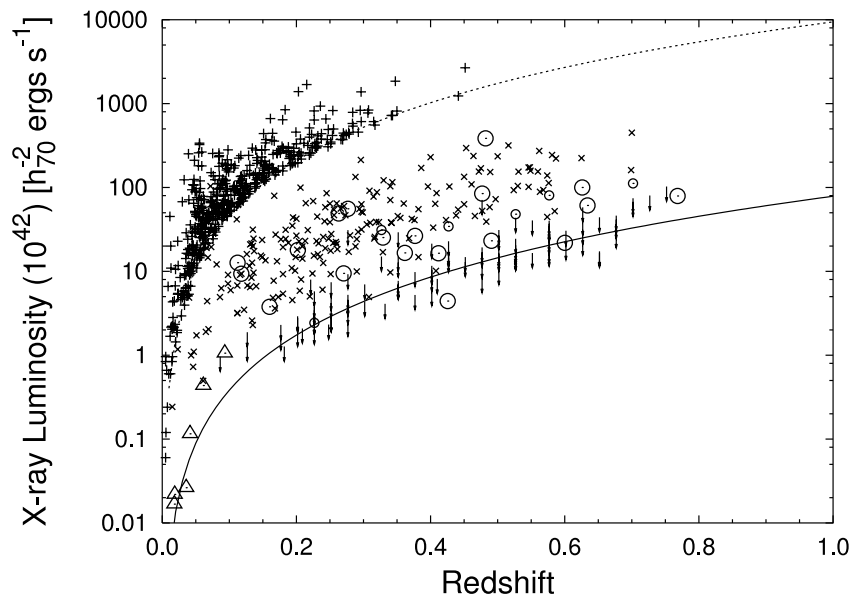

FIG. 10.-Distribution of X-ray luminosity $(0.5-2 \mathrm{keV})$ as a function of redshift. The open circles mark the 25 extended X-ray sources associated with clusters with redshifts, while the open triangles depict the 6 low- $z$ galaxies. The arrows represent X-ray luminosity upper limits for the 102 sources detected by VTP but undetected in the X-rays. The plus signs depict the $447 \mathrm{X}$-rays clusters from the REFLEX compilation, while 200 clusters from the 160 Square Degree ROSAT Survey are represented by times crosses. The solid line indicates the flux limit of $1.5 \times 10^{-14} \mathrm{ergs} \mathrm{s}^{-1} \mathrm{~cm}^{-2}(0.5-2 \mathrm{keV})$, which is shown for illustrative purposes. The flux limit of the REFLEX sample (converted to the $0.5-2 \mathrm{keV}$ energy band) is shown as the dashed line, corresponding to $1.8 \times 10^{-12} \mathrm{ergs} \mathrm{s}^{-1}$ $\mathrm{cm}^{-2}$. Larger symbols represent sources with spectroscopic redshifts, while sources with VTP-only-estimated redshifts are marked with smaller symbols.

within them and were targets of the ChaMP spectroscopic AGN follow-up program. These sources were not detected by VTP, since only a couple galaxies were observed within the redsequence slice consistent with the redshift of the extended X-ray source ( 2 galaxies for the $z=0.3768$ source, 3 galaxies for the $z=0.4118$ source, and 1 galaxy for the $z=0.4256$ source). To test whether the remaining 12 sources have properties consistent with our X-ray/optically matched sample, we include these objects on a plot of $L_{X}$ versus redshift (see Fig. 16), which is similar to Figure 10. To estimate a redshift, we simply use the extinctioncorrected $i^{\prime}$ magnitude of the galaxy located closest to the X-ray centroid and assume that it is an $M^{*}$ elliptical galaxy. We apply $K$ - and evolution corrections based on a Bruzual \& Charlot (2003) passive evolution model for early-type galaxies with a formation redshift of $z=3$. Figure 16 shows that 5 of the 12 sources would have properties consistent with $z>0.8$ clusters and thus could be at redshifts greater than our X-ray/optically matched sample. If we assume the galaxies have $L>L^{*}$, as might be expected for the brightest cluster galaxy, this would increase the estimated redshift for each source (changing $z_{f}=3$ to 5 will have the opposite effect). The depth of the optical imaging for these clusters may be inadequate to allow us to use VTP to detect these high- $z$ sources because fainter galaxies are beyond the optical limit. These objects are prime high-redshift cluster candidates, which we are pursuing with deep imaging in near-infrared bands (e.g., FLAMINGOS $J$ - and $K_{s}$-band observations). At low- $z$, five of the seven sources are either near bright stars or are located on shallow optical images that are only complete to $i^{\prime} \sim 22$, which may explain their nondetection using VTP. For these we will seek deeper imaging.

Our optical VTP cluster catalog, generated from the identical $6.1 \mathrm{deg}^{2}$ sky area covered by our Chandra fields, contains 115 sources, with a median redshift of $z=0.427 \pm 0.013$. Only 13 of these optical clusters are detected in the X-rays as extended sources, and thus $89 \%$ (102 out of 115) of our VTP detections are not included in the X-ray cluster catalog (see Table 4). The median redshift of the optical clusters not detected in the X-rays is 


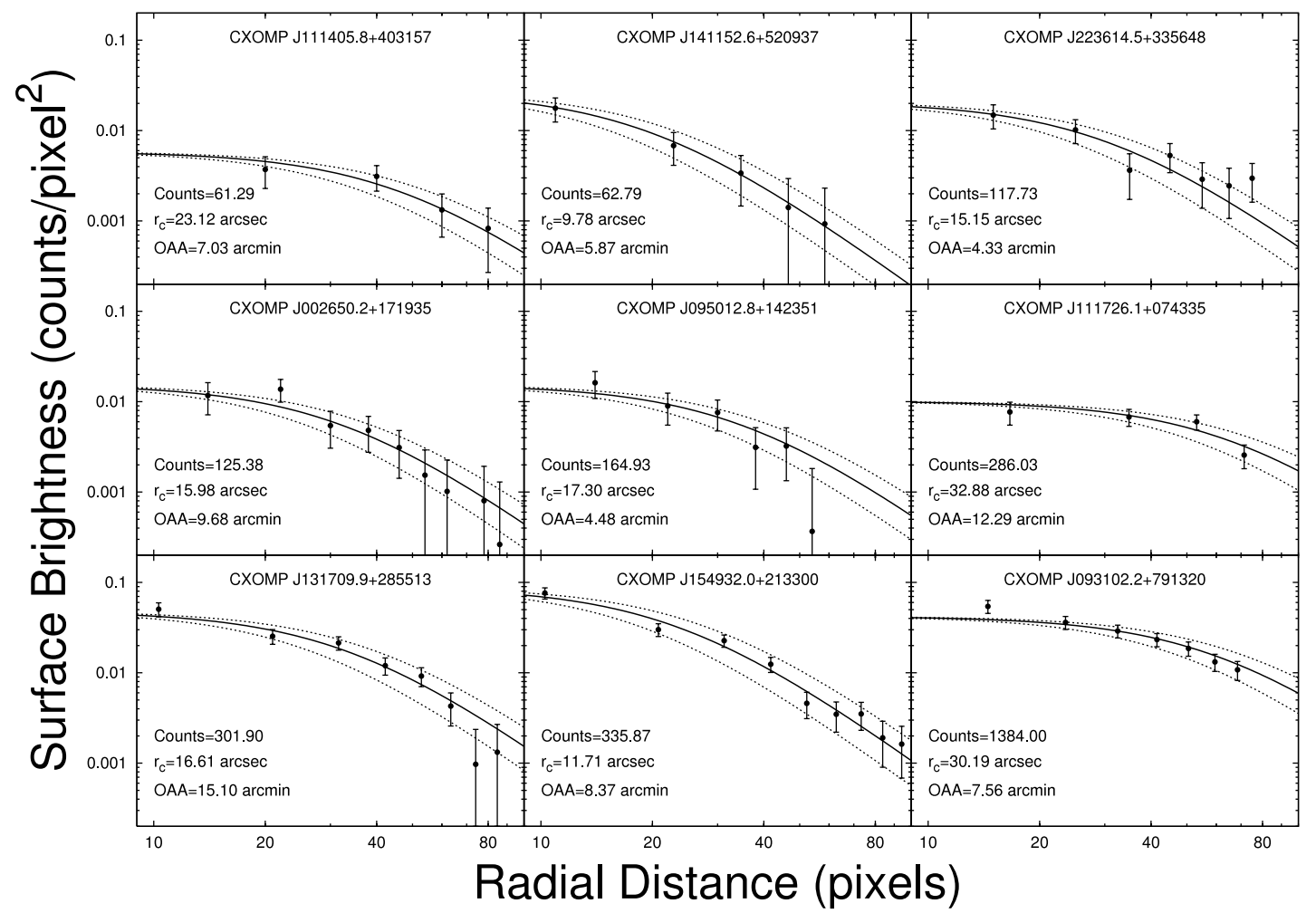

Fig. 11. - X-ray surface brightness radial profiles for a representative sample of nine extended sources. The best-fit $\beta$ model $(\beta=0.67$; solid line) along with the associated $1 \sigma$ uncertainty (dashed lines) are presented for each source. The depicted sources range in total counts from 61.29 to 1384 and core radii extending from 9.78 to 32".88. The total counts, core radius, and off-axis angles are tabulated in Table 4 for each extended X-ray source.

$z=0.427 \pm 0.010$. Redshift estimates are derived from the redsequence-filtered VTP method or measured from our ChaMP spectroscopic program. An example of a matched X-ray/optical source is presented in Figure 17 for CXOMP J105624.6033517. This extended object also includes an X-ray point source, a previously known quasar at $z=0.626$ (Table 5). Figure 17 displays the $i^{\prime}$-band optical image with the X-ray contours overlaid. The detection of extended emission in the presence of a bright point source highlights the advantage of Chandra's spatial resolution in serendipitous cluster samples.

In Figure 18 the histogram redshift distribution of the optical cluster detections - regardless of X-ray matches - is depicted.

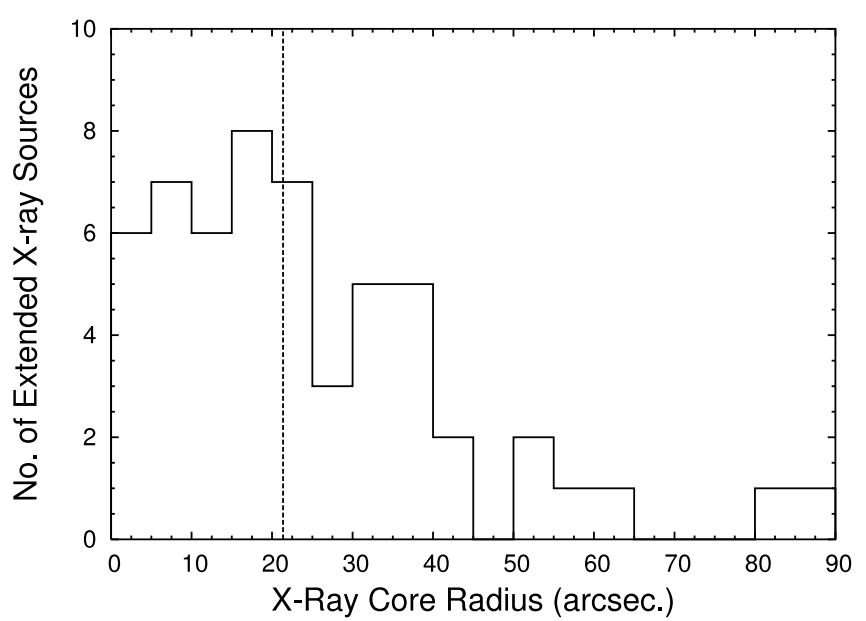

Fig. 12.-Histogram distribution of the measured core radius for 55 extended X-ray sources. The median core radius of 21 ".37 is represented by the vertical dashed line.

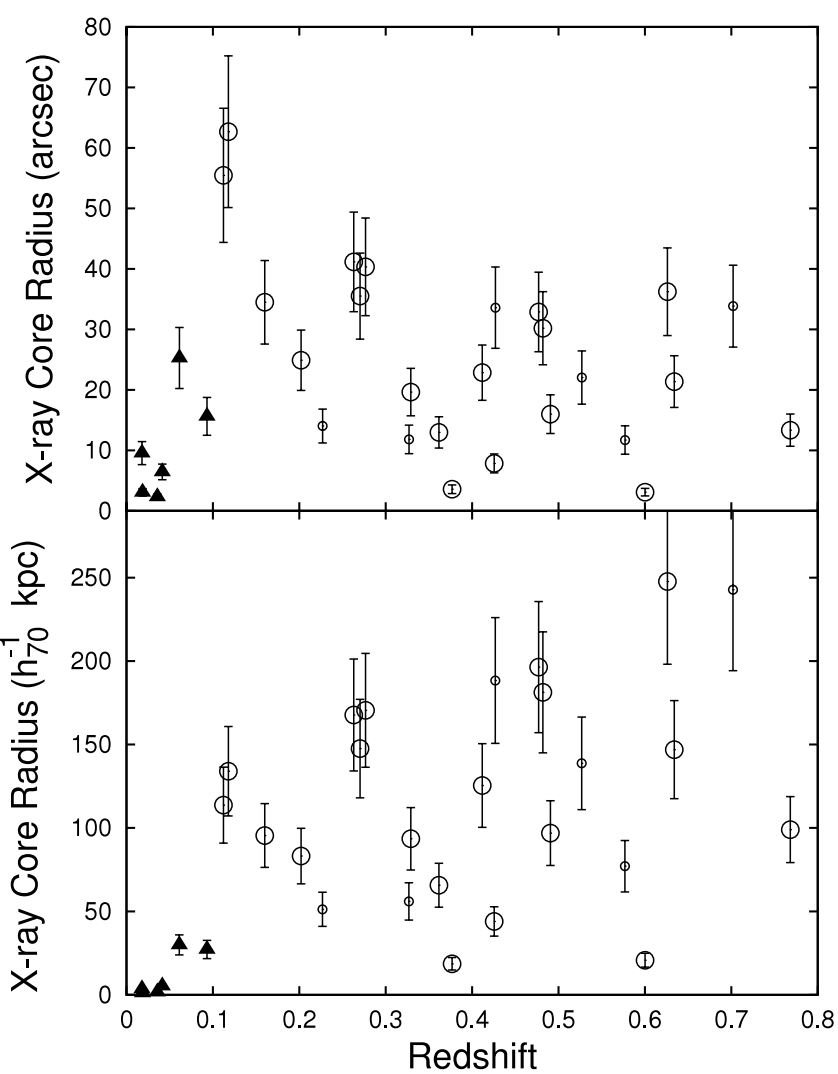

FIG. 13.-Angular (arcseconds) and physical core radius $\left(h_{70}^{-1} \mathrm{kpc}\right)$ for $31 \mathrm{ex}-$ tended X-ray sources as a function of redshift. The filled triangles depict extended $\mathrm{X}$-ray sources associated with single galaxies, and open circles represent X-raydetected clusters; large symbol size indicate sources with spectroscopic redshifts, while the smaller symbols represent objects with red-sequence-filtered VTP redshifts. No obvious correlation is apparent between the core radius and redshift. 


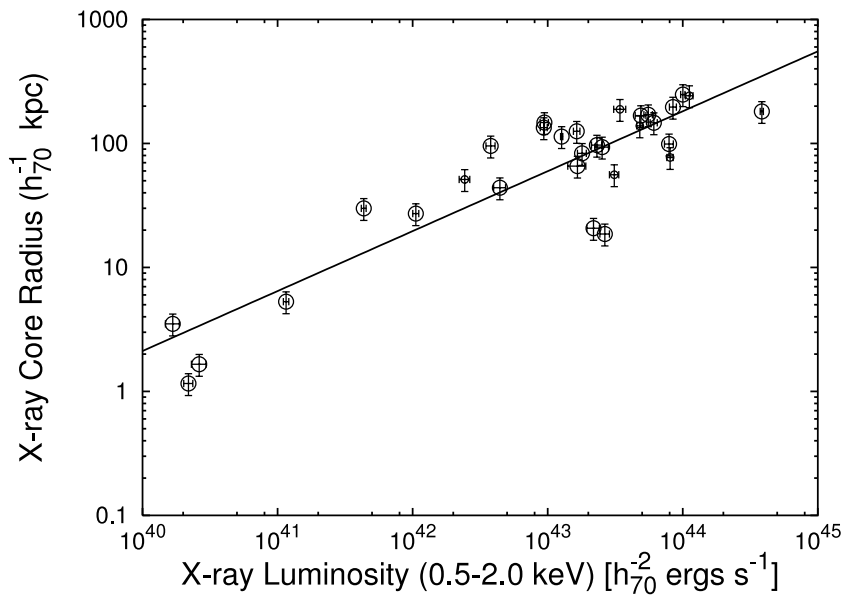

FIg. 14.- Metric core radius $\left(h_{70}^{-1} \mathrm{kpc}\right)$ as a function of X-ray luminosity for the 31 extended X-ray sources with estimated redshifts. The core radius and luminosity are well correlated with a best-fit power law (solid line) of $r_{c} \propto$ $L_{\mathrm{X}}^{0.48 \pm 0.04}$. Sources with spectroscopic redshifts are depicted with the large symbols, and objects with VTP estimated redshifts are shown with the smaller symbols.

The redshift distribution of these optical clusters is very similar to the distribution shown in Figure 8 for the X-ray extended sources (i.e., most of the clusters range in redshift from $0.2<$ $z<0.8$ ). This is expected given that the red-sequence-filtered VTP technique provides reliable redshifts to $z \sim 0.7$ (see $\S 3.2 .1$ ).

For the optical cluster candidates without an X-ray match, we calculated the $\mathrm{X}$-ray upper limits $(0.5-2 \mathrm{keV})$, as described previously in $\S 4.2$. Table 4 lists the coordinates, redshifts (VTP or spectroscopic), X-ray flux upper limits, X-ray luminosity upper limits, and Chandra PSF sizes for all 102 cluster candidates. The upper limits are $3 \sigma$ values derived from background-subtracted counts measured within a $84^{\prime \prime}$ radius aperture (extrapolated to infinity using the $\beta$ model), with point sources masked out and Poisson statistics assumed (see Figs. 9 and 10 for a comparison between the upper limits of the X-ray luminosity and values measured for X-ray-detected extended sources).

The difference between the number of X-ray/optical matches (13 sources) versus optical-only detections (102 candidates) can-

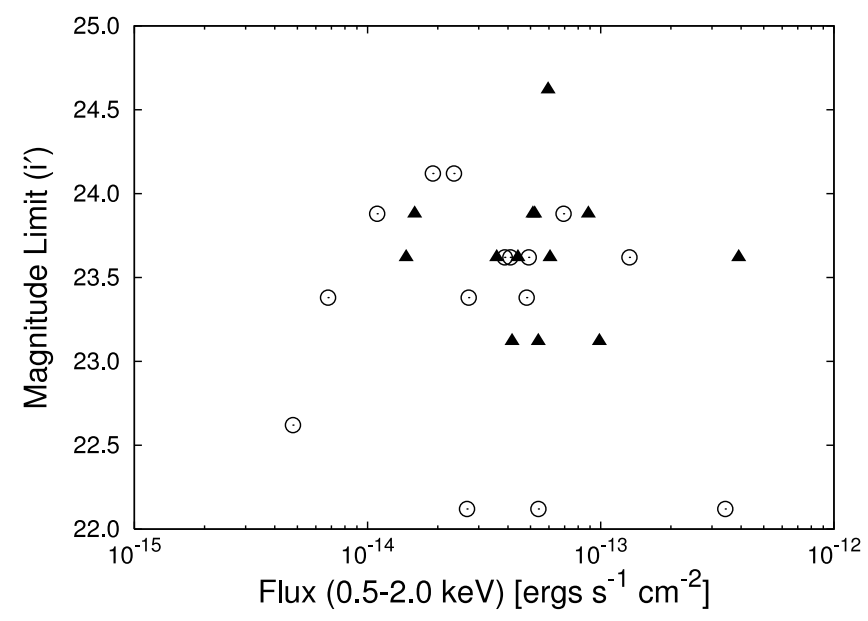

Fig. 15.-The $i^{\prime}$-band optical field turnover magnitude vs. X-ray flux for the 28 extended X-ray sources that overlap with the mosaic imaging (the 6 low- $z$ galaxies are excluded). The 13 matched optical/X-ray sources are depicted as the filled triangles, while the $15 \mathrm{X}$-ray sources without optical VTP matches are represented by the open circles. Several non-VTP detected sources with faint optical magnitude field limits and bright $f_{\mathrm{X}}$ are prime candidates for deeper optical/near-IR follow-up imaging to detect high-redshift clusters.

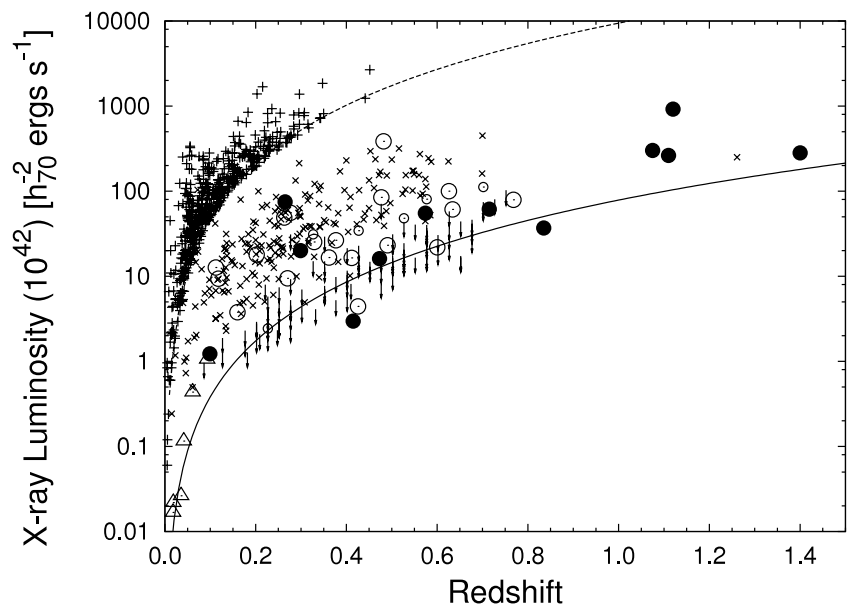

FIG. 16.-Distribution of X-ray luminosity $(0.5-2 \mathrm{keV})$ as a function of redshift. This plot is similar to Fig. 10 and includes 12 extended X-ray sources that have no VTP-detected optical counterparts or measured redshifts ( filled circles). Redshifts for these sources have been estimated using the $i^{\prime}$-band magnitude of the galaxy nearest to the X-ray centroid.

not be explained as simply the difference in the spurious detection rates expected for both detection methods $(<10 \%$ for the X-ray wavelet decomposition technique vs. $<20 \%$ for the red-sequencefiltered VTP method). A possible explanation is that the optical detection method is more sensitive to poor clusters and groups, which would be expected to contain little hot gas and thus be weak X-ray emitters. The dashed line in Figure 9 represents the upper limit of the X-ray luminosity for those optical detections not matched to the extended X-ray source catalog. The distribution of the X-ray luminosity upper limits is consistent with a population of groups and "normal" clusters (Mulchaey 2000).

A possible contributing factor for the disparity in the number of X-ray- and optical-only detections is that the X-ray counterparts to the optical sources have pointlike X-ray emission and thus are not flagged as extended sources. This scenario can be checked by cross-correlating the X-ray point source positions

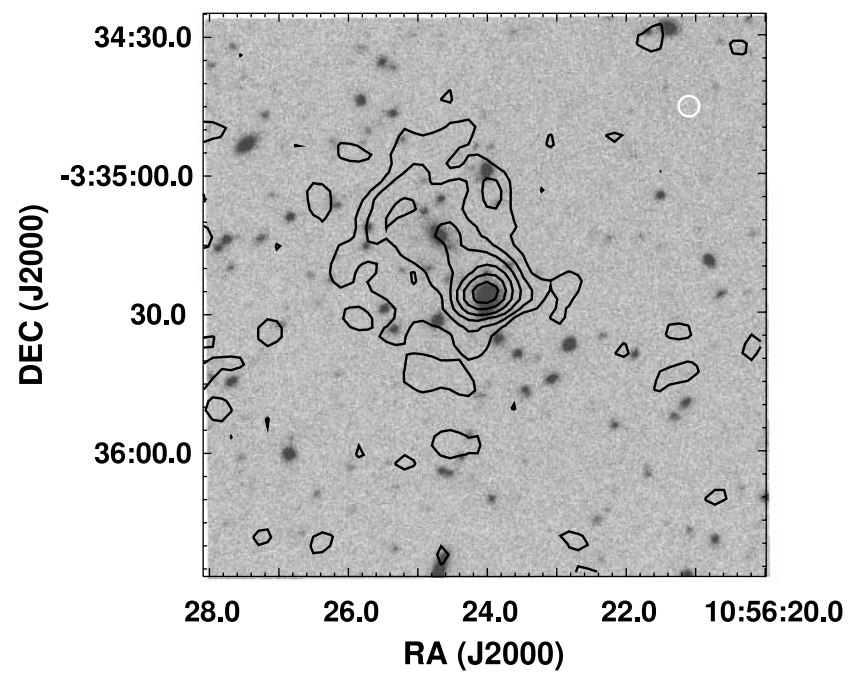

FIG. 17.-Optical $i^{\prime}$-band image of extended X-ray source CXOMP $\mathrm{J} 105624.6-033517$ (see Table 1) with the X-ray contours overlaid. This is an example of an extended X-ray source with an X-ray point source (a known $z=$ 0.626 quasar) embedded within. The size of the Chandra PSF at the source offaxis angle $(8.8)$ is $\sim 4.5$ and is represented by the white circle in the top right corner of the figure. 


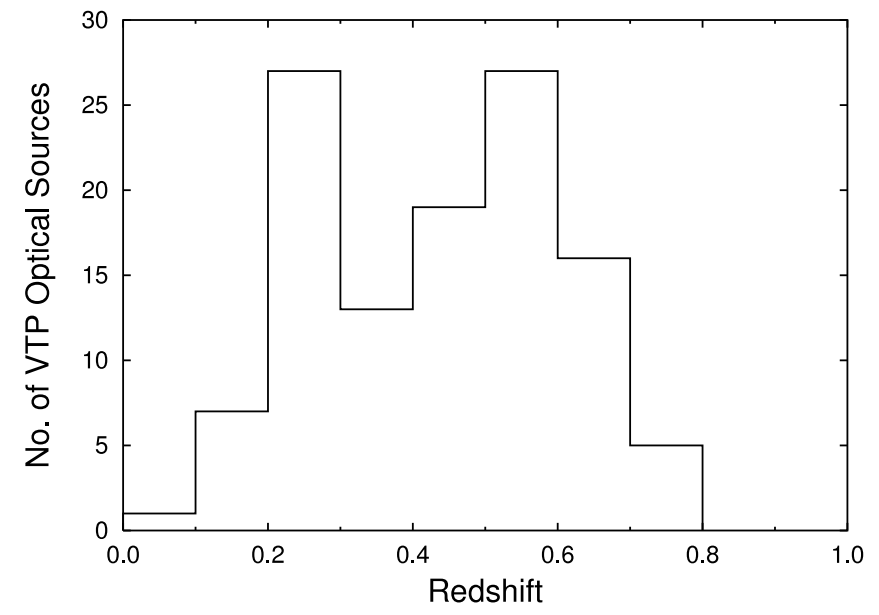

FIG. 18.- Redshift distribution of 115 red-sequence-filtered VTP optical detections of which 13 have X-ray counterparts. The VTP redshift estimates are based on the redshift of the associated red-sequence slice that maximizes the confidence and probability of being a real cluster. When available, spectroscopic redshifts are used in lieu of the VTP estimates.

from ChaMP (Kim et al. 2004a) with the optical cluster candidates not matched to the extended X-ray source compilation. Of the 102 optical clusters without extended X-ray matches, 9 are detected by ChaMP as X-ray point sources. We derive extended source flux upper limits for these, as described in $\S 4.2$, but we exclude the X-ray point source (conservatively using the $90 \%$ encircled counts radius). The resulting median X-ray luminosity of these 9 upper limits is $(3.87 \pm 12.74) \times 10^{42} \mathrm{ergs} \mathrm{s}^{-1}$. Thus, it is reasonable to assume that $\sim 9 \%$ (9 out of 102) of the opticalonly cluster candidates are not detected as extended X-ray sources because their X-ray emission is pointlike, thus excluding them from our extended X-ray source catalog. To check whether the Chandra PSF size has a significant impact on the fraction of X-ray-to-optical detections, we plot in Figure 19 the histogram of sources by Chandra PSF size both for the sample of optical clusters not detected as an extended X-ray source and for all 55 extended X-ray sources. Figure 19 gives the visual appearance that the two distributions are consistent. The two-sample Kolmogorov-Smirnov (K-S) test yields $D=0.14$, with a $55 \%$ probability that the null hypothesis cannot be rejected (i.e., confirming that the histograms are not inconsistent). A small number of truly extended sources may also be missed if they host bright embedded point sources. Both detailed simulations and a larger Chandra-selected cluster sample would help to constrain this contribution. ChaMP has initiated such a study, which is beyond the scope of the current paper.

Finally, there is the possibility that some fraction of the opticalonly detections are due to the chance alignment of filaments in the large-scale structure of the cosmic web of galaxies (Gladders \& Yee 2000). Since our optical cluster candidates are detected by filtering with respect to the color-magnitude red sequence, we minimize false optical detections. In Table 6 we tabulate information available from NED regarding sources located near each optical source not detected in the X-rays.

\subsubsection{Optical Cluster Richness}

Galaxy cluster richness is an important characteristic that provides information on cluster mass (e.g., McNamara et al. 2001). Historically, cluster richness has been described using the Abell richness class (ARC), first defined by Abell (1958). Several studies have shown that the Abell richness parameter is not a well-

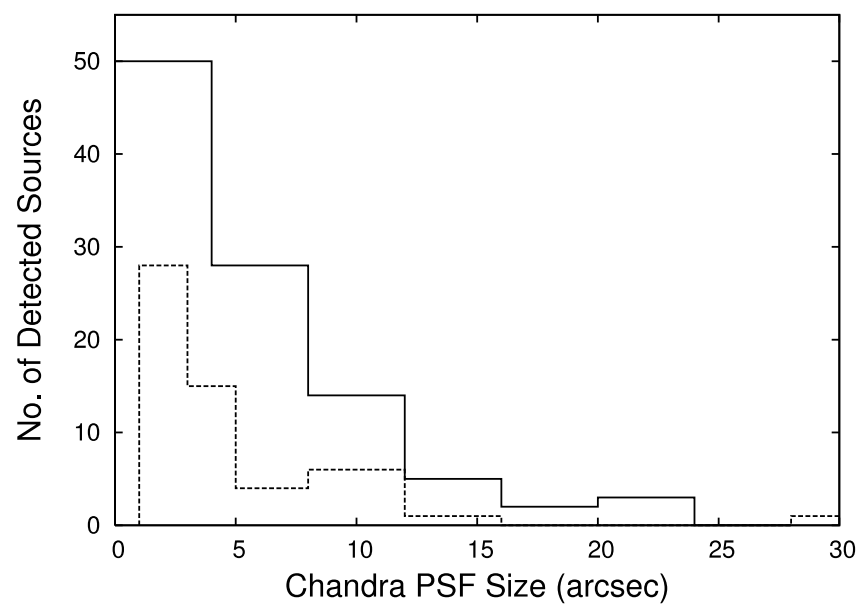

FIG. 19.-Comparison of the histogram distribution of detected candidate cluster sources as a function of the Chandra PSF size at the source position. The solid line depicts the distribution of the 102 optical VTP detections that were not detected as extended X-ray sources, while the dashed line represents the 55 extended X-ray sources. A K-S test demonstrates that the two distributions are not inconsistent with each other.

defined quantity and is subjected to numerous observational biases, including projection effects (e.g., Lucey 1983; van Haarlem et al. 1997; Miller et al. 1999). For our cluster sample, we have elected to use the parameter $B_{\mathrm{gc}}$ (Yee \& López-Cruz 1999; Yee \& Ellingson 2003) to characterize optical cluster richness. The $B_{\mathrm{gc}}$ parameter is a measure of the cluster center-galaxy correlation amplitude and is related to the correlation function defined by; $\xi(r)=B_{\mathrm{gc}} r^{-\gamma}$ (Longair \& Seldner 1979). Observationally, it is easier to compute the angular correlation function, which can be approximated using a power law of the form $w(\theta)=A_{\mathrm{gg}} \theta^{1-\gamma}$ (see Davis \& Peebles 1983) rather than $\xi(r)$. Determining the angular distribution of galaxies about the cluster center provides a measure of $A_{\mathrm{gg}}$, the number of background-subtracted galaxies within some angular radius $\theta$ of the adopted cluster center. The amplitude of the angular correlation function can be expressed as (assuming a fixed $\gamma) A_{\mathrm{gc}}=\left(N_{\text {net }} / N_{\mathrm{bg}}\right)[(3-\gamma) / 2] \theta^{\gamma-1}$, where $N_{\text {net }}$ is the background-corrected galaxy counts, and $N_{\mathrm{bg}}$ is the background counts (Yee \& López-Cruz 1999). Assuming spherical symmetry, $A_{\mathrm{gc}}$ and $B_{\mathrm{gc}}$ can be related (Longair \& Seldner 1979) via

$$
B_{\mathrm{gc}}=N_{\mathrm{bg}} \frac{D^{\gamma-3} A_{\mathrm{gc}}}{I_{\gamma} \Psi\left[M\left(m_{0}, z\right)\right]},
$$

where $N_{\text {bg }}$ is the background galaxy counts measured to apparent magnitude $m_{0}, D$ is the angular-diameter distance, $I_{\gamma}$ is an integration constant, and $\Psi\left[M\left(m_{0}, z\right)\right]$ is the integrated luminosity function to absolute magnitude $M\left(m_{0}, z\right)$ corresponding to the apparent magnitude limit $m_{0}$ at the cluster redshift.

The uncertainty of $B_{\mathrm{gc}}$ can be computed from (Yee \& LópezCruz 1999):

$$
\frac{\Delta B_{\mathrm{gc}}}{B_{\mathrm{gc}}}=\frac{\left(N_{\mathrm{net}}+1.3^{2} N_{\mathrm{bg}}\right)^{1 / 2}}{N_{\text {net }}},
$$

where the factor of $1.3^{2}$ accounts for the field-to-field fluctuation of background galaxy counts above the expected Poisson distribution (e.g., Yee \& Green 1987; López-Cruz 1997; Barkhouse 2003).

The $B_{\mathrm{gc}}$ parameter has been used in numerous studies to quantify the environment of quasars and radio galaxies (Yee \& Green 
TABLE 6

Published Sources near Optical-only Detections ${ }^{a}$

\begin{tabular}{|c|c|c|}
\hline No. & Published ID ${ }^{\mathrm{b}}$ & Comments \\
\hline 1 & 2MASX J00235911-0150171 & Galaxy: 0.0 from optical center \\
\hline 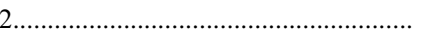 & 1WGA J0101.3+3146 & X-ray source within $60^{\prime \prime}$ of optical centroid \\
\hline 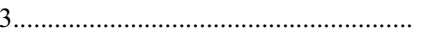 & $\ldots$ & $\ldots$ \\
\hline 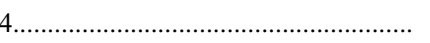 & NVSS J010156+314537 & Radio source within $24^{\prime \prime}$ of optical centroid \\
\hline 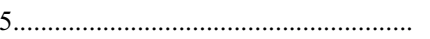 & $\ldots$ & $\ldots$ \\
\hline 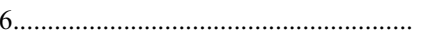 & 2MASX J01025380-2707225 & Galaxy $(z=0.221182): 0$."0 from optical center \\
\hline 7 & RX J0152.7-1357 & Galaxy: $54^{\prime \prime}$ from optical source \\
\hline 8 & CXOMP J015312.3-135723 & Galaxy/ChaMP X-ray point source: $54^{\prime \prime}$ from optical center \\
\hline ................. & CXOMP J015311.1-135104 & Galaxy/ChaMP X-ray point source: $114^{\prime \prime}$ from optical center \\
\hline 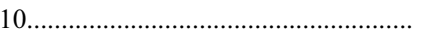 & $\ldots$ & $\ldots$ \\
\hline 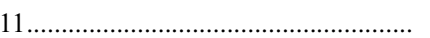 & APMUKS(BJ) B033414.59-050957.3 & Galaxy: $42^{\prime \prime}$ from optical source \\
\hline $12 \ldots \ldots \ldots \ldots \ldots \ldots \ldots$ & APMUKS(BJ) B033500.27-051957.8 & Galaxy: $42^{\prime \prime}$ from optical source \\
\hline $13 \ldots \ldots \ldots \ldots . . .$. & $\ldots$ & $\ldots$ \\
\hline 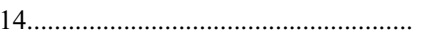 & $\ldots$ & $\ldots$ \\
\hline 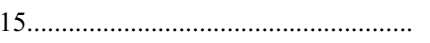 & $\ldots$ & $\ldots$ \\
\hline 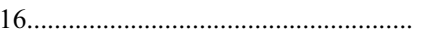 & $\ldots$ & $\ldots$ \\
\hline . & 2MASX J05422628-4049430 & Galaxy: $114^{\prime \prime}$ from optical source \\
\hline 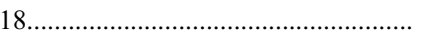 & $\ldots$ & $\ldots$ \\
\hline 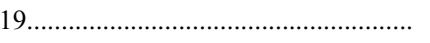 & $\cdots$ & $\cdots$ \\
\hline 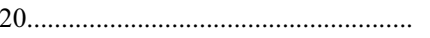 & $\ldots$ & $\ldots$ \\
\hline 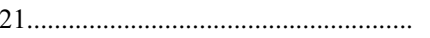 & $\ldots$ & $\ldots$ \\
\hline 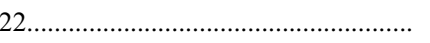 & $\ldots$ & $\ldots$ \\
\hline$\ldots \ldots \ldots \ldots \ldots \ldots$ & $\ldots$ & $\ldots$ \\
\hline 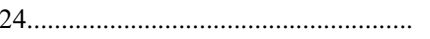 & $\ldots$ & $\ldots$ \\
\hline 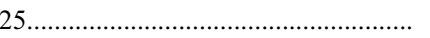 & LCRS B105416.2-032123 & Galaxy: $0 . " 0$ from optical centroid \\
\hline 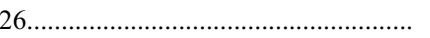 & SPS J105700.03-034400.9 & Galaxy: $18^{\prime \prime}$ from optical source position \\
\hline 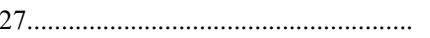 & $\ldots$ & $\ldots$ \\
\hline 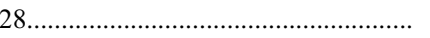 & NVSS J111718+075856 & Radio source: $24^{\prime \prime}$ of optical cluster \\
\hline $29 \ldots \ldots$. & MAPS-NGP O_553_0096538 & Galaxy: $96^{\prime \prime}$ from optical source \\
\hline 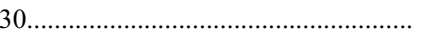 & MAPS-NGP O_553_0096538 & Galaxy: $66^{\prime \prime}$ from optical source \\
\hline 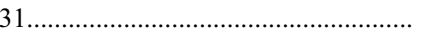 & SDSS J111816.59+074323.9 & Galaxy $(z=0.225281): 0.0$ from optical source \\
\hline 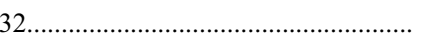 & NVSS J113953-263357 & Radio source: $48^{\prime \prime}$ from optical source \\
\hline 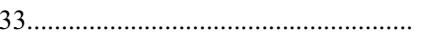 & $\ldots$ & $\ldots$ \\
\hline 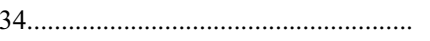 & $\ldots$ & $\ldots$ \\
\hline 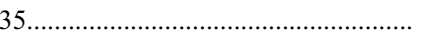 & PKS 1138-26: [PKC2002] 08 & $102^{\prime \prime}$ from optical source \\
\hline 36 & SDSS J125621.26+471555.4 & Galaxy $(z=0.208849): 0$."0 from optical source \\
\hline 37 & $\ldots$ & $\ldots$ \\
\hline 38 & CXOSEXSI J131637.2+290630 & X-ray source: $48^{\prime \prime}$ from optical cluster candidate \\
\hline 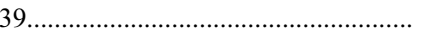 & CXOU J1316.9+2914 & Galaxy cluster: 0.0 from optical source \\
\hline 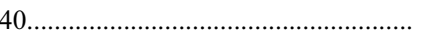 & CXOSEXSI J131714.0+292034 & X-ray source: $90^{\prime \prime}$ from optical centroid \\
\hline 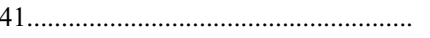 & CXOSEXSI J131721.8+285926 & X-ray source: $42^{\prime \prime}$ from optical source \\
\hline 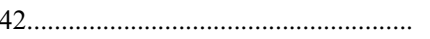 & NVSS J134719-115226 & Radio source within $12^{\prime \prime}$ of optical centroid \\
\hline 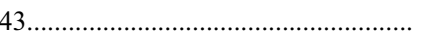 & 2MASX J13472770-1140397 & Galaxy $(z=0.086372): 00^{\prime \prime} 0$ from optical center \\
\hline 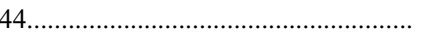 & OC03a J1415+2311 & Galaxy cluster: $48^{\prime \prime}$ from optical centroid $(z=0.500$ EST) \\
\hline 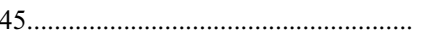 & 2MASX J14153929+2313477 & Galaxy: $78^{\prime \prime}$ from optical source \\
\hline 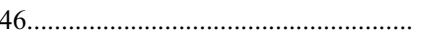 & MAPS-NGP O_382_0330729 & Galaxy: $90^{\prime \prime}$ from optical cluster candidate \\
\hline 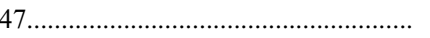 & MAPS-NGP O_500_0168801 & Galaxy: $54^{\prime \prime}$ from optical source \\
\hline 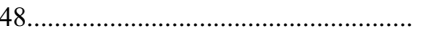 & [KSC90] 39 & Galaxy: $120^{\prime \prime}$ from optical source \\
\hline 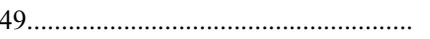 & NVSS J141601+112552 & Radio source within $42^{\prime \prime}$ of optical centroid \\
\hline .................... & SDSS J141609.07+444416.6 & Galaxy $(z=0.373182): 18^{\prime \prime}$ from optical centroid \\
\hline .................... & 2MASX J14162498+2304411 & Galaxy: $114^{\prime \prime}$ from optical cluster position \\
\hline .................. & 1WGA J1416.2+2321 & X-ray source: $114^{\prime \prime}$ from optical cluster position \\
\hline 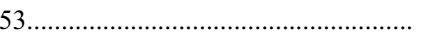 & CXOMP J141626.6+445240 & Galaxy/ChaMP X-ray point source: $12^{\prime \prime}$ from optical center \\
\hline 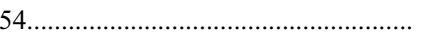 & $\ldots$ & $\ldots$ \\
\hline 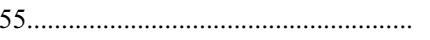 & $\ldots$ & $\ldots$ \\
\hline 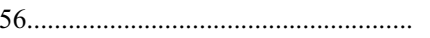 & NVSS J142337+235135 & Radio source within $114^{\prime \prime}$ of optical centroid \\
\hline 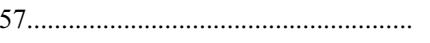 & $\cdots$ & $\ldots$ \\
\hline 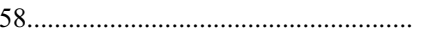 & $\ldots$ & $\ldots$ \\
\hline 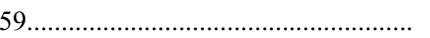 & $87 \mathrm{~GB} 142237.3+231854$ & Radio source within $108^{\prime \prime}$ of optical centroid \\
\hline 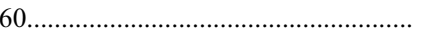 & NVSS J142507+225642 & Radio source within $48^{\prime \prime}$ of optical centroid \\
\hline 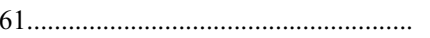 & SDSS J151346.73+364323.6 & Galaxy $(z=0.240430): 54^{\prime \prime}$ from optical centroid \\
\hline 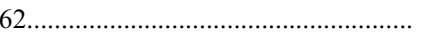 & MS 1512.4+3647:PPP 102417 & Galaxy $(z=403390): 12^{\prime \prime}$ from optical centroid \\
\hline 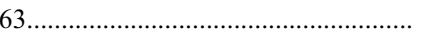 & 2MASX J15484523+2130178 & Galaxy: $108^{\prime \prime}$ from optical source \\
\hline 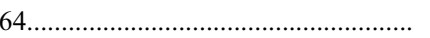 & NVSS J154901+213031 & Radio source: $18^{\prime \prime}$ from optical centroid \\
\hline 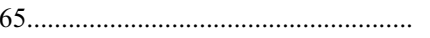 & OC02a J1549+2119 & Galaxy cluster: $66^{\prime \prime}$ from optical source $(z=0.200$ EST) \\
\hline 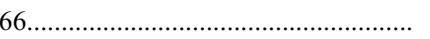 & OC03 J1549+2123 & Galaxy clusters: $12^{\prime \prime}$ from optical center $(z=0.700$ EST $)$ \\
\hline
\end{tabular}


TABLE 6-Continued

\begin{tabular}{|c|c|c|}
\hline No. & Published $\mathrm{ID}^{\mathrm{b}}$ & Comments \\
\hline $67 .$. & 3C $324 \mathrm{C} 006$ & Galaxy: $48^{\prime \prime}$ from optical source \\
\hline $68 \ldots \ldots \ldots \ldots \ldots \ldots \ldots \ldots$ & NVSS J160753+654404 & Radio source: $108^{\prime \prime}$ from optical cluster position \\
\hline .......................... & NVSS J160832+654437 & Radio source: $108^{\prime \prime}$ from optical cluster position \\
\hline $70 \ldots \ldots \ldots \ldots \ldots \ldots \ldots$ & 2MASX J16223328+2630454 & Galaxy: 0.0 from optical centroid \\
\hline ............................ & [KCW99] 28 & $102^{\prime \prime}$ from optical source \\
\hline 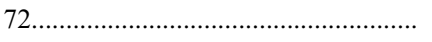 & 2MASX J17142140+5002487 & Galaxy: $36^{\prime \prime}$ from optical source \\
\hline 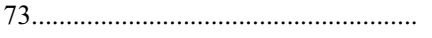 & $\ldots$ & $\ldots$ \\
\hline 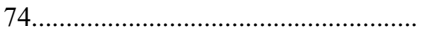 & $\ldots$ & $\ldots$ \\
\hline ................. & 2MASX J18072389+4546117 & Galaxy: $84^{\prime \prime}$ from optical source \\
\hline (n) & $\ldots$ & $\ldots$ \\
\hline ............................ & $\ldots$ & $\ldots$ \\
\hline $78 \ldots \ldots \ldots \ldots \ldots \ldots \ldots$ & CXOSEXSI J205537.3-043333 & X-ray source: $84^{\prime \prime}$ from optical source \\
\hline ............................ & CXOSEXSI J205649.1-044013 & X-ray source: $72^{\prime \prime}$ from optical centroid \\
\hline 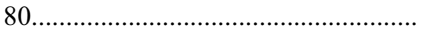 & CXOMP J213924.9-234221 & Galaxy/ChaMP X-ray point source $(z=0.401)$ : $36^{\prime \prime}$ from optical source \\
\hline 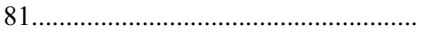 & $\ldots$ & $\ldots$ \\
\hline 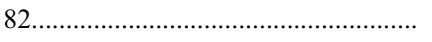 & APMUKS(BJ) B213729.27-235708.1 & Galaxy: $78^{\prime \prime}$ from optical centroid \\
\hline 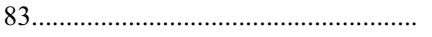 & $\ldots$ & $\ldots$ \\
\hline .......................... & LBQS 2154-2005 & QSO $(z=2.035000): 30^{\prime \prime}$ from optical source \\
\hline $85 \ldots$. & 2MASX J22045283-1815366 & Galaxy: 0.0 from optical centroid \\
\hline $86 \ldots . .$. & {$[\mathrm{B} 2002 \mathrm{a}] 35$} & Galaxy cluster: 0.0 from optical source \\
\hline $87 \ldots . .$. & APMUKS(BJ) B221007.01-222620.9 & $54^{\prime \prime}$ from optical centroid \\
\hline 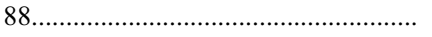 & APMUKS(BJ) B221035.05-223249.1 & $78^{\prime \prime}$ from optical cluster \\
\hline (n)...................... & ANTI-LEONID: [CME2001] 24 & X-ray source: $12^{\prime \prime}$ from optical cluster position \\
\hline (n...................... & APMUKS(BJ) B221513.16+000716.2 & Galaxy: $24^{\prime \prime}$ from optical source \\
\hline 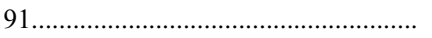 & CFRS 22.0770 & Galaxy $(z=0.818800): 12^{\prime \prime}$ from optical source \\
\hline 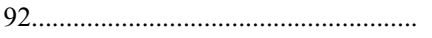 & SDSS J221833.05+001737.3 & Galaxy $(z=0.332172): 0.0$ from optical source \\
\hline …............... & NVSS J223948+033801 & Radio source: $102^{\prime \prime}$ from optical centroid \\
\hline (............... & 2MASX J22395403+0332457 & Galaxy: $78^{\prime \prime}$ from optical centroid \\
\hline .................. & 2MASX J22402529+0333046 & Galaxy: $108^{\prime \prime}$ from optical centroid \\
\hline 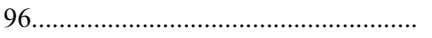 & 2MASX J22402529+0333046 & Galaxy: $54^{\prime \prime}$ from optical centroid \\
\hline (n)....................... & {$[\mathrm{B} 2002 \mathrm{a}] 36$} & Galaxy cluster: $102^{\prime \prime}$ from optical cluster position \\
\hline (n......................... & NVSS J230312+085012 & Radio source: $66^{\prime \prime}$ from optical position \\
\hline .............................. & CXOSEXSI J234758.8+010344 & X-ray source: $6^{\prime \prime}$ from optical centroid \\
\hline 100 & CXOSEXSI J234815.8+005351 & X-ray source: $6^{\prime \prime}$ from optical centroid \\
\hline 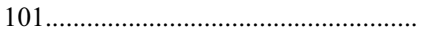 & CXOSEXSI J234839.5+010828 & X-ray source: $12^{\prime \prime}$ from optical position \\
\hline 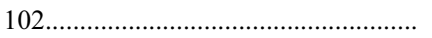 & APMUKS(BJ) B234658.77+004331.4 & Galaxy: $54^{\prime \prime}$ from optical cluster \\
\hline
\end{tabular}

a Published references acquired from NED.

$\mathrm{b}$ Blank field if published sources $>2^{\prime}$ from object position.

1984, 1987; Prestage \& Peacock 1988; Yates et al. 1989; Ellingson et al. 1991), BL Lacertae objects (Smith et al. 1995; Wurtz et al. 1997), Seyfert galaxies (De Robertis et al. 1998), Abell clusters (Anderson \& Owen 1994; López-Cruz 1997; Yee \& López-Cruz 1999; Barkhouse 2003), ROSAT clusters (Gilbank et al. 2004), and Red-Sequence Cluster Survey (RCS) clusters (Gladders \& Yee 2000; Hicks et al. 2005).

The primary steps associated with measuring $B_{\mathrm{gc}}$ for our cluster sample involve counting galaxies to a fixed absolute magnitude within a fixed physical radius of the adopted cluster center. The galaxy counts are then background-corrected using the luminosity function generated from four randomly positioned deep ChaMP optical images that do not contain known clusters or bright stars. Galaxies are counted within a radius of $0.5 h_{70}^{-1} \mathrm{Mpc}$ from the cluster center. The cluster center is defined as the centroid of the brightest cluster galaxy or the center of the galaxy density enhancement if no obvious BCG exists. Following the procedure of Yee \& López-Cruz (1999), we include only those galaxies brighter than the $K$ - and evolution-corrected value $(Q=-1.4 z$; Yee \& López-Cruz 1999) of $M^{*}+2$, where $M^{*}$ is the value of $M_{i^{\prime}}^{*}=-21.52$ as implemented for our red-sequence-filtered VTP technique (see $\S 3.2 .1$ ). In addition to the general procedure outlined in Yee \& López-Cruz (1999), we only include galaxies that have $r^{\prime}-i^{\prime}$ colors within \pm 0.5 mag of the cluster red sequence for our specific target (identical color cuts were also applied to the background galaxy population). This colorselection step helps to minimize uncertainties in the luminosity function and galaxy evolutionary corrections at $z \sim 0.4$ (the median redshift of our sample) by statistically selecting redsequence early-type galaxies with known properties (see Hicks et al. 2005 for a similar application).

In Figure 20 we plot $L_{\mathrm{X}}$ versus $B_{\mathrm{gc}}$ for three cluster samples: (1) the cluster sample with X-ray and optical matches (filled circles), (2) the optical-only clusters detected by VTP (arrows), and (3) a sample of 35 Abell clusters (filled triangles) from Barkhouse (2003). We include the Abell cluster sample to provide a reference of the expected range of richness of known clusters. The X-ray luminosity values for the Abell cluster sample are taken from Ledlow et al. (2003) for the 0.5-2 keV energy band and transformed to our cosmology. It is apparent from the figure that $B_{\mathrm{gc}}$ is weakly correlated with $L_{\mathrm{X}}$ such that richer clusters (as denoted by larger values of $B_{\mathrm{gc}}$ ) are more luminous in the X-rays. The large scatter between these measurements has also been seen in previous studies (Yee \& Ellingson 2003; Gilbank et al. 2004; Hicks et al. 2005). Due to the relatively bright optical magnitude limits compared to $m_{i^{\prime}}^{*}$ for three fields, one cluster from the 


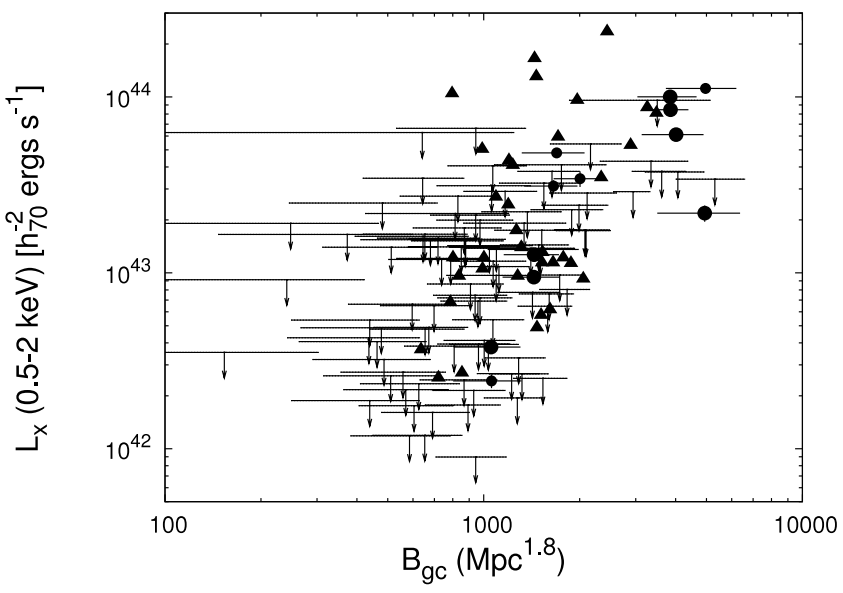

FIG. 20.-X-ray luminosity $(0.5-2 \mathrm{keV})$ vs. the richness parameter $B_{\mathrm{gc}}$ for three cluster samples. The matched X-ray/optical cluster sample is depicted with the filled circles, while the optical-only clusters are represented by the arrows (upper limits for $L_{\mathrm{X}}$ ). Clusters with spectroscopic redshifts are plotted with larger symbol size, while sources with VTP redshifts are shown with the smaller symbols. For comparison purposes, a sample of 35 Abell clusters is shown by the filled triangles. All uncertainties are $1 \sigma$ values.

X-ray/optically matched sample and eight from the optical-only group are not included in the $B_{\mathrm{gc}}$ analysis since galaxies could not be counted to the adopted absolute magnitude limit.

The $B_{\mathrm{gc}}$ values for all three groups of clusters have been measured in a consistent manner using the same cosmology and selection of galaxies relative to the individual cluster red sequence. The median value of $B_{\mathrm{gc}}$ for the three samples are $B_{\mathrm{gc}}^{\mathrm{med}}=$ $1849 \pm 236$ for the group of clusters with X-ray and optical counterparts, $B_{\mathrm{gc}}^{\mathrm{med}}=944 \pm 47$ for the optical-only detected clusters, and $B_{\mathrm{gc}}^{\mathrm{med}} \stackrel{\mathrm{gc}}{=} 1444 \pm 114$ for the 35 Abell clusters.

The median richness of the optical-only clusters is $3.8 \sigma$ lower than the value measured for the matched X-ray/optical sample. This provides additional support for the conclusions drawn from Figure 9 and 10, that the optical-only cluster sample consists mainly of poor clusters and groups that are too X-ray weak to be detected in our sample. However, there is evidence in Figure 20 that some of the optical sources not detected in the X-ray have optical richness consistent with Abell-like clusters (see $\S 5.0$ ).

\section{DISCUSSION AND CONCLUSIONS}

The primary goal of this paper is to present the X-ray and optical properties of a compilation of extended X-ray sources discovered serendipitously as part of ChaMP. The availability of $\sim 13 \mathrm{deg}^{2}$ of Chandra archival data with deep mosaic optical coverage from 56 NOAO 4 m fields allows us to test independently cluster detection schemes in the X-ray and optical passbands. By cross-correlating the resulting compilations from the overlapping $6.1 \mathrm{deg}^{2}$, we are able to extend the X-ray and optical analysis of clusters/groups to lower X-ray luminosities than previous ROSAT cluster surveys such as REFLEX (see Fig. 10). The 160 Square Degree ROSAT Survey provides a better match to our sample than the REFLEX survey. The 160 Square Degree survey covers the same range in redshift and $L_{\mathrm{X}}$ as the ChaMP cluster survey but includes a larger fraction of sources at lower redshift.

From our sample of 55 extended X-ray sources, we measured a matched fraction of $46 \%$ (13 out of 28$)$ with cluster candidates detected by our red-sequence-filtered VTP method. This matched fraction only includes those sources that are located in areas that have overlapping X-ray and optical coverage, and excludes the six $\mathrm{X}$-ray sources that are coincident with single bright galaxies in the optical. The $46 \%$ matched fraction is not too different from typical values measured in other X-ray/optical studies. Donahue et al. (2002) found that $60 \%$ (26 out of 43) of their ROSAT-detected clusters have optical counterparts as measured by their matchedfilter algorithm. This is similar to Kolokotronis et al. (2006) who matched $68 \%$ (13 out of 19) of their XMM-Newton sources with optical clusters detected using a smoothing percolation method. We note, however, that a direct comparison to other studies is problematic given that the matched fraction is expected to depend on the flux limit achieved in each passband and the X-ray-tooptical flux ratio for specific types of galaxy clusters.

Since there is no such thing as the "perfect" cluster detector, each cluster-finding method is subject to bias. The X-ray wavelet decomposition technique relies on the ability to separate extended and point sources. This task becomes more difficult the larger the off-axis angle and can prevent the inclusion of extended X-ray sources that are found near the field edge. In addition, poor clusters or groups with shallow potential wells may only emit detectable $\mathrm{X}$-rays from regions smaller than the Chandra PSF. The redsequence-filtered VTP optical cluster detection technique relies on the presence of the early-type galaxy red sequence to help improve the contrast of clusters above the field galaxy population. For increasing redshift, the fraction of blue cluster galaxies (usually associated with later types) has been found to increase (the well known Butcher-Oemler effect; Butcher \& Oemler 1984). The increase in the blue fraction will have an effect on the efficiency of any optical cluster finder that relies on the existence of the cluster red sequence (e.g., Gladders \& Yee 2000; Donahue et al. 2002).

A comparison of the sample of optically selected VTP clusters with those detected in the X-rays yields a matched fraction of $11 \%$ (i.e., only 13 out of 115 optical clusters have a detected X-ray counterpart). As discussed in $\S 4.3$, many of these sources have X-ray upper limits that are consistent for a population of groups and "normal" clusters (see Fig. 9). A comparison of the richness of the optical-only versus X-ray/optically matched cluster samples (see Fig. 20) shows that the average richness of the optical-only VTP sample is smaller than the cluster sample with $\mathrm{X}$-ray and optical counterparts by $\sim 4 \sigma$. This result supports the hypothesis that the optical-only sample is either (1) composed mainly of poor systems that are undetected by our X-ray observations due to the lack of sufficient hot intracluster gas or (2) contaminated by nonvirialized filaments of the large-scale structure. In addition, the comparison of optical richness as characterized by $B_{\mathrm{gc}}$ (see Fig. 20) shows the presence of several clusters not detected in our X-ray data that have an optical richness similar to Abell-type clusters. We examined the possible impact that the $\mathrm{X}$-ray exposure time has on the detection of these systems by looking at the median exposure times for the VTP-only detected sources with $B_{\mathrm{gc}}>3000$ (five sources) and those X-ray-detected sources with $B_{\mathrm{gc}}>3000$ (also five sources). The median vignettingcorrected X-ray exposure time of the VTP-only sources is $19.5 \pm$ $12.7 \mathrm{ks}$, where the uncertainty is the dispersion. For the X-raydetected sources we find a median value of $44.9 \pm 12.4 \mathrm{ks}$. The median X-ray exposure times of the positions of the VTP-only detected clusters is at most $1.4 \sigma$ lower than the X-ray-detected extended sources. Including clusters of lower $B_{\mathrm{gc}}$ further decreases the significance of this difference $\left(0.5 \sigma\right.$ for $\left.B_{\mathrm{gc}}>2000\right)$. This analysis indicates that it is unlikely that many of the VTP-only detected optical sources are missed in the X-rays due to shallow $\mathrm{X}$-ray exposure times.

To increase the cluster sample size and search for rare luminous X-ray clusters, we will extend this study to include archival images from Chandra Cycles 3-6. We will use primarily SDSS 
photometry for optical coverage, and a red-sequence VTP method to discriminate cluster red sequences out to $z \sim 1.1$, or 0.5 if we only include galaxies brighter than $m_{z^{\prime}}^{*}$ (based on the optical magnitude limit of the SDSS $z^{\prime}$-band data; i.e., $z_{\lim }^{\prime} \sim 20.5$ ).

We gratefully acknowledge support for this project through NASA under CXC archival research grant AR4-5017X. R. A. C.,
P. J. G., D. W. K., A. E. M., H. T., and B. J. W. also acknowledge support through NASA contract NAS8-39073 (CXC). The authors thank the staff at CTIO, KPNO, and Las Campanas Observatory for assistance with the optical observations presented here. We thank Taddy Kodama for use of his cluster redsequence models and Howard Yee for discussions and access to his $B_{\mathrm{gc}}$ code. We also thank the referee for helpful comments and suggestions.
Abell, G. O. 1958, ApJS, 3, 211

Akritas, M. G., \& Siebert, J. 1996, MNRAS, 278, 919

Anderson, V., \& Owen, F. 1994, AJ, 108, 361

Bahcall, N. A., et al. 2003, ApJS, 148, 243

Barkhouse, W. A. 2003, Ph.D. thesis, Univ. Toronto

Barnes, J. E. 1989, Nature, 338, 123

Basilakos, S., Plionis, M., Georgakakis, A., Georgantopoulos, I., Gaga, T., Kolokotronis, V., \& Stewart, G. C. 2004, MNRAS, 351, 989

Baum, W. A. 1959, PASP, 71, 106

Bennett, C. L., et al. 2003, ApJS, 148, 1

Bertin, E., \& Arnouts, S. 1996, A\&AS, 117, 393

Böhringer, H., et al. 2004, A\&A, 425, 367

Bower, R. G., Lucey, J. R., \& Ellis, R. S. 1992, MNRAS, 254, 601

Bruzual, G., \& Charlot, S. 2003, MNRAS, 344, 1000

Butcher, H., \& Oemler, A., Jr. 1984, ApJ, 285, 426

Cavaliere, A., \& Fusco-Femiano, R. 1976, A\&A, 49, 137

Davis, M., \& Peebles, P. J. E. 1983, ApJ, 267, 465

De Robertis, M. M., Yee, H. K. C., \& Hayhoe, K. 1998, ApJ, 496, 93

Donahue, M., et al. 2002, ApJ, 569, 689

Dubinski, J. 1998, ApJ, 502, 141

Ebeling, H., \& Wiedenmann, G. 1993, Phys. Rev. E, 47, 704

Ellingson, E., Yee, H. K. C., \& Green, R. F. 1991, ApJ, 371, 49

Ellis, R. S., Smail, I., Dressler, A., Couch, W. J., Oemler, A., Butcher, H., \& Sharples, R. M. 1997, ApJ, 483, 582

Ettori, S., Tozzi, P., Borgani, S., \& Rosati, P. 2004, A\&A, 417, 13

Fabbiano, G. 1989, ARA\&A, 27, 87

Frei, Z., \& Gunn, J. E. 1994, AJ, 108, 1476

Fukugita, M., Ichikawa, T., Gunn, J. E., Doi, M., Shimasaku, K., \& Schneider, D. P. 1996, AJ, 111, 1748

Gilbank, D. G., Bower, R. G., Castander, F. J., \& Ziegler, B. L. 2004, MNRAS, 348,551

Gladders, M. D., \& Yee, H. K. C. 2000, AJ, 120, 2148

Gonzalez, A. H., Zaritsky, D., Dalcanton, J. J., \& Nelson, A. 2001, ApJS, 137, 117

Goto, T., et al. 2002, AJ, 123, 1807

Green, P. J., et al. 2004, ApJS, 150, 43

Hicks, A. K., Ellingson, E., Bautz, M., Yee, H. K. C., Gladders, M., \& Garmire, G. 2005, Adv. Space Res., 36, 706

Hsieh, B. C., Yee, H. K. C., Lin, H., \& Gladders, M. D. 2005, ApJS, 158, 161

Humason, M. L., Mayall, N. U., \& Sandage, A. R. 1956, AJ, 61, 97

Jones, C., \& Forman, W. 1999, ApJ, 511, 65

Jones, L. R., Ponman, T. J., Horton, A., Babul, A., Ebeling, H., \& Burke, D. J. 2000, MNRAS, 312, 139

Kiang, T. 1966, Z. Astrophys., 64, 433

Kim, D.-W., et al. 2004a, ApJS, 150, 19 $2004 \mathrm{~b}, \mathrm{ApJ}, 600,59$ 2006, ApJ, 644, 829

Kim, R. S. J., et al. 2002, AJ, 123, 20

Kodama, T., \& Arimoto, N. 1997, A\&A, 320, 41

Kolokotronis, V., Georgakakis, A., Basilakos, S., Georgantopoulos, I., Plionis, M., Kitsionas, S., \& Gaga, T. 2006, MNRAS, 366, 163

Landolt, A. U. 1992, AJ, 104, 372

Ledlow, M. J., Voges, W., Owen, F. N., \& Burns, J. O. 2003, AJ, 126, 2740

Longair, M. S., \& Seldner, M. 1979, MNRAS, 189, 433

López-Cruz, O. 1997, Ph.D. thesis, Univ. Toronto

López-Cruz, O., Barkhouse, W. A., \& Yee, H. K. C. 2004, ApJ, 614, 679

\section{REFERENCES}

Lucey, J. R. 1983, MNRAS, 204, 33

Mamon, G. A. 1987, ApJ, 321, 622

McNamara, B. R., Vikhlinin, A., Hornstrup, A., Quintana, H., Whitman, K., Forman, W., \& Jones, C. 2001, ApJ, 558, 590

Miller, C. J., Batuski, D. J., Slinglend, K. A., \& Hill, J. M. 1999, ApJ, 523, 492

Mohr, J. J., Reese, E. D., Ellingson, E., Lewis, A. D., \& Evrard, A. E. 2000, ApJ, 544, 109

Moore, B., Lake, G., Stadel, J., \& Quinn, T. 1999, in ASP Conf. Ser. 170, Low Surface Brightness Universe, ed. J. I. Davies, C. Impey, \& S. Phillipps (San Francisco: ASP), 229

Moretti, A., et al. 2004, A\&A, 428, 21

Mulchaey, J. S. 2000, ARA\&A, 38, 289

Mullis, C. R., et al. 2003, ApJ, 594, 154

Nichol, R. C. 2004, in Clusters of Galaxies: Probes of Cosmological Structure and Galaxy Formation, ed. J. S. Mulchaey, A. Dressler, \& A. Oemler (Cambridge: Cambridge Univ. Press), 24

Oke, J. B., \& Sandage, A. 1968, ApJ, 154, 21

Ponman, T. J., Allen, D. J., Jones, L. R., Merrifield, M., McHardy, I. M., Lehto, H. J., \& Luppino, G. A. 1994, Nature, 369, 462

Popesso, P., Böhringer, H., Brinkmann, J., Voges, W., \& York, D. G. 2004, A\&A, 423, 449

Postman, M. 2002, in ASP Conf. Proc. 268, Tracing Cosmic Evolution with Galaxy Clusters, ed. S. Borgani, M. Mezzetti, \& R. Valdarnini (San Francisco: ASP), 3

Postman, M., Lubin, L. M., Gunn, J. E., Oke, J. B., Hoessel, J. G., Schneider, D. P., \& Christensen, J. A. 1996, AJ, 111, 615

Press, W. H., \& Schechter, P. 1974, ApJ, 187, 425

Prestage, R. M., \& Peacock, J. A. 1988, MNRAS, 230, 131

Ramella, M., Boschin, W., Fadda, D., \& Nonino, M. 2001, A\&A, 368, 776

Rosati, P., Borgani, S., \& Norman, C. 2002, ARA\&A, 40, 539

Sandage, A., \& Visvanathan, N. 1978, ApJ, 225, 742

Schechter, P. 1976, ApJ, 203, 297

Schlegel, D. J., Finkbeiner, D. P., \& Davis, M. 1998, ApJ, 500, 525

Silverman, J. D. 2004, Ph.D. thesis, Univ. Virginia

Silverman, J. D., et al. 2005a, ApJ, 618, 123 2005b, ApJ, 624, 630

Smith, E. P., O’Dea, C. D., \& Baum, S. 1995, ApJ, 441, 113

Stark, A. A., Gammie, C. F., Wilson, R. W., Bally, J., Linke, R. A., Heiles, C., \& Hurwitz, M. 1992, ApJS, 79, 77

Ulmer, M. P., et al. 2005, ApJ, 624, 124

van Haarlem, M. P., Frenk, C. S., \& White, S. D. M. 1997, MNRAS, 287, 817

Vikhlinin, A., McNamara, B. R., Forman, W., Jones, C., Quintana, H., \& Hornstrup, A. 1998a, ApJ, 498, L21 1998b, ApJ, 502, 558

Vikhlinin, A., McNamara, B. R., Hornstrup, A., Quintana, H., Forman, W., Jones, C., \& Way, M. 1999, ApJ, 520, L1

White, D. A., Jones, C., \& Forman, W. 1997, MNRAS, 292, 419

Wurtz, R., Stocke, J. T., Ellingson, E., \& Yee, H. K. C. 1997, ApJ, 480, 547

Yates, M. G., Miller, L., \& Peacock, J. A. 1989, MNRAS, 240, 129

Yee, H. K. C., \& Ellingson, E. 2003, ApJ, 585, 215

Yee, H. K. C., \& Green, R. F. 1984, ApJ, 280, 79 1987, ApJ, 319, 28

Yee, H. K. C., \& López-Cruz, O. 1999, AJ, 117, 1985

Zwicky, F., Herzog, E., \& Wild, P. 1961, Catalogue of Galaxies and Clusters of Galaxies, Vol. 1 (Pasadena: Caltech) 